\title{
A Stochastic Multi-Scale Approach for the Modeling of Thermo-Elastic Damping in Micro-Resonators
}

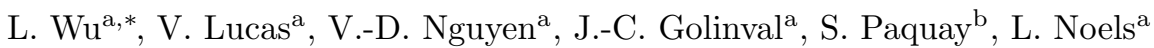 \\ ${ }^{a}$ University of Liege, Department of Aeronautics and Mechanical Engineering \\ Allée de la découverte 9, B-4000 Liège, Belgium \\ ${ }^{b}$ Open-Engineering SA, Rue Bois Saint-Jean 15/1 4102 Seraing, Belgium
}

\begin{abstract}
The aim of this work is to study the thermo-elastic quality factor (Q) of micro-resonators with a stochastic multi-scale approach. In the design of high-Q micro-resonators, thermo-elastic damping is one of the major dissipation mechanisms, which may have detrimental effects on the quality factor, and has to be predicted accurately. Since material uncertainties are inherent to and unavoidable in micro-electromechanical systems (MEMS), the effects of those variations have to be considered in the modeling in order to ensure the required MEMS performance. To this end, a coupled thermo-mechanical stochastic multi-scale approach is developed in this paper. Thermo-mechanical micro-models of polycrystalline materials are used to represent micro-structure realizations. A computational homogenization procedure is then applied on these statistical volume elements to obtain the stochastic characterizations of the elasticity tensor, thermal expansion, and conductivity tensors at the meso-scale. Spatially correlated meso-scale random fields can thus be generated to represent the stochastic behavior of the homogenized material properties. Finally, the distribution of the thermo-elastic quality factor of MEMS resonators is studied through a stochastic finite element method using as input the generated stochastic random field.
\end{abstract}

Keywords: Thermo-elasticity, Quality factor, Stochastic Multi-scale, MEMS, polycrystalline

\section{Introduction}

In the micro-electromechanical systems (MEMS) community, there are increasing demands in developing reliable micro-structures with very high quality factors $(\mathrm{Q})$. These micro-structures constitute the essential active part of applications such as resonant sensors and RF-MEMS filters, for which increasing the sensitivity and resolution of the devices is a critical issue. In order to obtain high-Q micro-resonators, all dissipation mechanisms that contribute to decreasing the quality factor have to be identified and well considered at the

\footnotetext{
* Corresponding author, Phone: +32436694 53, Fax: +3243669505

Email addresses: L.Wu@ulg.ac.be (L. Wu), Vincent.Lucas@ulg.ac.be (V. Lucas), vandung.nguyen@ulg.ac.be (V.-D. Nguyen), JC.Golinval@ulg.ac.be (J.-C. Golinval), s.paquay@open-engineering.com (S. Paquay), L.Noels@ulg.ac.be (L. Noels) 
design stage. The energy dissipation mechanisms of micro-resonators can be classified into two categories [46]. On the one hand, the majority of dissipation mechanisms are extrinsic, which means that they can be minimized by a proper design and operating conditions, such as by minimizing the air damping effect. Intrinsic losses, on the other hand, cannot be controlled as easily as extrinsic ones. Thermo-elastic damping has been identified as one kind of important intrinsic loss in high-Q micro-resonators $[8,15]$.

Thermo-elastic damping is an intrinsic energy dissipation which occurs through heat conduction. In a thermo-elastic solid, the thermal and mechanical fields have a strong coupling through the thermal expansion effect. MEMS resonators generally contain elements which vibrate in flexural modes and can be approximate by beams. In a vibrating beam, the two opposite sides undergo opposite deformations. When one side is compressed and its temperature increases consequently, the other side is stretched with the decrease of temperature. Thus, temperature gradients are generated and an energy dissipation occurs. However, this dissipation has a measurable influence only when the vibration frequency is of the order of the thermal relaxation rate. On the one hand, when the vibration frequency is much lower than the thermal relaxation rate, the vibrations are isothermal since the solid is always in thermal equilibrium. On the other hand, when the vibration frequency is much larger than the thermal relaxation rate, the vibrations are adiabatic since the system has no time for thermal relaxation. In MEMS, due to the small dimensions, the relaxation times of both the mechanical and thermal fields have similar order of magnitude and hence, thermo-elastic damping becomes important. Therefore, accurate modeling and prediction of energy loss due to the thermo-elastic effects become key requirements in order to improve the performance of high-Q resonators.

The early studies of thermo-elastic damping were mainly based on analytical models, which were derived for very simple structures and are subject to very restrictive assumptions. Zener [48] has developed a so-called Zener's standard model to approximate thermo-elastic damping for flexural vibrations of thin rectangular beams. Based on an extension of Hooke's law to the "Standard Anelastic Solid", which involves the stress $\sigma$, strain $\varepsilon$ as well as their first time derivatives $\dot{\sigma}, \dot{\varepsilon}$, the vibration characteristics of the solid is analyzed with the harmonic stress and strain. However Zener's theory does not provide the estimation of the frequency shift induced by thermo-elastic effects. For this purpose, Lifshitz and Roukes (LR) [21] have developed the thermo-elastic equations of a vibrating beam based on the same fundamental physics than Zener, which model more accurately the transverse temperature profile. The analytical models can be used to obtain the complex thermo-elastic resonant pulsation $\varpi_{n}$ and its corresponding quality factor $Q$ for simplified cases only. The limitation of analytical models and the complexity of the real micro-structures (i.e. non rectangular geometry, complex 3-D structures, anisotropic material,...) have motivated the development of numerical models [20,35], and the application of the finite element method to study the thermo-elastic damping has been validated by the comparisons of numerical results with analytical results.

However, deterministic finite element models are not accurate enough to obtain a reliable analysis of the performance of micro-resonators, as stressed in [20]. Indeed, MEMS are subject to inevitable and 
inherent uncertainties in their dimensional parameters and material properties which lead to variability in their performance and reliability. Due to the small dimensions of MEMS, manufacturing processes leave substantial variability in the shape and geometry of the device, while the material properties of a component are inherently subject to scattering. The effects of these variations have to be considered and a stochastic modeling methodology is needed in order to ensure the required MEMS performance under uncertainties.

Along this line, the perturbation Stochastic Finite Element Method (SFEM) was used in [20] to predict the variance of the thermo-elastic quality factor by considering isotropic and homogeneous material properties -Young's modulus, conductivity, and density- which uncertainties were represented through assumed variances. A pseudo-geometric variable was adopted to consider the geometric uncertainty. However, because of the reduced size of MEMS, the structural material appears anisotropic and non-homogeneous at this scale. The physically sensible description of the uncertainties should thus be obtained from the study of the heterogeneous nature of the material, while the rational modeling of geometric uncertainty should rely on the knowledge of the real manufacturing processes.

To account for the material heterogeneities, a direct finite element analysis, also called Monté-Carlo Simulation (MCS) method, can be used to analyze the structural response variability. This method was adopted in the fracture analysis of MEMS under shock in [26]. However the MCS method can lead to an overwhelming computation cost as it involves the finite-element discretization of all the heterogeneities. If the local response, as a crack initiation [26] is sought, such an approach is required, but if the concerned performance is a global response, such a level of discretization should be avoided. To circumvent the need for the discretization of the sub-microscopic structure, the uncertainty due to the material heterogeneities can be described as the spatial variability of the material properties in the structure through a random field. In the context of stochastic finite element analyzes, this random field is discretized in accordance with the structure finite element mesh; therefore finite element sizes smaller than the correlation length are required to ensure the accuracy of the analysis results as shown in [11]. In all generalities, the correlation length of the random material property depends on the characteristic length of the material heterogeneity, such as the size of the grains for polycrystalline materials. Thus the rather small correlation length results in a very expensive structural analyses in terms of computational resources. Besides the issue related to the computational cost, a finite element model based on the explicit micro-heterogeneities discretization leads to a noise field [3] instead of a smooth one [27], which prevents the use of a stochastic finite element method, such as the Neumann expansion [44] or the perturbation approximation [36]. Therefore, in order to solve the problem of structural stochasticity of heterogeneous materials, such as polycrystalline materials, at a reasonable computation cost, a stochastic multi-scale approach is required as discussed in [22].

The interest in multi-scale methods was renewed with the rise of structural applications made of heterogeneous materials such as composite materials, metal alloys, polycrystalline materials... As a particular case of multi-scale methods, homogenization methods relate the macroscopic strain tensor to the macroscopic 
stress tensor through the resolution of a meso-scale Boundary Value Problem (BVP) defined on a Representative Volume Elements (RVE), which represents the micro-structure and the micro-structural behavior in a statistically representative way. However, for a multi-scale analysis to be accurate, the scales separation criteria should be satisfied. Firstly, the size of the meso-scale volume element on which the homogenization is applied should be smaller than the characteristic length on which the macro-scale loading varies in space [9]. This criterion should always be satisfied. Secondly, to be statistically representative and thus to qualify to be a RVE, the meso-scale volume element should be much larger than the micro-structural size [12]. The size of the meso-scale volume element leading to statistical representativity can be determined based on statistical considerations of the homogenized properties [16, 13].

However, when the structural-size is not several orders of magnitude larger than the micro-structural size, the second criterion cannot be satisfied while respecting the first one, and the meso-scale volume element size should be reduced, leading to Statistical Volume Element (SVE) as discussed in [32]. Since an SVE is not statistically representative by definition, the homogenized meso-scale responses change with the SVE size, with the applied Boundary Conditions (BCs) on the SVE, but also for different SVE realizations of the same size. This last property of SVEs has been used to up-scale the uncertainties at the micro-scale to the meso-scale, for example to define the probability convergence criterion of RVE for masonry [10], to study the scale-dependency of homogenization for random composite materials [42], to obtain the property variations of poly-silicon film [25], to extract effective properties of random two-phase composites [39], or again to capture the stochastic properties of the parameters in a constitutive model [47]. The problem of finite elasticity was also considered through the resolution of composite material elementary cells in [4, 24], which allows defining a meso-scale potential as proposed in [4].

In [22], the authors, have applied the computational homogenization on a spatial sequence of SVEs to extract spatially correlated meso-scale statistical properties. A meso-scale random field of the homogenized material properties could thus be generated to feed the stochastic finite element method performed at the structural scale. As a result, the statistical distribution of the MEMS properties, for example the analysis of the micro-beams eigen-frequency, could be extracted. In this work, it was shown that the definition of a spatially correlated meso-scale random field is the key ingredient of the stochastic multi-scale approach; in order for the stochastic finite element method to converge, the size of the elements at the structural scale should remain lower than the spatial correlation length of the meso-scale random field, which in turn depends on the SVE size. This approach was also applied in [23] to study the effect of the geometric uncertainties resulting from the surface roughness, together with material uncertainties, on the vibration of micro-beams. To this end, a stochastic second-order homogenization process was coupled with structural shell stochastic finite elements to take the variation of surface geometry into account.

In these works, only the elastic material properties were considered. However, as the thermo-elastic damping results from the coupling of the thermal and mechanical processes, the extraction of the homoge- 
nized thermo-mechanical properties of the heterogeneous materials is required in the analysis of the quality factor of MEMS. In this paper, we extend the stochastic multi-scale methodology presented in [22] to account for the material uncertainties in a fully coupled thermo-mechanical homogenization process. As a result, a meso-scale random field of the material properties, which include the elasticity tensors, conductivity tensors, and thermal expansion tensors, can be generated to perform multi-physics stochastic finite element simulations in order to extract the variability of the thermo-elastic quality factor for micro-resonators.

The paper is organized as follows. In Section 2, after having recalled the governing equations of thermoelasticity coupling, (macro-scale) thermo-mechanical stochastic finite elements are described in order to study the thermo-elastic damping effect in a probabilistic way. In order to define the random fields of the meso-scale thermo-mechanical properties required by the stochastic finite elements, a thermo-mechanical stochastic homogenization scheme is first developed in Section 3, before building a stochastic model based on a combination of a spectral generator and of a non-Gaussian mapping. The particular case of polysilicon is then considered in the following three sections. First the micro-scale is studied in Section 4 . Based on experimental measurements reported in [23], the micro-scale structure, such as grain size and orientation distribution, is studied in terms of the Low Pressure Chemical Vapor Deposition (LPCVD) process temperature. The micro-scale thermo-mechanical properties are then defined in order to build the SVE realizations. Then, the meso-scale probabilistic material properties obtained from the stochastic homogenization process are compared to the ones generated by the defined stochastic model in Section 5. Finally, using the meso-scale random field realizations of the meso-scale thermo-mechanical properties, the structural behavior of MEMS resonator is studied in a probabilistic way in Section 6. Because of the efficiency of the method, it is possible to study several resonator geometries, and the effect of the boundary conditions and of the deposition temperatures, on the resonance quality factors of the resonators.

\section{Thermo-elasticity}

In this section, the governing equations of thermo-elasticity coupling, the corresponding weak formulation, and its finite element discretization, are first recalled. Thermo-mechanical stochastic finite elements are then described with a particular emphasis on the thermo-elastic damping effect in vibration analyses.

\subsection{Finite element formula for thermo-elasticity}

\subsubsection{Governing equations for thermo-elastic solids}

The equations that govern the motion of thermo-elastic solids include the balance laws for mass, momentum, and energy [43]. The weak form for linear coupled thermo-elastic problems can be derived from two governing equations. The first governing equation is the linear momentum balance equation. The second governing equation is derived from the balance of energy and the Clausius-Duhem inequality. Here we just give some resulted equations, more details are given in [20,43] and in Appendix A. 
The first governing equation is the linear momentum balance equation, which reads

$$
\rho \ddot{\boldsymbol{u}}-\nabla \cdot \boldsymbol{\sigma}-\rho \boldsymbol{b}=0,
$$

where $\rho$ is the mass density, $\boldsymbol{u}$ is the displacement vector, $\boldsymbol{\sigma}$ is the Cauchy stress tensor, and $\boldsymbol{b}$ is the body force density vector. The dot on top of a variable refers to its time derivative.

The second governing equation is obtained based on the balance of energy and the Clausius-Duhem inequality, which is stating the irreversibility of a natural process when energy dissipation is involved. When the only dissipation mechanism involved is heat conductivity, and no heat source is considered in the body, the balance of energy and the Clausius-Duhem relation lead to

$$
\dot{\mathcal{S}}=-\frac{\nabla \cdot \boldsymbol{q}}{T}
$$

where $\mathcal{S}$ is the entropy per unit volume of the body, $T$ is the absolute temperature, and $\boldsymbol{q}$ is the thermal flux vector, as detailed in Appendix A. In a general form, the Helmholtz free energy $\mathcal{F}$ of the body per unit volume is expressed in terms of the elastic potential $\psi(\varepsilon)$ per unit volume and satisfies

$$
\boldsymbol{\sigma}(\varepsilon, T)=\left(\frac{\partial \mathcal{F}}{\partial \varepsilon}\right)_{T} \text { and } \mathcal{S}(\varepsilon, T)=-\left(\frac{\partial \mathcal{F}}{\partial T}\right)_{\varepsilon}
$$

where $\varepsilon$ is the Cauchy strain tensor.

In the case of the absence of external force and of heat source, and at a temperature $T$ different from the reference temperature $T_{0}$, the Helmholtz free energy $\mathcal{F}$ accounting for the thermal expansion reads

$$
\mathcal{F}(\varepsilon, T)=\mathcal{F}_{0}(T)-\varepsilon: \frac{\partial^{2} \psi}{\partial \varepsilon \partial \varepsilon}: \boldsymbol{\alpha}\left(T-T_{0}\right)+\psi(\varepsilon),
$$

where $\boldsymbol{\alpha}=\frac{\partial \boldsymbol{\varepsilon}}{\partial T}$ is the thermal expansion tensor assumed to be constant with the temperature. From Eqs. (3), we have

$$
\boldsymbol{\sigma}(\varepsilon, T)=\left(\frac{\partial \mathcal{F}}{\partial \varepsilon}\right)_{T}=\frac{\partial^{2} \psi}{\partial \varepsilon \partial \varepsilon}: \varepsilon-\frac{\partial^{2} \psi}{\partial \varepsilon \partial \varepsilon}: \boldsymbol{\alpha}\left(T-T_{0}\right)
$$

and

$$
\mathcal{S}(\varepsilon, T)=-\left(\frac{\partial \mathcal{F}}{\partial T}\right)_{\varepsilon}=\mathcal{S}_{0}(T)+\varepsilon: \frac{\partial^{2} \psi}{\partial \varepsilon \partial \varepsilon}: \boldsymbol{\alpha},
$$

where $\mathcal{S}_{0}(T)=-\partial \mathcal{F}_{0} / \partial T$ is the entropy at $\varepsilon=\mathbf{0}$, i.e. at zero-deformation. Moreover, we can write $\partial \mathcal{S}_{0} / \partial t=\left(\partial \mathcal{S}_{0} / \partial T\right) \cdot(\partial T / \partial t)$, where the derivative $\left(\partial \mathcal{S}_{0} / \partial T\right)=\rho C_{v} / T$ with $C_{v}$ the heat capacity per unit mass at constant volume. Taking the derivative with respect to time $t$ of Eq. (6) and using Eq. (2) lead to the second governing equation, which reads

$$
\rho C_{v} \frac{\partial T}{\partial t}+T \boldsymbol{\alpha}: \frac{\partial^{2} \psi}{\partial \varepsilon \partial \varepsilon}: \frac{\partial \varepsilon}{\partial t}=\kappa: \frac{\partial^{2} T}{\partial \boldsymbol{x} \partial \boldsymbol{x}},
$$

where $\boldsymbol{\kappa}$ is the second order tensor called thermal conductivity assumed to be constant with the temperature, which satisfies $\boldsymbol{q}=-\boldsymbol{\kappa} \cdot \nabla T$. 


\subsubsection{Finite element discretization}

We assume that the temperature change corresponding to the mechanical loading is relatively small compared to the reference temperature $T_{0}$. Then we define $\vartheta=T-T_{0}$ and rewrite the two governing equations as

$$
\begin{aligned}
\rho \ddot{\boldsymbol{u}}-\nabla \cdot \boldsymbol{\sigma}-\rho \boldsymbol{b} & =0, \\
\rho C_{v} \dot{\vartheta}+T_{0} \boldsymbol{\alpha}: \mathbb{C}: \dot{\boldsymbol{\varepsilon}}-\boldsymbol{\kappa}: \frac{\partial^{2} \vartheta}{\partial \boldsymbol{x} \partial \boldsymbol{x}} & =0,
\end{aligned}
$$

where $\mathbb{C}=\frac{\partial^{2} \psi}{\partial \varepsilon \partial \varepsilon}$ and we use the approximation $T \approx T_{0}$ in the second term $T \boldsymbol{\alpha}: \mathbb{C}: \dot{\boldsymbol{\varepsilon}}$ of the second governing equation for the purpose of linearization. Indeed, as we intend to perform modal analyzes, linear equations are required. This set of equations is completed by the mechanical boundary conditions enforced on $\Gamma=\Gamma_{u} \cup \Gamma_{\mathcal{T}}$

$$
\begin{array}{ccc}
\boldsymbol{u}=\overline{\boldsymbol{u}} & \text { on } & \Gamma_{u}, \\
\boldsymbol{\sigma} \cdot \boldsymbol{n}=\boldsymbol{\mathcal { T }} & \text { on } & \Gamma_{\mathcal{T}},
\end{array}
$$

and by the thermal boundary conditions enforced on $\Gamma^{T}=\Gamma_{T}^{T} \cup \Gamma_{q}^{T}$

$$
\begin{array}{rlll}
T=\bar{T} & \text { on } & \Gamma_{T}^{T}, \\
\nabla T \cdot \boldsymbol{\kappa} \cdot \boldsymbol{n}=-\boldsymbol{q} \cdot \boldsymbol{n}=-\mathcal{Q} & \text { on } & \Gamma_{q}^{T},
\end{array}
$$

where $\overline{\boldsymbol{u}}$ is the constrained displacement field, $\mathcal{T}$ is the constrained surface traction, $\bar{T}$ is the constrained temperature, $\mathcal{Q}$ is the constrained thermal surface flux, and where $\boldsymbol{n}$ is the outward unit normal vector.

The weak form of the set of governing equations is established using suitable weight functions defined in the $n+1$ dimensional spaces:

$$
\begin{array}{cc}
\boldsymbol{w}_{u} \in\left[C^{0}\right]^{n} & \text { The weight function of the displacement field, } \\
w_{\vartheta} \in\left[C^{0}\right] & \text { The weight function of the temperature field } .
\end{array}
$$

Multiplying the governing equation (8) by the displacement weight function $\boldsymbol{w}_{u}$ and integrating the result over the domain $\Omega$ yields

$$
\int_{\Omega} \boldsymbol{w}_{u} \cdot[\rho \ddot{\boldsymbol{u}}-\nabla \cdot \boldsymbol{\sigma}-\rho \boldsymbol{b}] \mathrm{d} \Omega=0 .
$$

Applying the divergence theorem, the natural boundary conditions on $\Gamma_{\mathcal{T}}$, the essential boundary conditions on $\Gamma_{u}$, and the symmetry property of the Cauchy stress tensor leads to

$$
\int_{\Omega}\left\{\boldsymbol{w}_{u} \cdot \rho \ddot{\boldsymbol{u}}+\left[\nabla \boldsymbol{w}_{u}\right]^{\mathrm{T}}: \boldsymbol{\sigma}\right\} \mathrm{d} \Omega=\int_{\Omega} \rho \boldsymbol{w}_{u} \cdot \boldsymbol{b} \mathrm{d} \Omega+\int_{\Gamma \mathcal{T}} \boldsymbol{w}_{u} \cdot \mathcal{T} \mathrm{d} \Gamma .
$$

The same method is used to treat the second PDE (9), which results in

$$
\int_{\Omega}\left\{\rho C_{\vartheta} \dot{\vartheta} w_{\vartheta}+T_{0} \boldsymbol{\alpha}: \mathbb{C}: \dot{\boldsymbol{\varepsilon}} w_{\vartheta}+\nabla w_{\vartheta} \cdot \boldsymbol{\kappa} \cdot \nabla \vartheta\right\} \mathrm{d} \Omega=-\int_{\Gamma_{q}^{T}} \mathcal{Q} w_{\vartheta} \mathrm{d} \Gamma^{T}
$$


The third term on the left-hand side of Eq. (15), i.e. $\int_{\Omega} \nabla w_{\vartheta} \cdot \boldsymbol{\kappa} \cdot \nabla \vartheta \mathrm{d} \Omega$, is responsible for the thermo-elastic damping when the thermal relaxation time $t_{\chi}=\frac{\rho C_{v} v_{\chi}^{2}}{\|\boldsymbol{\kappa}\|_{\infty} \pi^{2}}$ is close to the deformation period. In this relation $l_{\chi}$ is the length characterizing the conduction process, typically the device thickness. When the thermal relaxation time $t_{\chi}$ is much smaller (larger) than the deformation period, the process is quasi-isothermal (quasi-adiabatic) and the the thermo-elastic damping is negligible.

The finite element discretization is straightforwardly formulated using the Galerkin approach. Toward this end, the displacement field $\boldsymbol{u}$ and the relative temperature field $\vartheta$ can be interpolated in each element $\Omega_{\mathrm{e}}$ using traditional shape function matrices $\boldsymbol{N}_{u}$ and $\boldsymbol{N}_{\vartheta}$ as follows:

$$
\boldsymbol{u}=\boldsymbol{N}_{u} \mathbf{u}, \quad \text { and } \quad \vartheta=\boldsymbol{N}_{\vartheta} \boldsymbol{\vartheta},
$$

where the vectors $\mathbf{u}$ and $\boldsymbol{\vartheta}$ contain the assembled nodal values of the displacements and of the relative temperature field, respectively. Similarly, the weight functions are interpolated using the same shape functions

$$
\boldsymbol{w}_{u}=\boldsymbol{N}_{u} \mathbf{d u}, \quad \text { and } \quad w_{\vartheta}=\boldsymbol{N}_{\vartheta} \mathbf{d} \boldsymbol{\vartheta},
$$

where $\mathbf{d u}$ and $\mathbf{d} \vartheta$ are arbitrary values fulfilling the essential boundary conditions.

The strain tensorial field and the gradient field of the relative temperature can easily be deduced, in terms of the problem unknowns, from

$$
\boldsymbol{\varepsilon}=\boldsymbol{B}_{u} \mathbf{u}, \quad \text { and } \quad \nabla \vartheta=\nabla \boldsymbol{N}_{\vartheta} \vartheta=\boldsymbol{B}_{\vartheta} \boldsymbol{\vartheta},
$$

where $\boldsymbol{B}_{u}$ and $\boldsymbol{B}_{\vartheta}$ represent the matrix strain operator and the matrix operator for the temperature gradient, respectively. The related derivatives with respect to time are

$$
\ddot{\boldsymbol{u}}=\boldsymbol{N}_{u} \ddot{\mathbf{u}}, \quad \dot{\varepsilon}=\boldsymbol{B}_{u} \dot{\mathbf{u}} \quad \text { and } \quad \dot{\vartheta}=\boldsymbol{N}_{\vartheta} \dot{\boldsymbol{\vartheta}}
$$

We recall the expression of stress, Eq. (5), in linear thermo-elasticity

$$
\boldsymbol{\sigma}=\mathbb{C}: \varepsilon-\mathbb{C}: \boldsymbol{\alpha} \vartheta .
$$

Therefore, using the arbitrary nature of $\mathbf{d} \mathbf{u}$ and $\mathbf{d} \boldsymbol{\vartheta}$, the equations (14) and (15) become

$$
\begin{array}{r}
\int_{\Omega}\left\{\rho \boldsymbol{N}_{u}^{\mathrm{T}} \boldsymbol{N}_{u} \ddot{\mathbf{u}}+\boldsymbol{B}_{u}^{\mathrm{T}} \boldsymbol{C} \boldsymbol{B}_{u} \mathbf{u}-\boldsymbol{B}_{u}^{\mathrm{T}} \boldsymbol{C} \boldsymbol{\alpha} \boldsymbol{N}_{\vartheta} \vartheta\right\} \mathrm{d} \Omega=\int_{\Omega} \boldsymbol{N}_{u}^{\mathrm{T}} \rho \boldsymbol{b} \mathrm{d} \Omega+\int_{\Gamma_{\mathcal{T}}} \boldsymbol{N}_{u}^{\mathrm{T}} \mathcal{T} \mathrm{d} \Gamma, \\
\int_{\Omega}\left\{\rho C_{\vartheta} \boldsymbol{N}_{\vartheta}^{\mathrm{T}} \boldsymbol{N}_{\vartheta} \dot{\boldsymbol{\vartheta}}+T_{0} \boldsymbol{N}_{\vartheta}^{\mathrm{T}} \boldsymbol{\alpha} \boldsymbol{C} \boldsymbol{B}_{u} \dot{\mathbf{u}}+\boldsymbol{B}_{\vartheta}^{\mathrm{T}} \boldsymbol{\kappa} \boldsymbol{B}_{\vartheta} \boldsymbol{\vartheta}\right\} \mathrm{d} \Omega=-\int_{\Gamma_{q}^{T}} \boldsymbol{N}_{\vartheta}^{\mathrm{T}} \mathcal{Q} \mathrm{d} \Gamma^{T},
\end{array}
$$

where $\boldsymbol{C}$ is the matrix form of the fourth order tensor $\mathbb{C}$.

The equations above can be stated in the following matrix form:

$$
\left[\begin{array}{cc}
\boldsymbol{M} & \mathbf{0} \\
\mathbf{0} & \mathbf{0}
\end{array}\right]\left[\begin{array}{c}
\ddot{\mathbf{u}} \\
\ddot{\boldsymbol{\vartheta}}
\end{array}\right]+\left[\begin{array}{cc}
\mathbf{0} & \mathbf{0} \\
\boldsymbol{D}_{\vartheta u} & \boldsymbol{D}_{\vartheta \vartheta}
\end{array}\right]\left[\begin{array}{c}
\dot{\mathbf{u}} \\
\dot{\boldsymbol{\vartheta}}
\end{array}\right]+\left[\begin{array}{cc}
\boldsymbol{K}_{u u} & \boldsymbol{K}_{u \vartheta} \\
\mathbf{0} & \boldsymbol{K}_{\vartheta \vartheta}
\end{array}\right]\left[\begin{array}{c}
\mathbf{u} \\
\boldsymbol{\vartheta}
\end{array}\right]=\left[\begin{array}{l}
\boldsymbol{F}_{u} \\
\boldsymbol{F}_{\vartheta}
\end{array}\right],
$$

where the definitions of all the sub-matrices can be found in Appendix B. 


\subsection{Stochastic formulation of thermo-elastic problems}

We consider the uncertainties resulting from the heterogeneous micro-structure, which in turn result in uncertainties in the material properties. We recall the main equations in finite element analysis, equations (21), (22) and their matrix form (23). The material property items are the elastic tensor $\boldsymbol{C}$, heat conductivity tensor $\boldsymbol{\kappa}$, and thermal expansion tensor $\boldsymbol{\alpha}$ which can be represented under the form of a random field. Using the Voigt notation for the different tensors, we have the random fields of the elasticity tensor, heat conductivity tensor, and thermal expansion tensor $\boldsymbol{C}(\boldsymbol{x}, \boldsymbol{\theta}): \Omega \times \mathcal{P} \rightarrow \boldsymbol{M}_{6}^{+}(R), \boldsymbol{\kappa}(\boldsymbol{x}, \boldsymbol{\theta}): \Omega \times \mathcal{P} \rightarrow \boldsymbol{M}_{3}^{+}(R)$, and $\boldsymbol{\alpha}(\boldsymbol{x}, \boldsymbol{\theta}): \Omega \times \mathcal{P} \rightarrow \boldsymbol{M}_{3}(R)$ respectively, over the spatial domain $\Omega$, which are functions of the spatial coordinate $\boldsymbol{x} . \boldsymbol{\theta} \in \mathcal{P}$ denotes the elements in the sample space involving random quantities, $\boldsymbol{M}_{N}^{+}(R)$ refers to the set of all symmetric positive-definite real matrices of size $N \times N$, and $\boldsymbol{M}_{N}(R)$ refers to the set of all symmetric real matrices of size $N \times N$ in order to keep $\boldsymbol{\alpha}(\boldsymbol{x}, \boldsymbol{\theta})$ more general (negative thermal expansion is possible for some materials).

Because all these three tensors relate to the heterogeneities or micro structures of materials, it is not proper to write them as three uncorrelated random fields. Using the symmetric properties of $\boldsymbol{C}, \boldsymbol{\kappa}$, and $\boldsymbol{\alpha}$, we can assemble all the entries of their low triangular matrices into a vector $\boldsymbol{V}$ of dimension 33, in which there are 21 entries for the elastic tensor $\boldsymbol{C}, 6$ for the conductivity tensor $\boldsymbol{\kappa}$, and 6 for the thermal expansion tensor $\boldsymbol{\alpha}$. Then, we define a random field $\boldsymbol{V}(\boldsymbol{x}, \boldsymbol{\theta}): \Omega \times \mathcal{P} \rightarrow \boldsymbol{S}$ with $^{1}$

$$
\boldsymbol{S}=\left\{\mathbf{v} \in \mathbb{R}^{33}: \boldsymbol{C}(\mathbf{v}) \in \boldsymbol{M}_{6}^{+}(R), \boldsymbol{\kappa}(\mathbf{v}) \in \boldsymbol{M}_{3}^{+}(R), \boldsymbol{\alpha}(\mathbf{v}) \in \boldsymbol{M}_{3}(R)\right\}
$$

As a result, the finite element formulation (23) is restated in the probabilistic form as

$$
\left[\begin{array}{cc}
\boldsymbol{M} & \mathbf{0} \\
\mathbf{0} & \mathbf{0}
\end{array}\right]\left[\begin{array}{c}
\ddot{\mathbf{u}} \\
\ddot{\boldsymbol{\vartheta}}
\end{array}\right]+\left[\begin{array}{cc}
\mathbf{0} & \mathbf{0} \\
\boldsymbol{D}_{\vartheta u}(\mathbf{v}) & \boldsymbol{D}_{\vartheta \vartheta}(\mathbf{v})
\end{array}\right]\left[\begin{array}{c}
\dot{\mathbf{u}} \\
\dot{\boldsymbol{\vartheta}}
\end{array}\right]+\left[\begin{array}{cc}
\boldsymbol{K}_{u u}(\mathbf{v}) & \boldsymbol{K}_{u \vartheta}(\mathbf{v}) \\
\mathbf{0} & \boldsymbol{K}_{\vartheta \vartheta}(\mathbf{v})
\end{array}\right]\left[\begin{array}{l}
\mathbf{u} \\
\boldsymbol{\vartheta}
\end{array}\right]=\left[\begin{array}{l}
\boldsymbol{F}_{u} \\
\boldsymbol{F}_{\vartheta}
\end{array}\right],
$$

where the definitions of all the sub-matrices can be found in Appendix B.

An accurate SFEM analysis with the simple point discretization of the random field requires that the mesh elements must be small enough compared to the correlation length [6]. In 1D, the correlation length of a stationary random field is defined by [37]

$$
l_{\mathbf{C}}=\frac{\int_{-\infty}^{\infty} R(\tau) \mathrm{d} \tau}{R(0)}
$$

where $R(\tau)$ is the correlation function of the considered random value.

In Section 3, we will introduce an intermediate scale, the meso-scale, and detail how to extract the meso-scale random field of vector $\boldsymbol{V}$ from the finite element resolution of meso-scale volume elements. The

\footnotetext{
${ }^{1}$ We use the notation $\boldsymbol{V}$ to refer to the random field, and the notation $\mathbf{v}$ to refer to a sample or realization of the random field $\boldsymbol{V}$
} 
obtained smooth random field description for the material properties has thus a correlation length larger than the characteristic length of micro structure, and allows the use of coarser elements in the SFEM at the structural-scale.

\subsection{Numerical evaluation of the Quality-factor}

In a wide variety of MEMS devices, such as in accelerometers, gyrometers, sensors, charge detectors, radio-frequency filters, magnetic resonance force microscopes, or again torque magnetometers, the resonator part is a critical component for which a high quality factor $Q$ is sought[20]. The $\mathrm{Q}$ factor is defined by the ratio of the stored energy in the resonator $W$ and the total dissipated energy $\Delta W$ per cycle of vibration

$$
Q=2 \pi \frac{W}{\Delta W} .
$$

The thermo-elastic damping represents the energy loss associated to an entropy rise caused by the coupling between the heat transfer and strain rate. In solids with a positive thermal expansion effect, an increase of temperature induces a dilatation and inversely, a decrease of temperature produces a compression. Similarly, a dilatation lowers the temperature and a compression raises it. Therefore, when a thermo-elastic solid is in motion and taken out of equilibrium, the energy dissipates through the irreversible flow of heat driven by local temperature gradients that are generated by the strain field through its coupling with the temperature field.

Thermo-elastic coupling induces damping whose effect is characterized by a resonance frequency shift [21]. The quality factor can be computed through solving the eigenvalues of the coupled problem using the finite element method. The dissipation of the resonating beam is measured by the fraction of energy loss per cycle, which is the inverse of the quality factor, $Q^{-1}$, and can be expressed in terms of the imaginary and real parts of the pulsation as

$$
Q_{i}^{-1}=\frac{2\left|\operatorname{Im}\left(\varpi_{i}\right)\right|}{\sqrt{\operatorname{Re}^{2}\left(\varpi_{i}\right)+\operatorname{Im}^{2}\left(\varpi_{i}\right)}},
$$

where $\varpi_{i}$ is the thermo-elastic resonant pulsation of the $i^{\text {th }}$ mode. As the imaginary part of the resonant pulsation considered is much smaller than the real part, and as the first mode carries most of the energy, the approximated inverse of the quality factor is associated to the first mode and reads

$$
Q^{-1} \approx 2\left|\frac{\operatorname{Im}\left(\varpi_{1}\right)}{\operatorname{Re}\left(\varpi_{1}\right)}\right| .
$$

Equation (25) can be rewritten in general as

$$
\mathbb{M} \ddot{\boldsymbol{v}}+\mathbb{D}(\mathbf{v}) \dot{\boldsymbol{v}}+\mathbb{K}(\mathbf{v}) \boldsymbol{v}=\mathbb{F},
$$

where $\boldsymbol{v}=\{\mathbf{u}, \boldsymbol{\vartheta}\}^{\mathrm{T}}$. To calculate the effect of thermo-elastic coupling on the vibrations of a structure, we solve the coupled thermo-elastic equations (30) for the case of harmonic vibrations, and we set

$$
\boldsymbol{v}=\boldsymbol{v}_{0} e^{i \varpi t},
$$


in order to obtain the normal modes of vibration and their corresponding frequencies. In general the frequencies are complex, the real part $\operatorname{Re}(\varpi)$ giving the new eigenvalue frequencies of the structure in the presence of thermo-elastic coupling, and the imaginary part $\operatorname{Im}(\varpi)$ giving the attenuation of the vibration. The quality factor, Eq. (29), can be computed from the eigenvalue of the coupled problem as

$$
Q=\frac{1}{2}\left|\frac{\operatorname{Re}\left(\varpi_{1}\right)}{\operatorname{Im}\left(\varpi_{1}\right)}\right| .
$$

The general quadratic eigenvalue problem to solve results from Eq. (30) without considering external force and external heat exchange. For simplicity, we rewrite $\lambda=i \varpi$. Submitting Eq. (31) into Eq. (30) and setting its right hand side to be zero results in

$$
\left(\mathbb{M} \lambda^{2}+\mathbb{D}(\mathbf{v}) \lambda+\mathbb{K}(\mathbf{v})\right) \boldsymbol{v}=\mathbf{0} .
$$

A generation transformation is applied to convert the original quadratic problem into a first-order problem as

$$
\left[\begin{array}{cc}
-\mathbb{K}(\mathbf{v}) & \mathbf{0} \\
\mathbf{0} & \mathbb{I}
\end{array}\right]\left[\begin{array}{l}
\boldsymbol{v} \\
\dot{v}
\end{array}\right]=\lambda\left[\begin{array}{cc}
\mathbb{D}(\mathbf{v}) & \mathbb{M} \\
\mathbb{I} & \mathbf{0}
\end{array}\right]\left[\begin{array}{c}
\boldsymbol{v} \\
\dot{v}
\end{array}\right] .
$$

Expending all the entries of this compact allows this last equation to be written under the form

$$
\left[\begin{array}{cccc}
-K_{u u}(\mathbf{v}) & -K_{u \vartheta}(\mathbf{v}) & \mathbf{0} & 0 \\
\mathbf{0} & -\boldsymbol{K}_{\vartheta \vartheta}(\mathbf{v}) & \mathbf{0} & \mathbf{0} \\
\mathbf{0} & \mathbf{0} & \boldsymbol{I} & \mathbf{0} \\
\mathbf{0} & \mathbf{0} & \mathbf{0} & \boldsymbol{I}
\end{array}\right]\left[\begin{array}{c}
\mathbf{u} \\
\vartheta \\
\dot{\boldsymbol{u}} \\
\dot{\boldsymbol{\vartheta}}
\end{array}\right]=\lambda\left[\begin{array}{cccc}
\mathbf{0} & \mathbf{0} & \boldsymbol{M} & \mathbf{0} \\
\boldsymbol{D}_{\vartheta u}(\mathbf{v}) & D_{\vartheta \vartheta}(\mathbf{v}) & \mathbf{0} & \mathbf{0} \\
\boldsymbol{I} & \mathbf{0} & \mathbf{0} & \mathbf{0} \\
\mathbf{0} & \boldsymbol{I} & \mathbf{0} & \mathbf{0}
\end{array}\right]\left[\begin{array}{c}
\mathbf{u} \\
\boldsymbol{\vartheta} \\
\dot{\mathbf{u}} \\
\dot{\boldsymbol{\vartheta}}
\end{array}\right]
$$

where the eigenvalues associated with the fourth matrix equation are independent of the three other ones and can be eliminated without affecting the eigenvalue problem. The problem that needs to be solved is thus

$$
\left[\begin{array}{ccc}
-\boldsymbol{K}_{u u}(\mathbf{v}) & -\boldsymbol{K}_{u \vartheta}(\mathbf{v}) & \mathbf{0} \\
\mathbf{0} & -\boldsymbol{K}_{\vartheta \vartheta}(\mathbf{v}) & \mathbf{0} \\
\mathbf{0} & \mathbf{0} & \boldsymbol{I}
\end{array}\right]\left[\begin{array}{c}
\mathbf{u} \\
\vartheta \\
\dot{\mathbf{u}}
\end{array}\right]=\lambda\left[\begin{array}{ccc}
\mathbf{0} & \mathbf{0} & \boldsymbol{M} \\
\boldsymbol{D}_{\vartheta u}(\mathbf{v}) & \boldsymbol{D}_{\vartheta \vartheta}(\mathbf{v}) & \mathbf{0} \\
\boldsymbol{I} & \mathbf{0} & \mathbf{0}
\end{array}\right]\left[\begin{array}{c}
\mathbf{u} \\
\vartheta \\
\dot{\mathbf{u}}
\end{array}\right],
$$

which can be written under the simpler form

$$
\boldsymbol{A}(\mathbf{v}) \boldsymbol{p}=\lambda \boldsymbol{B}(\mathbf{v}) \boldsymbol{p},
$$

where $\boldsymbol{p}=\{\mathbf{u}, \boldsymbol{\vartheta}, \dot{\mathbf{u}}\}^{\mathrm{T}}$. The details on how to solve this eigenvalue problem were discussed in [20] and [18].

After solving this eigenvalue problem, the quality factor realizations can thus be computed from equation (32) using the relation of $\varpi$ and $\lambda$, which gives

$$
Q(\mathbf{v})=\frac{1}{2}\left|\frac{\operatorname{Im}\left(\lambda_{1}\right)}{\operatorname{Re}\left(\lambda_{1}\right)}\right| .
$$




\section{Stochastic homogenization: from the micro-scale to the meso-scale}

In order to formulate the problem (25), the meso-scale random vector field $\boldsymbol{V}$ needs to be defined. In this section we explain how to generate the meso-scale random vector field $\boldsymbol{V}$ by, first performing computational homogenization on meso-scale volume element -or SVE- realizations, and then developing a stochastic generator using the homogenized properties realizations as input.

\subsection{Evaluation of the apparent meso-scale properties tensors}

The apparent -or homogenized- meso-scale material tensors can be estimated from the finite element resolution of a meso-scale boundary value problem. The homogenized elastic material operator was extracted from the stiffness matrix of the FE model in [17,30]. More recently, the problem of thermo-elasticity was formulated and discussed in $[33,40]$. In the following we rewrite the thermo-elasticity scale transition equations, with a particular emphasis on the extraction of the material operators of the homogenized thermoelastic properties using the multiple-constraint projection method described in [1].

\subsubsection{Definition of scales transition}

The homogenization of thermo-elastic problems is summarized hereafter. First of all, in the homogenization process of the thermo-mechanical problem, the two requirements which state the separation of the macro- and meso-scales and the thermal steady-state in the SVE read:

1. The SVE $\omega$ should be small enough for the time of the strain wave to propagate in the SVE to remain negligible, so that the equivalence of the micro-strain to the macro-strain is instantaneous. This assumption allows writing

$$
\nabla_{\mathrm{m}} \cdot \boldsymbol{\sigma}_{\mathrm{m}}=\mathbf{0} \quad \text { in } \omega
$$

where the subscript ' $m$ ' refers to the local value at the micro-scale.

2. The SVE should be small enough for the time variation of heat storage to remain negligible. This assumption corresponds to the thermal steady-state of micro-scale, which is expressed as

$$
\nabla_{\mathrm{m}} \cdot \boldsymbol{q}_{\mathrm{m}}=0 \quad \text { in } \omega
$$

These two scale-separation requirements hold in the vibration problem of micro resonator as the SVEs are by definition of reduced sizes. Therefore, the finite element formulation of the meso-scale BVP is similar to Eq. (23), but stated in a steady state, and reads

$$
\left[\begin{array}{cc}
\boldsymbol{K}_{u u} & \boldsymbol{K}_{u \vartheta} \\
\mathbf{0} & \boldsymbol{K}_{\vartheta \vartheta}
\end{array}\right]\left[\begin{array}{l}
\mathbf{u} \\
\boldsymbol{\vartheta}
\end{array}\right]=\left[\begin{array}{l}
\boldsymbol{F}_{u} \\
\boldsymbol{F}_{\vartheta}
\end{array}\right] .
$$


Within a multi-scale framework, macro-scale values can be defined as the volume average of a micro-scale field on the meso-scale volume-element $\omega$, following

$$
a_{\mathrm{M}}=<a_{\mathrm{m}}>=\frac{1}{V(\omega)} \int_{\omega} a_{\mathrm{m}} \mathrm{d} V
$$

where the subscript 'M' refers to the homogenized value on the SVE, $\langle\bullet\rangle$ is the volume average, and $V(\omega)$ is the volume of the meso-scale volume element $\omega$. The following homogenized values on the SVE need to be consistent with their micro values:

- The mass density:

$$
\rho_{\mathrm{M}}=<\rho_{\mathrm{m}}>
$$

- The heat capacity at constant volume $C_{v}$, which has to satisfy the consistency of heat capacity at the different scales:

$$
\rho_{\mathrm{M}} C_{v \mathrm{M}}=<\rho_{\mathrm{m}} C_{v \mathrm{~m}}>\quad \text { and } \quad C_{v \mathrm{M}}=\frac{<\rho_{\mathrm{m}} C_{v \mathrm{~m}}>}{<\rho_{\mathrm{m}}>}
$$

- The stress and strain tensors:

$$
\begin{aligned}
\boldsymbol{\sigma}_{\mathrm{M}} & =<\boldsymbol{\sigma}_{\mathrm{m}}>=<\mathbb{C}_{\mathrm{m}}: \boldsymbol{\varepsilon}_{\mathrm{m}}-\mathbb{C}_{\mathrm{m}}: \boldsymbol{\alpha}_{\mathrm{m}} \vartheta_{\mathrm{m}}> \\
& =\mathbb{C}_{\mathrm{M}}: \varepsilon_{\mathrm{M}}-\mathbb{C}_{\mathrm{M}}: \boldsymbol{\alpha}_{\mathrm{M}} \vartheta_{\mathrm{M}}
\end{aligned}
$$

and

$$
\varepsilon_{\mathrm{M}}=\left(\frac{\nabla_{\mathrm{M}} \otimes \boldsymbol{u}_{\mathrm{M}}+\left(\nabla_{\mathrm{M}} \otimes \boldsymbol{u}_{\mathrm{M}}\right)^{\mathrm{T}}}{2}\right)=<\varepsilon_{\mathrm{m}}>
$$

- The heat flux and temperature gradient:

$$
\boldsymbol{q}_{\mathrm{M}}=<\boldsymbol{q}_{\mathrm{m}}>
$$

and

$$
\nabla_{\mathrm{M}} \vartheta_{\mathrm{M}}=<\nabla_{\mathrm{m}} \vartheta_{\mathrm{m}}>
$$

In order to respect the energy consistency at the different scales, the following conditions have also to be respected:

- The consistency of deformation energy at the different scales:

$$
\begin{aligned}
\boldsymbol{\sigma}_{\mathrm{M}}: \delta \varepsilon_{\mathrm{M}} & =\delta \varepsilon_{\mathrm{M}}: \mathbb{C}_{\mathrm{M}}: \boldsymbol{\varepsilon}_{\mathrm{M}}-\delta \varepsilon_{\mathrm{M}}: \mathbb{C}_{\mathrm{M}}: \boldsymbol{\alpha}_{\mathrm{M}} \vartheta_{\mathrm{M}} \\
& =<\delta \varepsilon_{\mathrm{m}}: \mathbb{C}_{\mathrm{m}}: \varepsilon_{\mathrm{m}}-\delta \varepsilon_{\mathrm{m}}: \mathbb{C}_{\mathrm{m}}: \boldsymbol{\alpha}_{\mathrm{m}} \vartheta_{\mathrm{m}}>
\end{aligned}
$$

for any temperature $\vartheta_{\mathrm{M}}$ or deformation field $\varepsilon_{\mathrm{M}}$. 
- The consistency of entropy change at the different scales is obtained for infinitesimal temperature changes ${ }^{2}$ :

$$
\boldsymbol{q}_{\mathrm{M}} \cdot \nabla_{\mathrm{M}} \delta \vartheta_{\mathrm{M}}=<\boldsymbol{q}_{\mathrm{m}} \cdot \nabla_{\mathrm{m}} \delta \vartheta_{\mathrm{m}}>
$$

- The consistency of heat storage at the micro- and macro-scales:

$$
\rho_{\mathrm{M}} C_{v \mathrm{M}} \vartheta_{\mathrm{M}}=<\rho_{\mathrm{m}} C_{v \mathrm{~m}} \vartheta_{\mathrm{m}}>
$$

Considering a first order homogenization process, the micro-scale fields are defined as

$$
\begin{aligned}
& \boldsymbol{u}_{\mathrm{m}}(\boldsymbol{x})=\left(\boldsymbol{u}_{\mathrm{M}} \otimes \nabla_{\mathrm{M}}\right) \cdot\left(\boldsymbol{x}-\boldsymbol{x}_{\mathrm{ref}}\right)+\boldsymbol{u}^{\prime}(\boldsymbol{x}), \\
& \vartheta_{\mathrm{m}}(\boldsymbol{x})=\vartheta_{\mathrm{ref}}+\nabla_{\mathrm{M}} \vartheta_{\mathrm{M}} \cdot\left(\boldsymbol{x}-\boldsymbol{x}_{\mathrm{ref}}\right)+\vartheta^{\prime}(\boldsymbol{x}),
\end{aligned}
$$

where $\boldsymbol{u}^{\prime}$ and $\vartheta^{\prime}$ are the perturbation fields. To satisfy Eqs. (46) and (48), the following respective conditions should be satisfied

$$
\begin{aligned}
& 0=<\nabla_{\mathrm{m}} \boldsymbol{u}^{\prime}(\boldsymbol{x})>=\frac{1}{V(\omega)} \int_{\partial \omega} \boldsymbol{u}^{\prime} \otimes \boldsymbol{n} \mathrm{d} S, \\
& 0=<\nabla_{\mathrm{m}} \vartheta^{\prime}(\boldsymbol{x})>=\frac{1}{V(\omega)} \int_{\partial \omega} \vartheta^{\prime} \boldsymbol{n} \mathrm{d} S,
\end{aligned}
$$

where $\boldsymbol{n}$ is the normal of the boundary $\partial \omega$.

Finally, in order to satisfy the energy and entropy change consistency conditions, using Eqs. (52) and (53) in respectively Eqs. (49) and (50), integrating by parts and using the equilibrium Eqs. (39) and (40), lead to

$$
\begin{aligned}
0 & =\int_{\partial \omega}\left(\boldsymbol{\sigma}_{\mathrm{m}} \cdot \boldsymbol{n}\right) \cdot \delta \boldsymbol{u}^{\prime} \mathrm{d} S \\
0 & =\int_{\partial \omega}\left(\boldsymbol{q}_{\mathrm{m}} \cdot \boldsymbol{n}\right) \delta \vartheta^{\prime} \mathrm{d} S .
\end{aligned}
$$

Under the conditions (54-55) and (56-57), the homogenized elastic tensor $\mathbb{C}_{\mathrm{M}}$, thermal conductivity tensor $\boldsymbol{\kappa}_{\mathrm{M}}$ and thermal expansion tensor $\boldsymbol{\alpha}_{\mathrm{M}}$ of SVE need to be sought out by solving the BVP on SVE.

\subsubsection{Definition of the constrained micro-scale finite element problem}

The specified kinematic admissible boundary conditions applied on a finite element discretization (41) of the SVE in this work are the Periodic Boundary Conditions (PBCs) of displacement $\boldsymbol{u}_{\mathrm{m}}$ and temperature $\vartheta_{\mathrm{m}}$, which read

$$
\begin{aligned}
& \boldsymbol{u}_{\mathrm{m}}\left(\boldsymbol{x}^{+}\right)-\boldsymbol{u}_{\mathrm{m}}\left(\boldsymbol{x}^{-}\right)=\left(\boldsymbol{u}_{\mathrm{M}} \otimes \nabla_{\mathrm{M}}\right) \cdot\left(\boldsymbol{x}^{+}-\boldsymbol{x}^{-}\right), \\
& \vartheta_{\mathrm{m}}\left(\boldsymbol{x}^{+}\right)-\vartheta_{\mathrm{m}}\left(\boldsymbol{x}^{-}\right)=\nabla_{\mathrm{M}} \vartheta_{\mathrm{M}} \cdot\left(\boldsymbol{x}^{+}-\boldsymbol{x}^{-}\right), \\
& \forall \boldsymbol{x}^{+} \in \partial \omega^{+} \text {and corresponding } \boldsymbol{x}^{-} \in \partial \omega^{-},
\end{aligned}
$$

\footnotetext{
${ }^{2}$ for finite temperature changes, this last relation is an approximation of $\frac{q_{\mathrm{M}} \cdot \nabla_{\mathrm{M}} \delta \vartheta_{\mathrm{M}}}{T_{\mathrm{M}}}=\left\langle\frac{\boldsymbol{q}_{\mathrm{m}} \cdot \nabla_{\mathrm{m}} \delta \vartheta_{\mathrm{m}}}{T_{\mathrm{m}}}\right\rangle$
} 
where the parallelepiped SVE faces have been separated in opposite surfaces $\partial \omega^{-}$and $\partial \omega^{+}$. These boundary conditions are completed by the consistency condition (51).

Other kinds of boundary conditions can be considered in order to satisfy (54-55) and (56-57), such as the Kinematically Uniform Boundary Conditions or KUBCs $\left(\boldsymbol{u}^{\prime}(\boldsymbol{x})=0\right.$ and $\delta \vartheta^{\prime}=0$ on $\left.\partial \omega\right)$, the Static Uniform Boundary Conditions or SUBCs $\left(\boldsymbol{\sigma}_{\mathrm{m}} \cdot \boldsymbol{n}=\boldsymbol{\sigma}_{\mathrm{M}} \cdot \boldsymbol{n}\right.$ and $\boldsymbol{q}_{\mathrm{m}} \cdot \boldsymbol{n}=\boldsymbol{q}_{\mathrm{M}} \cdot \boldsymbol{n}$ on $\left.\partial \omega\right)$ ), or a suitable combination of those under the form of Mixed Boundary Conditions or MBCs. Compared to PBCs, while KUBCs correspond to a more constrained system, leading to a stiffer response, SUBCs correspond to a less constrained system, leading to a more compliant response. Therefore PBCs are usually considered during the homogenization process, including for non-periodic micro-structures [16, 19, 41]. An alternative to PBCs for non-periodic micro-structures is the use of MBCs in which a part of the displacement field is kinematically constrained and the other part is statically constrained. However in the present case, as the thermal field involves only one degree of freedom, the MBCs cannot be applied. In order to apply the PBCs on non-periodic micro-structures as for polycrystalline materials, and thus on a non-periodic mesh, we have recourse to a polynomial interpolation of the unknowns fields on the boundary, as detailed in [31].

The kinematics constraints are defined by $\mathbb{F}_{\mathrm{M}}=\boldsymbol{u}_{\mathrm{M}} \otimes \nabla_{\mathrm{M}}$ the macroscopic displacement gradient, with $\boldsymbol{F}_{\mathrm{M}}$ the nine components of $\mathbb{F}_{\mathrm{M}}$ written under a vectorial form, by $\nabla_{\mathrm{M}} \vartheta_{\mathrm{M}}$ the macroscopic temperature gradient, and by $\vartheta_{\mathrm{M}}$ the macroscopic temperature. These kinematics constraints can be grouped under the vector $\boldsymbol{K}_{\mathrm{M}}^{\mathrm{T}}=\left[\begin{array}{lll}\boldsymbol{F}_{\mathrm{M}}^{\mathrm{T}} & \nabla_{\mathrm{M}} \vartheta_{M}^{\mathrm{T}} & \vartheta_{\mathrm{M}}\end{array}\right]$.

Dropping the subscript 'm' for simplicity, the degrees of freedom (dofs) are separated in constrained dofs, such as the nodal displacements $\boldsymbol{u}_{c}$ at the corner nodes, in dependent dofs, which relates to the periodic boundary conditions (58) and to the heat consistency (51), such as the nodal displacements $\boldsymbol{u}_{b}$ at the boundary, the nodal temperatures $\boldsymbol{\vartheta}_{c}$ at the corners, $\boldsymbol{\vartheta}_{b}$ at the boundary, and $\boldsymbol{\vartheta}_{i}$ inside the volume element, and in independent dofs as the nodal displacements $\boldsymbol{u}_{i}$ inside the volume element, see Appendix C. Therefore, on the one hand, the micro-structural problem (41) is organized in terms of the nodal unknowns

$$
\boldsymbol{\varphi}=\left[\begin{array}{lll}
\boldsymbol{u}_{c}^{\mathrm{T}} & \boldsymbol{\varphi}_{b}^{\mathrm{T}} & \boldsymbol{u}_{i}^{\mathrm{T}}
\end{array}\right]^{\mathrm{T}}
$$

with $\boldsymbol{\varphi}_{b}^{\mathrm{T}}=\left[\boldsymbol{u}_{b}^{\mathrm{T}} \boldsymbol{\vartheta}_{c}^{\mathrm{T}} \boldsymbol{\vartheta}_{b}^{\mathrm{T}} \boldsymbol{\vartheta}_{i}^{\mathrm{T}}\right]$. On the other hand, the boundary conditions (58) and the heat consistency (51) are expressed as

$$
\begin{aligned}
0 & =\boldsymbol{u}_{c}-\boldsymbol{S}_{\varphi_{c}} \boldsymbol{K}_{\mathrm{M}}, \\
0 & =\boldsymbol{C}_{\varphi_{c}} \boldsymbol{u}_{c}+\boldsymbol{C}_{\varphi_{b}} \boldsymbol{\varphi}_{b}-\boldsymbol{S}_{\varphi_{b}} \boldsymbol{K}_{\mathrm{M}},
\end{aligned}
$$

where the constraints matrices $\boldsymbol{C}$ and $\boldsymbol{S}$ are detailed in Appendix C. Note that these expressions remain valid for non-periodic meshes when using the interpolant method developed in [31]. 


\subsubsection{Resolution of the constrained micro-scale finite element problem}

The resolution of the constrained micro-scale BVP follows the multiple-constraint projection method described in [1] and the condensation method developed in [30]. The functional related to the constrained micro-scale problem (41) completed by the conditions (60-61) reads

$$
\Psi=\frac{1}{2} \boldsymbol{\varphi}^{\mathrm{T}} \boldsymbol{K} \boldsymbol{\varphi}-\left[\boldsymbol{u}_{c}-\boldsymbol{S}_{\varphi_{c}} \boldsymbol{K}_{\mathrm{M}}\right]^{\mathrm{T}} \boldsymbol{\lambda}_{u_{c}}-\left[\boldsymbol{C}_{\varphi_{c}} \boldsymbol{u}_{c}+\boldsymbol{C}_{\varphi_{b}} \boldsymbol{\varphi}_{b}-\boldsymbol{S}_{\varphi_{b}} \boldsymbol{K}_{\mathrm{M}}\right]^{\mathrm{T}} \boldsymbol{\lambda}_{\varphi_{b}},
$$

where the Lagrange multipliers $\boldsymbol{\lambda}_{u_{c}}$ and $\boldsymbol{\lambda}_{\varphi_{b}}$ are respectively related to the corner displacement constraints (60) and to the dependent unknowns constraints (61).

The solution of the problem corresponds to the stationary point of Eq. (62) with respect to the nodal unknowns, leading to the expression of the Lagrange multipliers $\boldsymbol{\lambda}_{\varphi_{b}}$ and $\boldsymbol{\lambda}_{u_{c}}$, see Appendix C.2.

The homogenized stress tensor (45) and the homogenized thermal flux vector (47) can then be evaluated in the vectorial form as

$$
\begin{aligned}
\boldsymbol{\Sigma}_{\mathrm{M}} & =\frac{1}{V(\omega)}\left(\frac{\partial \Psi}{\partial \boldsymbol{F}_{\mathrm{M}}}\right)=\frac{1}{V(\omega)}\left[\begin{array}{lll}
\boldsymbol{I}_{9 \times 9} & \mathbf{0}_{9 \times 3} & \mathbf{0}_{9 \times 1}
\end{array}\right]\left\{\boldsymbol{S}_{\varphi_{c}}^{\mathrm{T}} \boldsymbol{\lambda}_{u_{c}}+\boldsymbol{S}_{\varphi_{b}}^{\mathrm{T}} \boldsymbol{\lambda}_{\varphi_{b}}\right\} \\
\boldsymbol{q}_{\mathrm{M}} & =\frac{1}{V(\omega)}\left(\frac{\partial \Psi}{\partial \nabla_{\mathrm{M}} \vartheta_{\mathrm{M}}}\right)=\frac{1}{V(\omega)}\left[\begin{array}{lll}
\mathbf{0}_{3 \times 9} & \boldsymbol{I}_{3 \times 3} & \mathbf{0}_{3 \times 1}
\end{array}\right]\left\{\boldsymbol{S}_{\varphi_{c}}^{\mathrm{T}} \boldsymbol{\lambda}_{u_{c}}+\boldsymbol{S}_{\varphi_{b}}^{\mathrm{T}} \boldsymbol{\lambda}_{\varphi_{b}}\right\}
\end{aligned}
$$

respectively, see Appendix C.3 for details.

To compute the material operators, the stationary point of (62) is linearized with respect to the kinematics constraints $\boldsymbol{K}_{\mathrm{M}}$, yielding the variations of the Lagrange multipliers $\delta \boldsymbol{\lambda}_{\varphi_{b}}$ and $\delta \boldsymbol{\lambda}_{u_{c}}$ in terms of the variation $\delta \boldsymbol{K}_{\mathrm{M}}$, see Appendix C.3 for details. The variations of the homogenized stress (63) and of the homogenized heat flux (64) thus yield the apparent elasticity tensor $\boldsymbol{C}_{\mathrm{M}}=\frac{\partial \boldsymbol{\Sigma}_{\mathrm{M}}}{\partial \boldsymbol{F}_{\mathrm{M}}}$ in the matrix form following

$$
\boldsymbol{C}_{\mathrm{M}}=\frac{1}{V(\omega)}\left[\begin{array}{lll}
\boldsymbol{I}_{9 \times 9} & \mathbf{0}_{9 \times 3} & \mathbf{0}_{9 \times 1}
\end{array}\right]\left\{\boldsymbol{S}_{\varphi_{c}}^{\mathrm{T}} \frac{\partial \boldsymbol{\lambda}_{u_{c}}}{\partial \boldsymbol{K}_{\mathrm{M}}}+\boldsymbol{S}_{\varphi_{b}}^{\mathrm{T}} \frac{\partial \boldsymbol{\lambda}_{\varphi_{b}}}{\partial \boldsymbol{K}_{\mathrm{M}}}\right\}\left[\begin{array}{c}
\boldsymbol{I}_{9 \times 9} \\
\mathbf{0}_{3 \times 9} \\
\mathbf{0}_{1 \times 9}
\end{array}\right],
$$

the homogenized conductivity tensor $\boldsymbol{\kappa}_{\mathrm{M}}=-\frac{\partial \boldsymbol{q}_{\mathrm{M}}}{\partial \nabla_{M} \vartheta_{\mathrm{M}}}$ in the matrix form following

$$
\boldsymbol{\kappa}_{\mathrm{M}}=-\frac{1}{V(\omega)}\left[\begin{array}{ccc}
\mathbf{0}_{3 \times 9} & \boldsymbol{I}_{3 \times 3} & \mathbf{0}_{3 \times 1}
\end{array}\right]\left\{\boldsymbol{S}_{\varphi_{c}}^{\mathrm{T}} \frac{\partial \boldsymbol{\lambda}_{u_{c}}}{\partial \boldsymbol{K}_{\mathrm{M}}}+\boldsymbol{S}_{\varphi_{b}}^{\mathrm{T}} \frac{\partial \boldsymbol{\lambda}_{\varphi_{b}}}{\partial \boldsymbol{K}_{\mathrm{M}}}\right\}\left[\begin{array}{c}
\mathbf{0}_{9 \times 3} \\
\boldsymbol{I}_{3 \times 3} \\
\mathbf{0}_{1 \times 3}
\end{array}\right],
$$

and the apparent thermal expansion tensor $\boldsymbol{\alpha}_{\mathrm{M}}$ in the vector form using $-\boldsymbol{C}_{\mathrm{M}} \boldsymbol{A}_{\mathrm{M}}=\frac{\partial \boldsymbol{\Sigma}_{\mathrm{M}}}{\partial \vartheta_{\mathrm{M}}}$ following

$$
-\boldsymbol{C}_{\mathrm{M}} \boldsymbol{A}_{\mathrm{M}}=\frac{1}{V(\omega)}\left[\begin{array}{lll}
\boldsymbol{I}_{9 \times 9} & \mathbf{0}_{9 \times 3} & \mathbf{0}_{9 \times 1}
\end{array}\right]\left\{\boldsymbol{S}_{\varphi_{c}^{\mathrm{T}}} \frac{\partial \boldsymbol{\lambda}_{u_{c}}}{\partial \boldsymbol{K}_{\mathrm{M}}}+\boldsymbol{S}_{\varphi_{b}}^{\mathrm{T}} \frac{\partial \boldsymbol{\lambda}_{\varphi_{b}}}{\partial \boldsymbol{K}_{\mathrm{M}}}\right\}\left[\begin{array}{c}
\mathbf{0}_{9 \times 1} \\
\mathbf{0}_{3 \times 1} \\
\boldsymbol{I}_{1 \times 1}
\end{array}\right] .
$$




\subsection{Random field description of meso-scale material properties}

We have discussed how the apparent material tensor can be evaluated from finite element discretizations of the SVEs. Then, we can consider different SVE realizations, from which the distributions of the apparent meso-scale material tensors and their spatial correlations can be extracted. The detailed process to extract the random field description of the meso-scale material properties has been presented in [22] in the case of linear elasticity. This process is now extended to the thermo-mechanical random fields (24).

\subsubsection{SVE and random field realizations}

The purpose of this work is to evaluate the random field (24) of the meso-scale material tensors. To this end, a sufficient number of random field realizations is required, and each of these random field realizations is built up by a series of ordered SVE realizations obeying to a specified spatial relation. Taking the 3D case as an example, to obtain one random field realization, a volume which contains the information of the micro structure is first created. This volume should be large enough in order to be able to capture a complete spatial correlation, i.e. enough for all the spatial correlations to reach zero. Then, from this volume, a complete series of SVEs, which are indexed by the coordinates $\left(x_{1}, x_{2}, x_{3}\right)$ of their centers, is extracted, see Fig. 1(a). After evaluating the apparent meso-scale material properties on these SVEs, together with the spatial relation (the central coordinates) of these SVEs, a realization of the required random field is obtained. We need to note that the distance between the neighboring SVEs, which is defined by the distance between their centers, needs to be small enough to obtain the descriptions for the decline process of the spatial correlation functions.

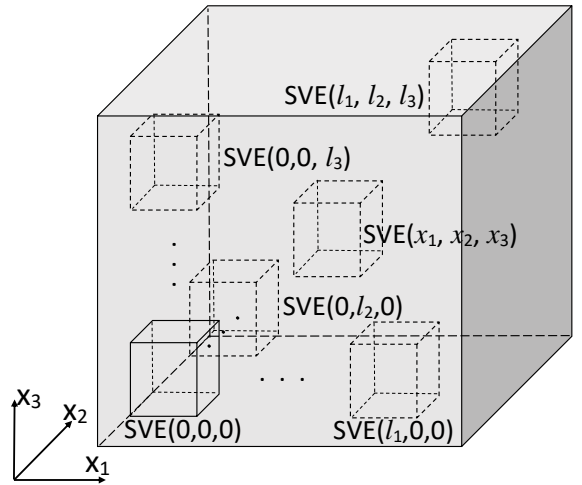

(a) 3-Dimensional case

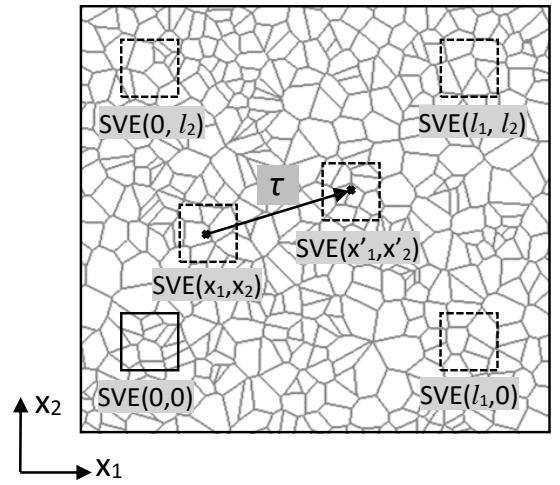

(b) 2-Dimensional case (Poisson-Voronoï tessellation)

Figure 1: Extraction of a series of SVEs

According to the discussion in Section 2.2, the required random field is the random vector field $\boldsymbol{V}(\boldsymbol{x}, \boldsymbol{\theta})$ (24), which contains the information of the elastic tensor $\boldsymbol{C}_{\mathrm{M}}$, thermal conductivity tensor $\boldsymbol{\kappa}_{\mathrm{M}}$, and thermal 
expansion tensor $\boldsymbol{\alpha}_{M}$ at the meso-scale. With a sufficient number of random field realizations, the spatial cross-correlation matrix $\boldsymbol{R}_{V}(\boldsymbol{x}, \boldsymbol{\tau})$, of the field $\boldsymbol{V}(\boldsymbol{x}, \boldsymbol{\theta})$ of the apparent meso-scale material properties, is evaluated as follows

$$
R_{V}^{(r s)}(\boldsymbol{x}, \boldsymbol{\tau})=\frac{\mathbb{E}\left[\left(V^{(r)}(\boldsymbol{x})-\mathbb{E}\left[V^{(r)}(\boldsymbol{x})\right]\right)\left(V^{(s)}(\boldsymbol{x}+\boldsymbol{\tau})-\mathbb{E}\left[V^{(s)}(\boldsymbol{x}+\boldsymbol{\tau})\right]\right)\right]}{\sigma_{V^{(r)}}(\boldsymbol{x}) \sigma_{V^{(s)}}(\boldsymbol{x}+\boldsymbol{\tau})} \quad \forall r, s=1, \ldots, 33,
$$

where $\boldsymbol{\tau}$ is a spatial vector between the centers of two SVEs, see Fig. 1(b), $V^{(r)}(\boldsymbol{x})$ is the $r^{\text {th }}$ element out of the 33 relevant elements of the material property vector $\boldsymbol{V}$ evaluated on the SVE with the center position $\boldsymbol{x}, \mathbb{E}$ is the expectation operator, and where $\sigma_{V^{(r)}}=\sqrt{\mathbb{E}\left[\left(V^{(r)}-\mathbb{E}\left[V^{(r)}\right]\right)^{2}\right]}$ is the standard deviation of $V^{(r)}$. The requirement of a large enough volume can be expressed as $R_{V}^{(r s)}(\boldsymbol{l})=0, \forall r, s=1, \ldots, 33$, see Fig. 1. In this work, we assume that the micro-structure distribution does not change with the position $\boldsymbol{x}$, i.e. the material structure is not graded. As a result, the random field is homogeneous and the cross-correlation matrix does not depend on the position $\boldsymbol{x}: \boldsymbol{R}_{V}(\boldsymbol{x}, \boldsymbol{\tau})=\boldsymbol{R}_{V}(\boldsymbol{\tau})$.

Poisson-Voronoï tessellations are generated to represent a columnar polycrystalline material, as illustrated in Fig. 1(b). The grain structures obtained by the Low Pressure Chemical Vapor Deposition (LPCVD) process are not strictly columnar. Indeed, small grains form at the lower side of the deposition, and while some of them stop growing, the remaining ones keep growing toward the top surface and dominate along the thickness. We thus assume in this work that a columnar structure represents the MEMS behavior. In this columnar structure, each grain possesses a different orientation, which is a source of uncertainty besides the grain geometry. As columnar SVEs are considered, the random field of poly-silicon layers is reduced to a 2D case, and the following procedure is considered. For a given SVE size, several SVEs of the same size can be extracted from a tessellation following the technique illustrated in Fig. 1. The detailed operations to extract the SVEs from a Poisson-Voronoï tessellation were discussed in [22, Appendix A]. A sufficient number of large Poisson-Voronoï tessellations is required for the description of the random field to converge.

As it has been discussed in [22], as a homogeneous meso-scale random field is assumed, a set of tessellation realizations -such a realization is illustrated in Fig. 1(b) - is enough to characterize the meso-scale random field of the whole macro-structure, and the size of the tessellations is not related to the size of the macrostructure.

\subsubsection{Random field generator}

Once the meso-scale random field is characterized using the realizations of the micro-meso homogenization, a random field generator can be built to provide as many realizations as required for the macro-scale SFEM analyzes. As generating new realizations using the generator is much less computationally costly than solving the SVE finite-element models, this approach is more computationally efficient to study different structural probabilistic problems.

In order for the random field generator to provide physically meaningful random material tensors, some 
pre-processes of the original data are carried out to ensure that the generated $\boldsymbol{C}_{\mathrm{M}}$ and $\boldsymbol{\kappa}_{\mathrm{M}}$ tensors remain positive definite, and that the expectations of their inverse exist. Therefore, respective lower bounds $\boldsymbol{C}_{\mathrm{L}}$ and $\boldsymbol{\kappa}_{\mathrm{L}}$ of those tensors are explicitly enforced ${ }^{3}$. The way of defining the lower bounds $\boldsymbol{C}_{\mathrm{L}}$ and $\boldsymbol{\kappa}_{\mathrm{L}}$ depends on the nature of the problem under consideration (inclusion reinforced-matrix, polycrystalline materials...) and is presented in Section 4 in the case of poly-silicon materials. Depending on the heterogeneous material system, some constrains can also be applied on thermal expansion tensor $\boldsymbol{\alpha}_{\mathrm{M}}$. For positive/negative thermal expansion material, the diagonal entries of $\boldsymbol{\alpha}_{\mathrm{M}}$ need to be positive/negative, or the trace of $\boldsymbol{\alpha}_{\mathrm{M}}$ need to be positive/negative. The constrain on thermal expansion tensors is out of the scope of this work, and the generation of this tensor will be discussed in Section 4.2.3 for the application of poly-silicon.

By introducing the lower bounds, $\boldsymbol{C}_{\mathrm{L}}$ and $\boldsymbol{\kappa}_{\mathrm{L}}$, the realizations of the random elasticity tensor and thermal conductivity tensor can be rewritten as

$$
\begin{gathered}
C_{\mathrm{M}}=C_{\mathrm{L}}+\Delta \boldsymbol{C}, \\
\kappa_{\mathrm{M}}=\kappa_{\mathrm{L}}+\Delta \boldsymbol{\kappa},
\end{gathered}
$$

where $\Delta \boldsymbol{C}$ and $\Delta \boldsymbol{\kappa}$ are positive definite matrices [22]. The Cholesky decomposition algorithm [44] can be used directly to obtain the positive definite matrices, which are expressed as

$$
\begin{aligned}
\Delta \boldsymbol{C} & =\mathcal{A A}^{\mathrm{T}}, \\
\Delta \boldsymbol{\kappa} & =\mathcal{B B}^{\mathrm{T}},
\end{aligned}
$$

where $\mathcal{A}$ and $\mathcal{B}$ are $6 \times 6$ and $3 \times 3$ lower triangular matrices, respectively, and superscript ' $\mathrm{T}$ ' refers to their transpose.

For each large micro structure realization, such as illustrated in Fig.1, we write the 21 entries of $\mathcal{A}, 6$ entries of $\boldsymbol{B}$ and 6 entries of the diagonal and symmetric part of $\boldsymbol{\alpha}_{\mathrm{M}}$ of each SVE into a vector $\mathcal{V}$ to obtain the realizations of the random vector field $\mathcal{V}(\boldsymbol{x}, \boldsymbol{\theta})$. Let $\overline{\mathcal{V}}$ and $\mathcal{V}^{\prime}$ be respectively the mean and fluctuation of the random vector field, with $\mathcal{V}=\overline{\mathcal{V}}+\mathcal{V}^{\prime}$. We assume that the random vector field $\mathcal{V}$ can be described as a homogeneous random field. Therefore $\overline{\mathcal{V}}$ is constant with respect to $\boldsymbol{x}$ and $\mathcal{V}^{\prime}$ is a homogeneous zero-mean random field. This required random field $\mathcal{V}^{\prime}(\boldsymbol{x}, \boldsymbol{\theta})$ can be generated through the spectral representation method suggested in [38] and based on the known cross-correlation matrix $\boldsymbol{R}_{\mathcal{V}^{\prime}}(\boldsymbol{\tau})$ (68), see Section 3.2.1. Finally, samples of meso-scale material properties can be obtained from Eqs. (69-72) as

$$
\begin{aligned}
\boldsymbol{C}_{\mathrm{M}}(\boldsymbol{x}, \boldsymbol{\theta}) & =\boldsymbol{C}_{\mathrm{L}}+\left(\overline{\mathcal{A}}+\mathcal{A}^{\prime}(\boldsymbol{x}, \boldsymbol{\theta})\right)\left(\overline{\mathcal{A}}+\mathcal{A}^{\prime}(\boldsymbol{x}, \boldsymbol{\theta})\right)^{\mathrm{T}} \\
\boldsymbol{\kappa}_{\mathrm{M}}(\boldsymbol{x}, \boldsymbol{\theta}) & =\boldsymbol{\kappa}_{\mathrm{L}}+\left(\overline{\mathcal{B}}+\mathcal{B}^{\prime}(\boldsymbol{x}, \boldsymbol{\theta})\right)\left(\overline{\mathcal{B}}+\mathcal{B}^{\prime}(\boldsymbol{x}, \boldsymbol{\theta})\right)^{\mathrm{T}}
\end{aligned}
$$

\footnotetext{
${ }^{3}$ A lower (upper) bound $\boldsymbol{A}_{\mathrm{L}}\left(\boldsymbol{A}_{\mathrm{U}}\right)$ of a matrix $\boldsymbol{A}$ is such that $\boldsymbol{u}^{\mathrm{T}}\left(\boldsymbol{A}-\boldsymbol{A}_{\mathrm{L}}\right) \boldsymbol{u} \geqslant 0\left(\boldsymbol{u}^{\mathrm{T}}\left(\boldsymbol{A}_{\mathrm{U}}-\boldsymbol{A}\right) \boldsymbol{u} \geqslant 0\right)$ for any vector $\boldsymbol{u} \in \mathbb{R}^{n}$, with $n=6$ and where we have used the Voigt notations for $\boldsymbol{A}$ representing the elastic operator $\boldsymbol{C}_{\mathrm{M}}$, and with $n=3$ for $\boldsymbol{A}$ representing the thermal conductivity tensor $\kappa_{\mathrm{M}}$.
} 
and

$$
\alpha_{\mathrm{M} i j}(\boldsymbol{x}, \boldsymbol{\theta})=\overline{\mathcal{V}}^{(t)}+\mathcal{V}^{(t)}(\boldsymbol{x}, \boldsymbol{\theta})
$$

where $i, j=1,2,3$ and $\mathcal{V}^{(t)}$ are the entries related to the thermal expansion component $\alpha_{\mathrm{M} i j}$.

In the case of poly-silicon layers with preferred grain orientations, the marginal distribution of $\mathcal{V}^{\prime(r)}$ obtained from the micro-sampling process, i.e. from the computation homogenization applied on the SVEs, turns out to be non-Gaussian and highly skewed. The spectral representation method developed in [38] is not directly applicable to generate non-Gaussian fields, and an iterative mapping process is thus required as suggested in $[45,34,5]$. In this iterative mapping process, the target spectrum $\boldsymbol{S}_{\text {Target }}^{(r s)}(\boldsymbol{\omega})$ is obtained through the Fourier transform of the auto/cross-correlation $R_{\mathcal{V}^{\prime}}^{(r s)}(\boldsymbol{\tau})$, where $r, s=1,2, \ldots, n$ are the indices of the entries in the random vector $\mathcal{V}^{\prime}$, according to Wiener-Khintchine relationship. A Gaussian stochastic vector field is first generated using the spectral representation method [38] and then mapped to a non-Gaussian field. Afterward, the spectrum of the resulting non-Gaussian field $\boldsymbol{S}_{\mathrm{NG}}^{(r s)}(\boldsymbol{\omega})$ is calculated and compared with the target spectrum $\boldsymbol{S}_{\text {Target }}^{(r s)}(\boldsymbol{\omega})$; if they are different, the spectrum $\boldsymbol{S}_{\mathrm{G}}^{(r s)}(\boldsymbol{\omega})$ used to generate the Gaussian field is corrected by the ratios between the entries of the spectrum of the resulting non-Gaussian field $\boldsymbol{S}_{\mathrm{NG}}^{(r s)}(\boldsymbol{\omega})$ and of the target spectrum $\boldsymbol{S}_{\text {Target }}^{(r s)}(\boldsymbol{\omega})$. This updated value of the spectrum $\boldsymbol{S}_{\mathrm{G}}^{(r s)}(\boldsymbol{\omega})$ is used in the next iteration to generate a new Gaussian field. Iterations are performed until $\boldsymbol{S}_{\mathrm{NG}}^{(r s)}(\boldsymbol{\omega}) \approx \boldsymbol{S}_{\text {Target }}^{(r s)}(\boldsymbol{\omega})$. The flow chart of this method is shown in Fig. 2, and the process is detailed in Appendix D.

\section{The micro-scale model}

Micro-structure uncertainties of polycrystalline materials, such as grain size and grain orientations, may result in a scatter of their mechanical properties, thermal conductivity, and thermal expansion. With a view toward the study of a MEMS resonator, we consider micro-structures made of silicon organized in a polycrystalline structure. Silicon is one of the most common material present in MEMS. Normally, the columnar polycrystalline material is obtained by LPCVD, and its micro structure, such as grain orientation and size, varies with the deposition temperature and time.

\subsection{The micro-structure characteristics of poly-silicon in MEMS}

The characteristic of poly-silicon micro structures can be determined using the measurements reported in $[23]$.

\subsubsection{Grain size}

Scanning Electron Microscope (SEM) is a typical measurement device used to characterize MEMS. For the deposition thickness of $2.0 \mu \mathrm{m}$ obtained at different deposition temperatures [23], the average grain sizes in terms of the diameter $\bar{d}$ corresponding to the SEM measurements are reported in Table 1. 


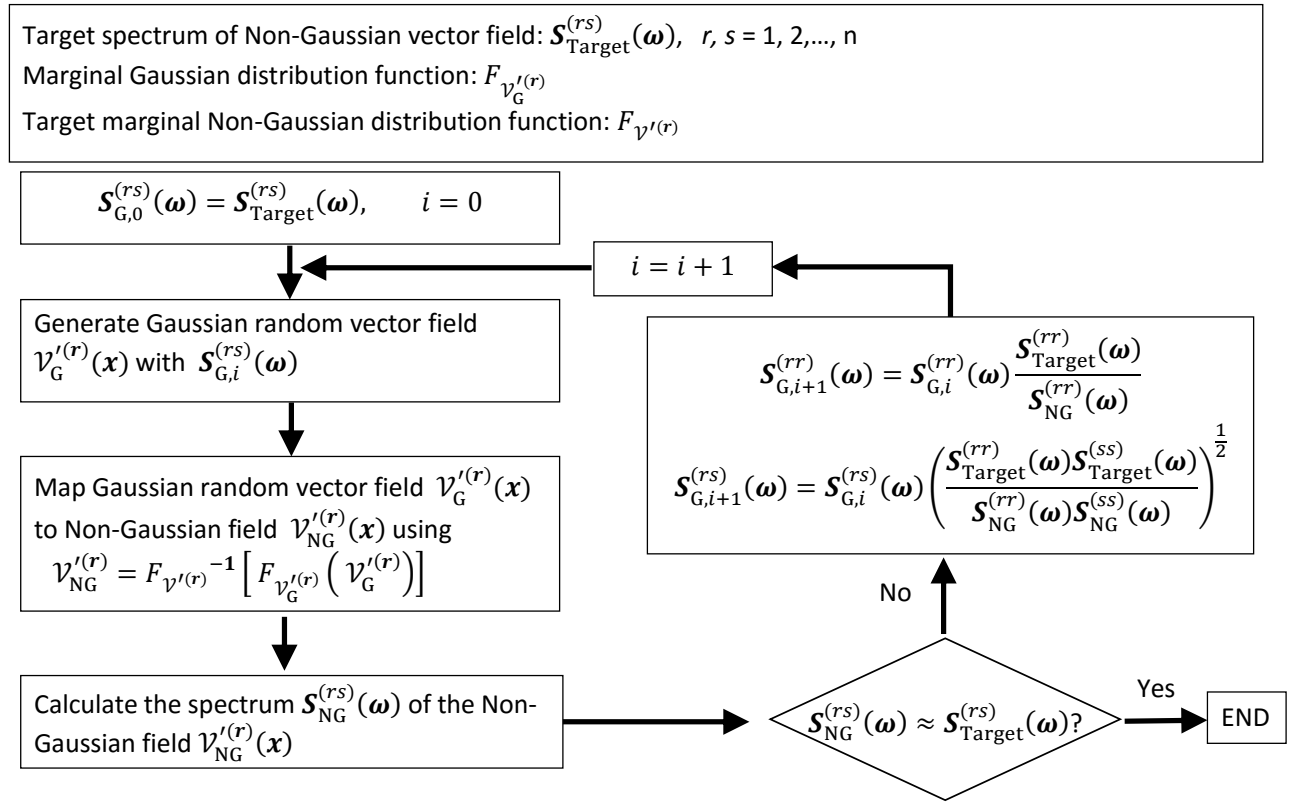

Figure 2: Flow chart of the non-Gaussian stochastic vector field generation

Table 1: Average grain sizes for different LPCVD temperatures [23]

\begin{tabular}{|c|c|c|c|c|}
\hline Temperature & $580^{\circ} \mathrm{C}$ & $610^{\circ} \mathrm{C}$ & $630^{\circ} \mathrm{C}$ & $650^{\circ} \mathrm{C}$ \\
\hline Average grain diameter $\bar{d}(\mu \mathrm{m})$ & 0.210 & 0.447 & 0.720 & 0.830 \\
\hline
\end{tabular}

\subsubsection{Grain orientations}

In order to simplify the modeling of the micro structure in this work, we assume that the crystallinity is $100 \%$. The grain orientations in poly-silicon can be measured by X-ray Diffraction (XRD). According to the XRD measurements on $2.0 \mu \mathrm{m}$-thick poly-silicon layers [23], the different orientations of the grains and their respective volume ratio are presented in the Table 2 for different deposition temperatures. The different directions corresponding to the normal to the surface of the sample are indicated with respect to the local crystal basis using Miller indexes.

\subsection{The physical properties of poly-silicon in MEMS}

The mechanical properties, thermal conductivity tensors, and thermal expansion tensors of the silicon grains are discussed and presented in this section, which allow defining the corresponding bounds for the scattering tensors either by using theoretical computation or physically based assumptions. 
Table 2: The percentages of grains in the different orientations for different LPCVD temperatures (\%) [23]

\begin{tabular}{|c|c|c|c|c|}
\hline Orientation & $580^{\circ} \mathrm{C}$ & $610^{\circ} \mathrm{C}$ & $630^{\circ} \mathrm{C}$ & $650^{\circ} \mathrm{C}$ \\
\hline$<111>$ & 12.57 & 19.96 & 12.88 & 11.72 \\
\hline$<220>$ & 7.19 & 13.67 & 7.96 & 7.59 \\
\hline$<311>$ & 42.83 & 28.83 & 39.08 & 38.47 \\
\hline$<400>$ & 4.28 & 5.54 & 3.13 & 3.93 \\
\hline$<331>$ & 17.97 & 18.14 & 21.32 & 20.45 \\
\hline$<422>$ & 15.16 & 13.86 & 15.63 & 17.84 \\
\hline
\end{tabular}

\subsubsection{Mechanical properties of a single silicon crystal}

Silicon material is anisotropic, with a cubic symmetry, and the properties of a silicon grain depend on its orientation with respect to the crystal lattice. For the silicon crystal oriented with [100], [010] and [001] along the Cartesian coordinates [14], the crystal elasticity tensor $\boldsymbol{C}_{\mathrm{S}}$ reads, in GPa,

$$
\boldsymbol{C}_{\mathrm{S}}=\left[\begin{array}{cccccc}
165.7 & 63.9 & 63.9 & 0 & 0 & 0 \\
63.9 & 165.7 & 63.9 & 0 & 0 & 0 \\
63.9 & 63.9 & 165.7 & 0 & 0 & 0 \\
0 & 0 & 0 & 79.6 & 0 & 0 \\
0 & 0 & 0 & 0 & 79.6 & 0 \\
0 & 0 & 0 & 0 & 0 & 79.6
\end{array}\right]
$$

Based on the elasticity tensor $\boldsymbol{C}_{\mathrm{S}}$ of the silicon crystal, the elasticity tensors of poly-silicon SVEs, $\boldsymbol{C}_{\mathrm{M}}$, can be determined following the homogenization process presented in Section 3.1. The methodology to determine the lower bound $\boldsymbol{C}_{\mathrm{L}}$ required by the generator, see Section 3.2.2, was developed in [22]: the lower bound $\boldsymbol{C}_{\mathrm{L}}$ for the polycrystalline material is defined as the isotropic tensor $\boldsymbol{C}^{\text {iso }}$ solution of

$$
\begin{array}{r}
\min _{E, \nu \in R^{+}}\left\|\boldsymbol{C}^{\text {iso }}-\boldsymbol{C}_{\mathrm{S}}^{\mathrm{R}}\right\| \quad \text { subject to } \boldsymbol{u}^{\mathrm{T}}\left(\boldsymbol{C}_{\mathrm{S}}^{\mathrm{R}}-\boldsymbol{C}^{\text {iso }}\right) \boldsymbol{u} \geqslant 0 \text { for any vector } \boldsymbol{u} \in \mathbb{R}^{6} \\
\text { and for any rotation } \boldsymbol{C}_{\mathrm{S}}^{\mathrm{R}} \text { of } \boldsymbol{C}_{\mathrm{S}},
\end{array}
$$

where $E$ is the Young's modulus and $\nu$ the Poisson coefficient of the isotropic material. The lower bound is thus obtained by solving the optimization problem (77) leading to $\boldsymbol{C}_{\mathrm{L}}=\boldsymbol{C}^{\text {iso }}(E=130.0 \mathrm{GPa}, \nu=0.278)$.

\subsubsection{Thermal conductivity tensor of poly-silicon}

The thermal conductivities for thin films of poly-silicon are substantially different from those of the bulk silicon. Depending on the applications of MEMS, different deposition and doping techniques, which affect the micro structure of poly-silicon, will be adopted and different thermal conductivities of the resulting 
materials are observed. The thermal conductivities of poly-silicon layers depend on the grain size and shape, and the concentration and type of dopant atoms as shown in [29].

In dielectric materials, heat transport in both amorphous and crystalline solids occurs by elastic vibrations of the lattice; phonons are the energy quanta of lattice vibration waves and are the main energy carriers. This transport mode is limited by the elastic scattering of phonons due to the lattice defects. The effect of boundary scattering of phonons on thermal conductivities in single-crystal silicon layers have been previously investigated in [2], and the thermal conductivities of poly-silicon are found to be lower than those of single-crystal silicon layers due to phonon scattering at the grain boundaries. For doped silicon layers, such as phosphorus and boron-doped silicon layers, with impurity concentrations, the impurity scattering causes a further reduction in the thermal conductivity as compared to pure silicon layers. Because of the difficulties in the modeling of phonon scattering at grain boundaries, the theoretical prediction of the thermal conductivity for a given impurity concentration and micro structure is still not possible [29].

Based on theoretical models and the experimental measurements, the thermal conductivity of poly-silicon layer was given in a simplified closed-form expression in [29]. This closed-form expression which relates the room-temperature thermal conductivity to the grain size and to the impurity concentration and type, reads

$$
\kappa\left(\bar{d}, c_{\mathrm{i}}\right)=\frac{1}{3} C_{\mathrm{S}} v_{\mathrm{s}}\left(\frac{A_{1}}{\bar{d}}+A_{2} c_{\mathrm{i}}\right)^{-1},
$$

in which the obtained thermal conductivity has the unit of $\mathrm{W} /(\mathrm{m} \cdot \mathrm{K}), \bar{d}$ is the average grain size in unit of $\mathrm{nm}, c_{\mathrm{i}}$ is the impurity concentration in unit of $\mathrm{cm}^{-3}, v_{\mathrm{s}}=6166 \mathrm{~m} / \mathrm{s}$ is the averaged phonon group velocity in silicon, and $C_{\mathrm{S}}=1.654 \times 10^{6} \mathrm{~J} / \mathrm{m}^{3} \mathrm{~K}$ is the phonon specific heat at $300 \mathrm{~K}$ in silicon. The constants $A_{1}$ and $A_{2}$ depend on the dopant atoms, which are known from the fabrication process. In this work, we assume that the poly-silicon is boron-doped, which gives $A_{1}=2.887 \times 10^{10}$ and $A_{2}=3.200 \times 10^{-13} \mathrm{~m}^{2}$, and a measured impurity concentration $c_{\mathrm{i}}=1.6 \times 10^{19} \mathrm{~cm}^{-3}$ is chosen for our applications.

The conductivities obtained from Eq. (78) are related to the average grain size and take into account the grain boundaries present along the 3 directions (in-plane and out-of-plane directions of the poly-silicon layer). As using these results in the meso-scale (SVEs) simulations, in which only few grains exist in each SVE, is not feasible, we assume that Eq. (78) also holds for only one grain with the effect of its boundaries included. Hence, because of the cubic symmetric of silicon crystal, a thermal conductivity and an isotropic thermal conductivity tensor are computed for this grain, depending on the size of a grain, which results in

$$
\kappa_{\mathrm{m} i j}(\bar{d})=\delta_{i j} \kappa(\bar{d})
$$

where $\delta_{i j}$ is the Kronecker symbol. We need to note that it would also be possible to define an anisotropic thermal conductivity tensor according to the grain's geometry which includes the information of the grain shape and boundaries. However, in this work, we consider an isotropic thermal conductivity tensor for each grain to keep the problem simple. 
Since the apparent thermal conductivity tensors of SVEs are combined results of a few grains, it is possible to define a low/high bound from Eq. (78) by using a grain size much smaller/higher than the generated grain size of the considered poly-silicon SVEs. For example, when we consider the poly-silicon layer deposited at the temperature of $610^{\circ} \mathrm{C}$, the average grain size $d=210 \mathrm{~nm}$ at the deposition temperature of $580^{\circ}$, see Table 1 , can be used to define a low bound for the thermal conductivities of SVEs, and one has

$$
\kappa_{\mathrm{SVE}}>\kappa_{\mathrm{L}}, \quad \text { and } \quad \kappa_{\mathrm{L}}=\kappa(210 \mathrm{~nm})
$$

The lower bound defined in this way is shown to be valid, for the SVEs samples of the poly-silicon layer deposited at the temperature of $610^{\circ} \mathrm{C}$, in Section 5 .

\subsubsection{Thermal expansion tensor of a single silicon crystal}

The thermal expansion tensor is a structure sensitive property and reflects any change in the micro structure. For crystalline materials, the number of independent thermal expansion components varies with the type of crystal system [7]. The tensor for polycrystalline materials is more complicated and has a wide divergence. However the thermal expansion tensors of different grains differ only by their orientation.

For cubic symmetric crystal systems, the thermal expansion tensor has a simple form as $\boldsymbol{\alpha}_{i j}=\delta_{i j} \alpha_{T}$, where the linear thermal expansion coefficient, $\alpha$, at temperature $T$ expressed in $\mathrm{K}$, can be calculated from an empirical equation. Around $T_{\mathrm{R}}=273 \mathrm{~K}$ and above, this empirical equation for a wide range of temperatures is given by

$$
\alpha_{T}=A+B\left(T-T_{\mathrm{R}}\right)+C\left(T-T_{\mathrm{R}}\right)^{2}
$$

in terms of the coefficients $A, B$, and $C$, respectively expressed in $\mathrm{K}^{-1}, \mathrm{~K}^{-2}$, and $\mathrm{K}^{-3}$.

For silicon crystal, at $T_{\mathrm{R}}=273 \mathrm{~K}$ the different factors are identified as $A=3.084 \times 10^{-6} / \mathrm{K}(T: 293 \sim$ $970 \mathrm{~K})$ and $A=2.327 \times 10^{-6} / \mathrm{K}(T: 298 \sim 314 \mathrm{~K})$ according to different references, $B=1.957 \times 10^{-9} / \mathrm{K}^{2}$ and $C=0$ [7]. The value of $A=3.084 \times 10^{-6} / \mathrm{K}$ is adopted in this work.

Although in all generalities, the apparent thermal expansion tensor $\boldsymbol{\alpha}_{\mathrm{M}}$ of an SVE can be computed through equation (67) following the homogenization process described in Section 3.1, because of the cubic symmetry of the silicon crystal, the poly-silicon SVEs all have the same isotropic thermal expansion tensor

$$
\boldsymbol{\alpha}_{\mathrm{Mij}}=\delta_{i j} \alpha_{\mathrm{m}}
$$

where $\alpha_{\mathrm{m}}=3.084 \times 10^{-6}+1.957 \times 10^{-9}\left(T-T_{\mathrm{R}}\right)$ in $\mathrm{K}^{-1}$. In the conducted modal analyzes the equations are linearized and the material parameters are considered as constant with respect to the temperature $\left(T \simeq T_{0}\right)$.

\subsection{Generation of poly-silicon SVEs}

Considering poly-silicon layers fabricated through LPCVD at $610^{\circ} \mathrm{C}$ and $630^{\circ} \mathrm{C}$ as the references to create the micro structures, the average grain diameters $\bar{d}$ of $447 \mathrm{~nm}$ and $720 \mathrm{~nm}$ accordingly to the SEM 
measurements reported in Table 1, are respectively used to generate the Voronoï tessellations. Since the considered MEMS structures are fabricated through LPCVD, similar micro material structures (at the grain scale) will be obtained on the whole MEMS-structure, which justifies the use of a homogeneous random field.

The SVEs are extracted from the 2D Voronoï tessellations, see Fig. 1(b), and extruded into 3D under the assumption that the poly-silicon layers are columnar polycrystalline materials. The SVE size is selected according to the size of grains. In [22], it was shown that when using macro-scale SFEM, the results converge with respect to the macro-scale mesh size, for any size of the underlying SVEs, as long as the resulting correlation length of the meso-scale random field is larger than the distance between the integration points of the macro-scale SFEM. However, this implies for the meso-scale random field to be correctly defined. The SVEs thus need to include (parts of) a few grains so that the spatial correlation is correctly captured. Indeed, if the SVEs include only a part of a single grain the result is no longer a homogenized one, and the meso-scale field becomes a noise field [3], leading a non-smooth spatial correlation. The size of the SVEs is also upper bounded by the requirement of length-scale separation between the macro-scale and the meso-scale: the SVEs size should be one order lower than the macro-scale characteristic length. Moreover increasing the SVEs size increases the computational time required to define the meso-scale random field, although it allows using coarser SFEM at the macro-scale as the correlation length increases.

We chose the SVE sizes of $0.5 \mu \mathrm{m}$ and $0.8 \mu \mathrm{m}$ for the poly-silicon layers deposited at $610^{\circ} \mathrm{C}$ and $630^{\circ} \mathrm{C}$, respectively, and the examples of SVE finite element discretizations are given in Fig. 3. The SVEs contain parts of several grains and it will be shown in Section 5.2 that the extracted meso-scale random field spatial correlation remains smooth. The mesh size of the macro-scale SFEM will be chosen so that the distance between the integration points remains lower than the meso-scale random field correlation length, which results from this SVE size, as it will be discussed in Section 6 .

In order to build the meso-scale random fields using the window technique as illustrated in Fig. 1(b), 200 (140) large Voronoï tessellations are built for the poly-silicon layers deposited at $610^{\circ} \mathrm{C}\left(630^{\circ} \mathrm{C}\right.$, respectively), from which 100 SVEs are extracted per tessellation by progressing by a distance of $0.25 \mu \mathrm{m}(0.4 \mu \mathrm{m}$, respectively). The resulting number of SVEs, 20000 (140 000) SVEs for the poly-silicon layers deposited at $610^{\circ} \mathrm{C}\left(630^{\circ} \mathrm{C}\right.$, respectively), ensures the convergence of the extracted meso-scale random fields.

\section{Mesoscopic scale: elasticity tensor and heat conductivity tensor generation}

In this section, the elasticity tensor and heat conductivity tensor distributions, which are obtained directly from micro-sampling, i.e. from the homogenization on SVE realizations, are presented and compared with the ones obtained from the non-Gaussian random vector field generator. Compared to our previous work [22], the spectral generator is now combined to a non-Gaussian mapping in order to be able to generate random fields characterized by skewness and/or Kurtosis values different from Gaussian distributions. The 


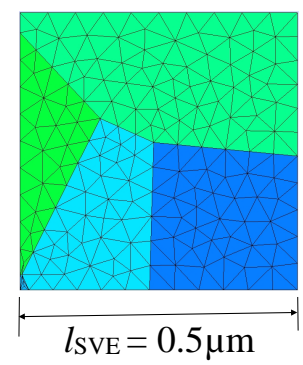

(a) LPCVD at $610^{\circ}$

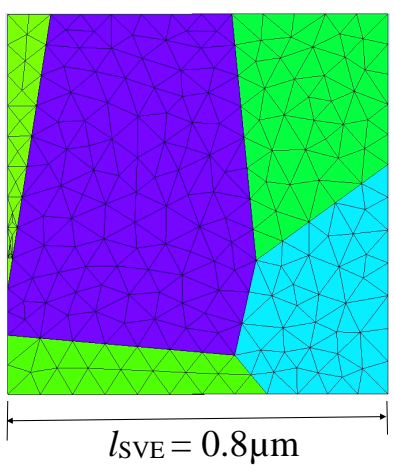

(b) LPCVD at $630^{\circ}$

Figure 3: Examples of SVE

purpose of this section is to discuss the accuracy of the resulting non-Gaussian random field generator, in particular for lowly, mildly, and highly correlated material parameters. For illustration purpose, only the results for the poly-silicon layers deposited at $610^{\circ} \mathrm{C}$ are presented here for comparison. In Section 6 , the elasticity tensors and heat conductivity tensors of the poly-silicon layers deposited at $630^{\circ} \mathrm{C}$ will also be used to predict the thermo-elastic quality factor distribution of MEMS resonators to reveal the effect of fabrication on the structural response.

\subsection{Random vector field of $\mathcal{V}^{\prime}$}

We consider the poly-silicon layers deposited at $610^{\circ} \mathrm{C}$ with the thickness of $2.0 \mu \mathrm{m}$. The average grain size is $447 \mathrm{~nm}$, and the SVE size of $0.5 \mu \mathrm{m}$ (see Fig. 3(a)) is used in the homogenization process. According to the homogenized results, the obtained thermal conductivity tensor has three different non-zero entries, $\kappa_{\mathrm{M} i i},(i=1,2,3)$, therefore, the random vector $\mathcal{V}^{\prime}$ has totally 24 entries with $\mathcal{V}^{(1)}, \ldots, \mathcal{V}^{(21)}$ relating to the random meso-scale elastic tensors $\boldsymbol{C}_{\mathrm{M}}$ and $\mathcal{V}^{(22)}, \mathcal{V}^{\prime(23)}$ and $\mathcal{V}^{\prime(24)}$ relating to the random meso-scale thermal conductivity tensors $\boldsymbol{\kappa}_{\mathrm{M}}$ (see Section 3.2.2).

Because a mapping process is used in the non-Gaussian generator, the distributions of the generated $\mathcal{V}^{\prime(r)},(r=1,2, \ldots, 24)$ recover the exact distributions of the micro-samples, which are obtained from the homogenization on SVE realizations. In Fig. 4, the histograms obtained by micro-sampling and by the generator are compared for $\mathcal{V}^{(6)}$ and $\mathcal{V}^{(9)}$, which have a highly skewed distributions. The generated random variables represent well their distributions obtained from the homogenization on SVEs.

Auto-correlation functions $R_{\mathcal{V}^{\prime}}^{(r r)}(r=1,2, \ldots, 24)$ are also well recovered by the non-Gaussian random vector generator, as illustrated by Fig. 5, which depicts the 3D-view of the normalized auto-correlation function $R_{\mathcal{V}^{\prime}}^{(1,1)}$. Since the 2-dimensional correlation functions are symmetric with respect to the planes $x=0$ and $y=0$, only one quarter of the function is displayed. More comparisons of auto-correlation curves, 


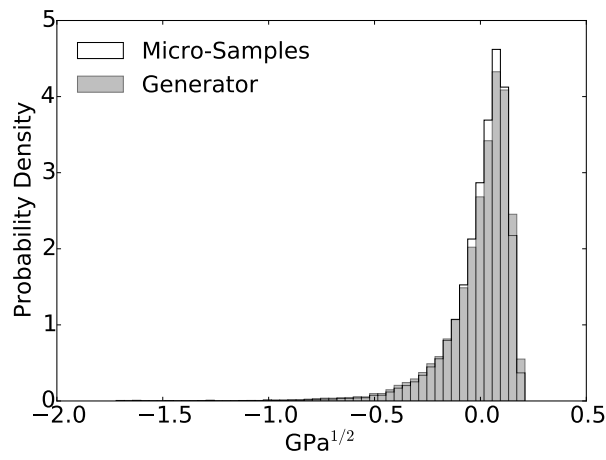

(a) Histogram of $\mathcal{V}^{(6)}$

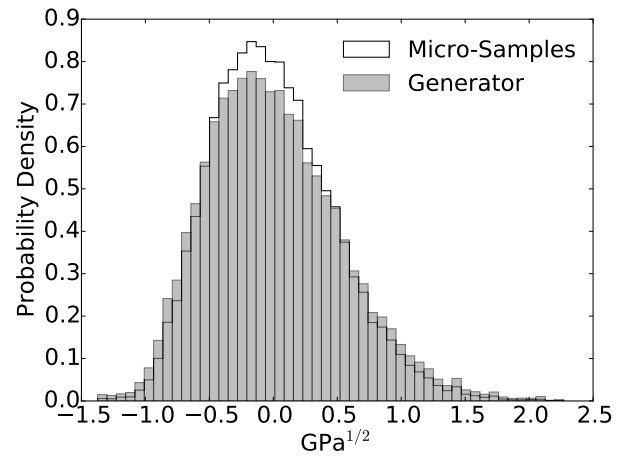

(b) Histogram of $\mathcal{V}^{(9)}$

Figure 4: Comparison of histograms of $\mathcal{V}^{\prime(r)}$ between the micro-samples and the generated samples

in the $y=0$-plane, are presented in Fig. 6, for which the entries of $\mathcal{V}^{\prime}$ are picked randomly from those related to the material tensor, Figs. 6(a)-6(c), and to the thermal conductivity tensor, Fig. 6(d).

For the cross-correlation functions, their trends are well preserved by the random field generator, as demonstrated by Fig. 7, which shows the 3D-view of the normalized cross-correlation function $R_{\mathcal{V}^{\prime}}^{(1,2)}$. More cross correlations are compared in Fig. 8. On the one hand, when the two random variables are not correlated, the cross-correlation $R_{\mathcal{V}^{\prime}}^{(r s)}=0$ is obtained for both micro-samples and generated samples, see Fig. 8(a) and Fig. 8(b) for the comparison of cross-correlation curves in the $y=0$-plane. On the other hand, when the two variables are highly correlated, the cross-correlations are accurately recovered by the non-Gaussian random vector field generator, see Fig. 8(c). However, when the two random variables are moderately correlated, the cross-correlations obtained by the generator are less accurate and only their trends are preserved, see Fig. 8(d).

\subsection{Random field of material properties $\boldsymbol{C}_{M}$ and $\boldsymbol{\kappa}_{M}$}

Using equations (73) and (74), the random field of the material properties $\boldsymbol{C}_{\mathrm{M}}(\boldsymbol{X}, \boldsymbol{\theta})$ and $\boldsymbol{\kappa}_{\mathrm{M}}(\boldsymbol{X}, \boldsymbol{\theta})$ can be computed for further probabilistic macro-scale structural analyses. The histograms of some entries of $\boldsymbol{C}_{\mathrm{M}}$ and $\boldsymbol{\kappa}_{\mathrm{M}}$, which are obtained from the generated $\mathcal{V}^{\prime}$, are presented in Fig. 9, in which the results from the micro-samples are also presented for comparison purpose. When using Eq. (73) to compute $\boldsymbol{C}_{\mathrm{M}}$, for different $C_{\mathrm{M} i j},(i, j=1,2, \ldots, 6)$, different numbers of entries of $\mathcal{V}^{\prime}$ will be used: for $C_{11}$ only $\mathcal{V}^{(1)}$ is used, but for $C_{\mathrm{M} 66}$, the entries from $\mathcal{V}^{\prime(16)}$ to $\mathcal{V}^{(21)}$ are required. As expected, for the components of $\boldsymbol{C}_{\mathrm{M}}$ and $\boldsymbol{\kappa}_{\mathrm{M}}$ computed using only one entry of $\mathcal{V}^{\prime}$, their distributions are well recovered by the generator, see Figs. 9(a) and 9(d). However, for the components of $\boldsymbol{C}_{\mathrm{M}}$ using more entries from $\mathcal{V}^{\prime}$, the distributions of their generated samples have more discrepancy with the distributions of their micro-samples, see Fig. 9(b). The worst case is found for $C_{\mathrm{M} 66}$, Fig. 9(c), in which the maximum number of entries $\mathcal{V}^{\prime(r)}$ is involved. This 


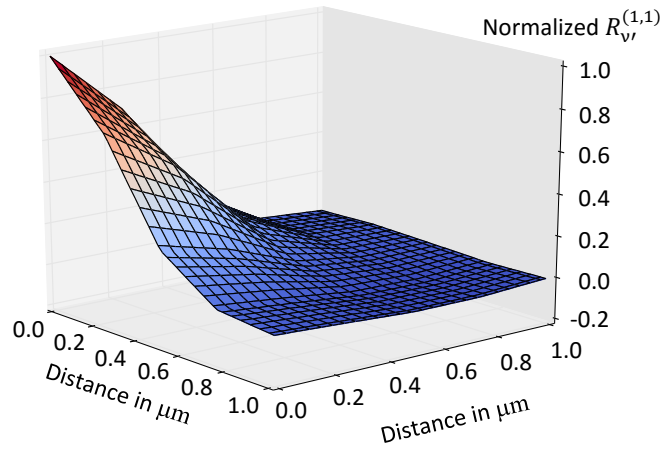

(a) Micro-samples

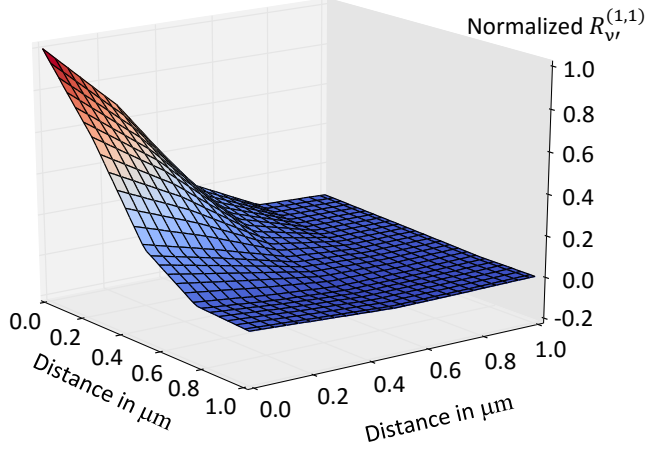

(b) Generator

Figure 5: Comparison of a normalized auto-correlation function between the micro-samples and the generated samples (3Dview)

problem can be explained by the reduced accuracy of the cross-correlation obtained by the non-Gaussian generator: when more random variables $\mathcal{V}^{\prime(r)}$ are used to compute a component of $\boldsymbol{C}_{\mathrm{M}}$, the effect of a loss of accuracy in the cross-correlation becomes more obvious.

Although the non-Gaussian random field generator gives a less accurate distribution for a few entries in $\boldsymbol{C}_{\mathrm{M}}$, those entries are related to the shearing behavior of the material, which is not an important property in our application, while the material properties related to the tension and compression, which are the most important properties to estimate the thermo-mechanical damping are accurately represented. However, this inaccuracy can lead to some physically unreasonable values for those entries. In order to keep all the entries in a physically admissible range, an extra mapping process can be applied on the entries with inaccurate distribution, following

$$
C_{\mathrm{M}_{i j}}^{\mathrm{C}}=F_{C_{\mathrm{M} i j}}{ }^{-1}\left[F_{C_{\mathrm{M}}^{\mathrm{NG}} i j}\left(C_{\mathrm{M} i j}\right)\right],
$$

where $C_{\mathrm{M} i j}$ and $C_{\mathrm{M}_{i j}}^{\mathrm{C}}$ are the generated components respectively without and with the mapping correction, $F_{C_{\mathrm{M} i j}}{ }^{-1}$ is the inverse marginal distribution function of the component $C_{\mathrm{M} i j}$ obtained from the microsamples and $F_{C_{\mathrm{M}}^{\mathrm{NG}} i j}$ the marginal distribution function of the component $C_{\mathrm{M} i j}$ obtained from the nonGaussian generator. In Fig. 10, the marginal distributions of $C_{\mathrm{M} 55}$ obtained from the generated samples are presented before (Fig. 10(a)) and after (Fig. 10(b)) the mapping correction. However, the proper solution to address the inaccurate shearing properties is to use a more accurate non-Gaussian vector field generator, which is out of the scope of this work.

Finally, some realizations of the random field are presented in Fig. 11 for the components picked as examples. For both the 2D view, Fig. 11(a) and Fig. 11(b), and the 3D view, Fig. 11(c) and Fig. 11(d), 


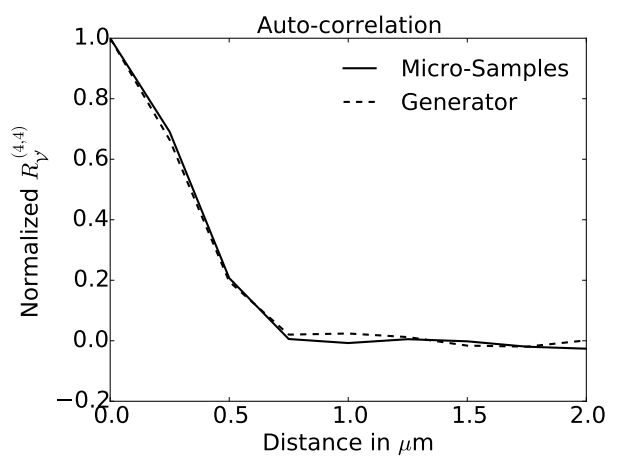

(a) $\mathcal{V}^{\prime(4)}$



(c) $\mathcal{V}^{\prime(19)}$

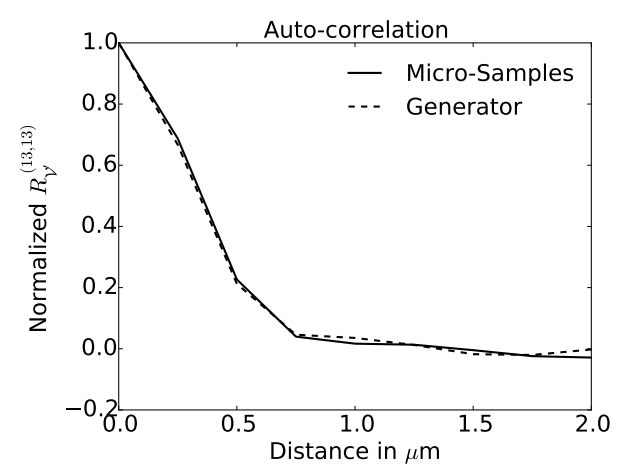

(b) $\mathcal{V}^{\prime(13)}$

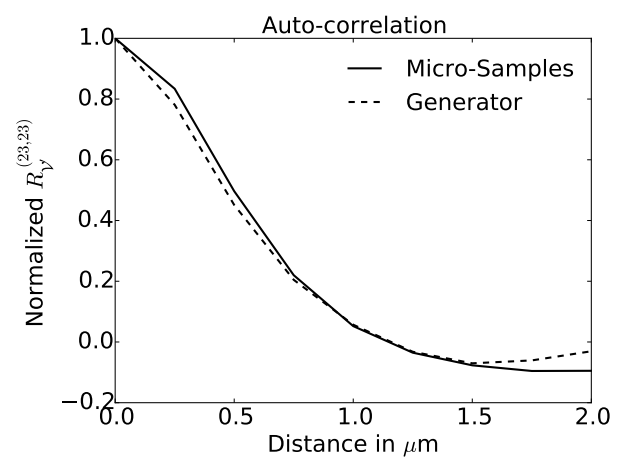

(d) $\mathcal{V}^{\prime(23)}$

Figure 6: Comparison of normalized auto-correlation functions between micro-sampling and generated samples in the $y=0$ plane (2D-view)

of the random field, a similar correlation of the material properties among the neighboring material points can be seen between the micro-samples and the generated ones.

\section{Macro-scale: stochastic study on the thermo-elastic quality factor of MEMS resonator}

In this section, we study the thermo-elastic quality factor of micro-resonators which are fabricated at two different temperatures, $610^{\circ} \mathrm{C}$ and $630^{\circ} \mathrm{C}$, respectively.

The Finite element analysis which is presented in Section 2 is applied on the micro-resonator. The micro-resonator is simplified as a micro-cantilever beam with the dimension of $L \times w \times t$, see Fig. 12. The material properties at each integration point are given by the random field generator as described in Section 5.2 , which is based on the stochastic homogenization of meso-scale SVEs presented in Section 4.3. The discretization of the random field is in agreement with the finite element discretization. However in order to obtain a converged stochastic finite element analysis, the spacing of the discretized random field, i.e. the 


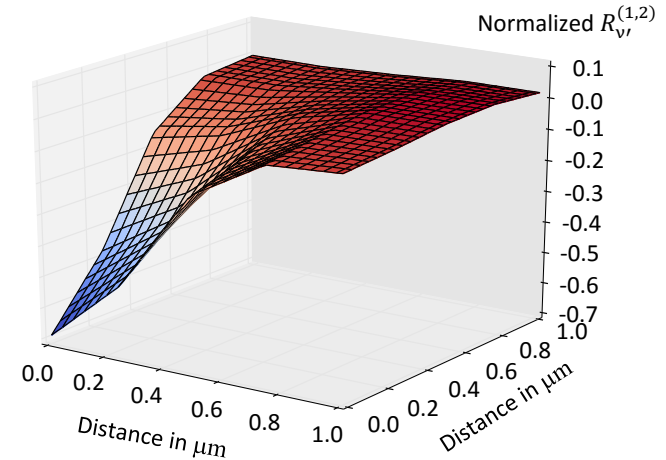

(a) Micro-Samples

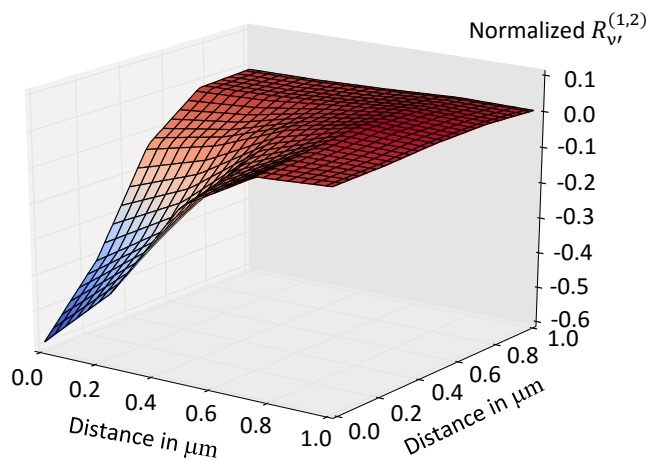

(b) Generator

Figure 7: Comparison of a normalized cross-correlation function between micro-samples and generated samples (3D-view)

spacing between the integration points, must be lower than the correlation length $l_{\mathbf{C}}$ of the random field, as this has been demonstrated by the convergence study performed in [22]. According to the homogenized results at the meso-scale, the correlation length of the random field of the material properties $\boldsymbol{C}_{\mathrm{M}}(\boldsymbol{x}, \boldsymbol{\theta})$ and $\boldsymbol{\kappa}_{\mathrm{M}}(\boldsymbol{x}, \boldsymbol{\theta})$ can be calculated by equation (26) and corresponds to $l_{\mathbf{C}}^{610} \approx 0.73 \mu \mathrm{m}$ for the poly-silicon layer deposited at $610^{\circ} \mathrm{C}$ and with the SVE size of $0.5 \mu \mathrm{m}$, and to $l_{\mathbf{C}}^{630} \approx 1.23 \mu \mathrm{m}$ for the poly-silicon layer deposited at $630^{\circ} \mathrm{C}$ and with the SVE size of $0.8 \mu \mathrm{m}$. In the conducted Finite Element analyses, we use quadratic elements with $3 \times 3 \times 3$ Gauss integration points. The largest finite elements that are considered in this section are of size $1 \times 1 \times 1 \mu \mathrm{m}^{3}$, leading to a maximum distance between the integration points of about $0.387 \mu \mathrm{m}$, which is smaller than the correlation length of the meso-scale random fields.

The generated random field is evaluated at the different positions of the Integration Gauss Points (IP) in order to define the SFEM problem. However care must be taken of when the integration points are close to the geometry boundary as the heterogeneities number can then be overestimated as the centered SVE has part of his domain outside of the macro-scale mesh as depicted in Fig. 13. To circumvent this issue, the associated SVE center is moved inward the domain to represent the correct amount of heterogeneities as described in Fig. 13. This issue can also be solved by using a different kind of random field discretization than the point discretization method.

Besides the random fields considered for the material properties $\boldsymbol{C}_{\mathrm{M}}(\boldsymbol{x}, \boldsymbol{\theta})$ and $\boldsymbol{\kappa}_{\mathrm{M}}(\boldsymbol{x}, \boldsymbol{\theta})$, the necessary deterministic properties for the analyses are the density $\rho$, the thermal expansion coefficient $\alpha$, and the heat capacity per unit mass at constant volume $C_{v}$ for poly-silicon. The adopted values in this work are

$$
\rho_{\mathrm{M}}=2330 \mathrm{Kg} / \mathrm{m}^{3}, \quad \alpha_{\mathrm{M}}=3.084^{-6} / \mathrm{K} \quad \text { and } \quad C_{v \mathrm{M}}=712 \mathrm{~J} /(\mathrm{Kg} \cdot \mathrm{K}) .
$$




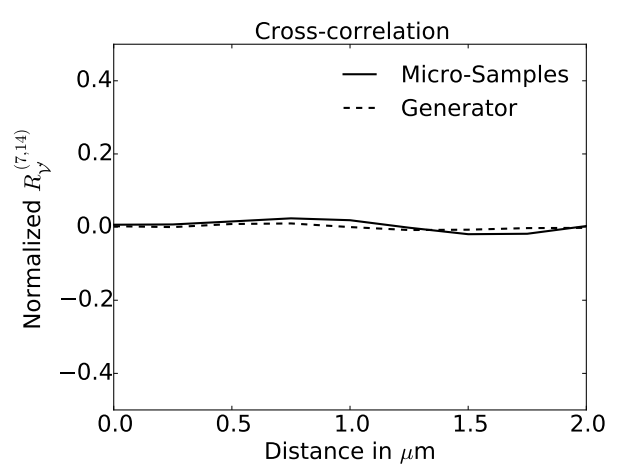

(a) $\mathcal{V}^{(7)}$ and $\mathcal{V}^{\prime(14)}$

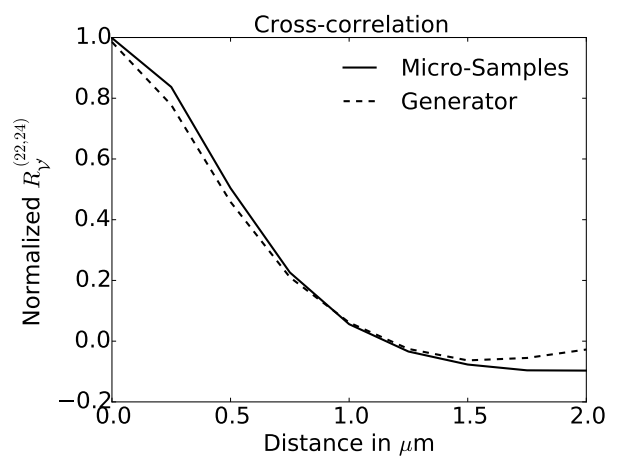

(c) $\mathcal{V}^{\prime(22)}$ and $\mathcal{V}^{\prime(24)}$

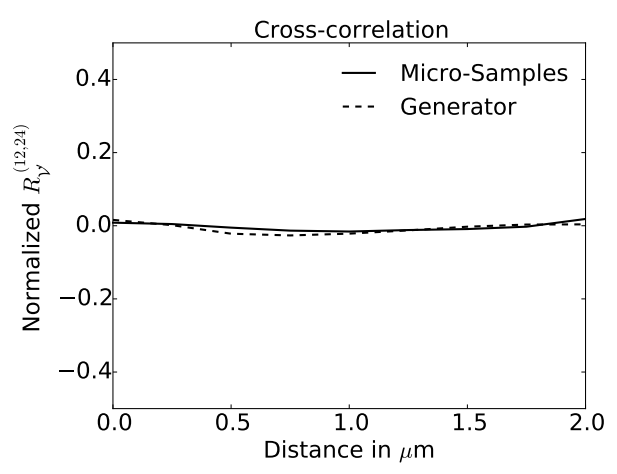

(b) $\mathcal{V}^{(12)}$ and $\mathcal{V}^{(24)}$

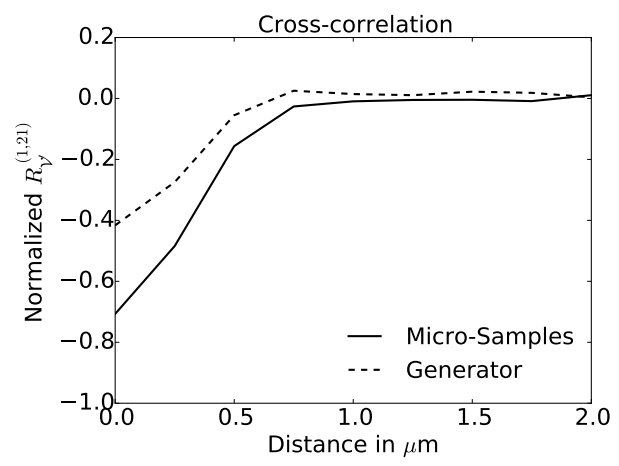

(d) $\mathcal{V}^{\prime(1)}$ and $\mathcal{V}^{\prime(21)}$

Figure 8: Comparison of normalized cross-correlation functions between micro-samples and generated samples in plane $y=0$ (2D-view)

\subsection{Idealized cantilever}

First a clamped cantilever beam is studied following the schematics of Fig. 12(a). At clamp, the boundary conditions read

$$
\boldsymbol{u}=0 \quad \text { and } T=T_{0} \text {. }
$$

We consider different beam geometries and different deposition temperature cases following the notation " $L \times w \times t, T_{\text {depos }}$ ", where $L, w$, and $t$ are the beam length, width, and thickness respectively, and where $T_{\text {depos }}$ is the deposition temperature. More than 10 thousands samples have been computed for each case, which ensures the convergence of the Monté-Carlo simulations. Indeed it can be seen in Fig. 14 that the distributions of both the resonance frequency and quality factor have converged after a few thousands resolutions.

The resulting statistical moments of the first resonance frequency and of the quality factor are reported in Table 3, and selected distributions of the first resonance frequency and of the quality factor are reported 


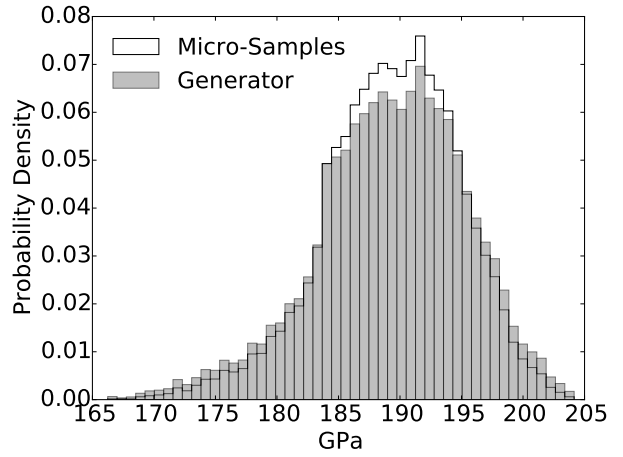

(a) $C_{\mathrm{M} 11}$

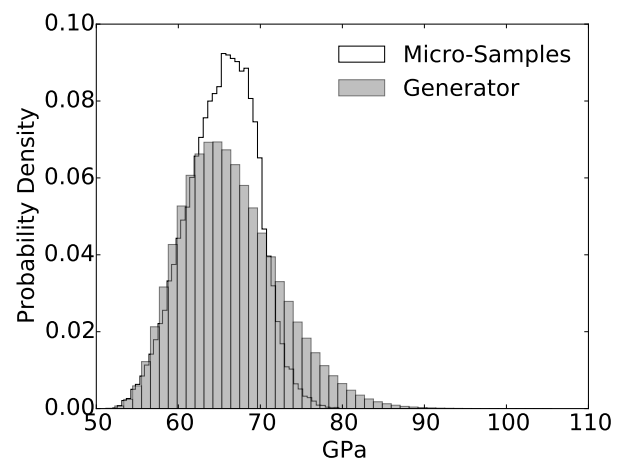

(c) $C_{\mathrm{M} 66}$

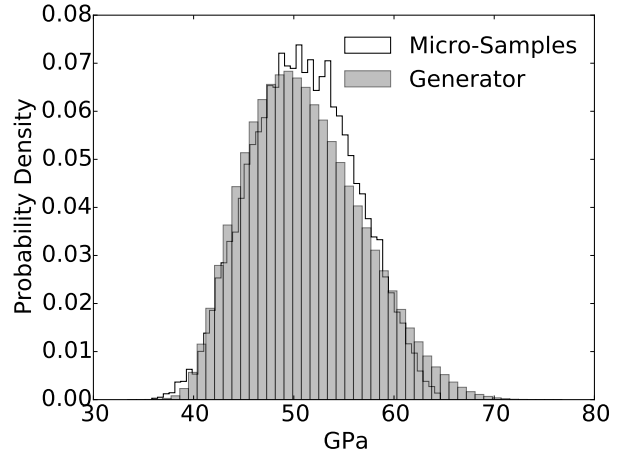

(b) $C_{\mathrm{M} 23}$

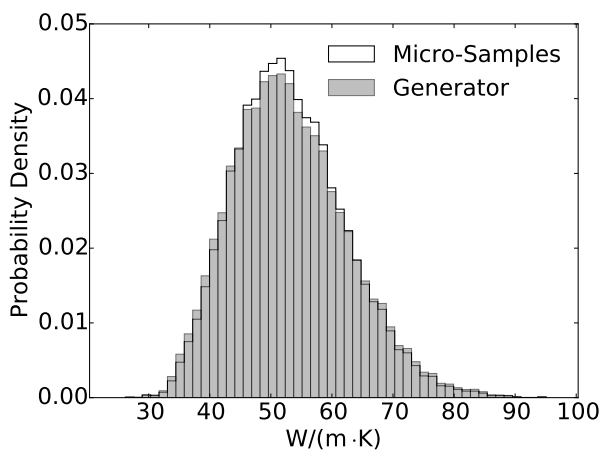

(d) $\kappa_{\mathrm{M} 33}$

Figure 9: Comparison of the histograms of $C_{\mathrm{M}_{i j}}$ and $\kappa_{\mathrm{M} l l}$ (no sum on $l$ ) between micro-samples and generated samples

Table 3: Statistical moments of the first resonance frequency $f_{1}$ and of the quality factor $Q$ for the different cases

\begin{tabular}{|c|c|c|c|c|c|c|}
\hline & $\mathbb{E}\left[f_{1}\right][\mathrm{MHz}]$ & $\boldsymbol{\sigma}_{f_{1}}[\mathrm{MHz}]$ & $\mathrm{COV}_{f_{1}}[\%]$ & $\mathbb{E}[Q]$ & $\boldsymbol{\sigma}_{Q}$ & $\mathrm{COV}_{Q}[\%]$ \\
\hline $\mathbf{1 5} \times \mathbf{3} \times \mathbf{2}, \mathbf{6 1 0}^{\circ} \mathbf{C}$ & 11.91 & 0.051 & 0.43 & 7771 & 82.95 & 1.067 \\
\hline $\mathbf{1 0} \times \mathbf{3} \times \mathbf{2}, \mathbf{6 1 0}{ }^{\circ} \mathbf{C}$ & 26.47 & 0.136 & 0.512 & 8634 & 93.35 & 1.081 \\
\hline $\mathbf{7 . 5} \times \mathbf{3} \times \mathbf{2}, \mathbf{6 1 0}^{\circ} \mathbf{C}$ & 46.20 & 0.254 & 0.551 & 10851 & 151.81 & 1.399 \\
\hline $\mathbf{1 5} \times \mathbf{3} \times \mathbf{1}, \mathbf{6 1 0}^{\circ} \mathbf{C}$ & 6.02 & 0.026 & 0.437 & 31855 & 481.9 & 1.513 \\
\hline $\mathbf{1 0} \times \mathbf{3} \times \mathbf{1}, \mathbf{6 1 0}^{\circ} \mathbf{C}$ & 13.53 & 0.071 & 0.523 & 15130 & 266.75 & 1.763 \\
\hline $\mathbf{5} \times \mathbf{3} \times \mathbf{1}, \mathbf{6 1 0}^{\circ} \mathbf{C}$ & 53.40 & 0.381 & 0.714 & 7145 & 116.20 & 1.626 \\
\hline $\mathbf{1 5} \times \mathbf{3} \times \mathbf{2}, \mathbf{6 3 0}^{\circ} \mathbf{C}$ & 11.89 & 0.068 & 0.575 & 9074 & 153.71 & 1.694 \\
\hline $\mathbf{1 5} \times \mathbf{3} \times \mathbf{2}, \mathbf{6 1 0}{ }^{\circ} \mathbf{C}, \mathbf{a n c h o r}$ & 10.63 & 0.037 & 0.349 & 7164 & 57.47 & 0.802 \\
\hline $\mathbf{1 0} \times \mathbf{3} \times \mathbf{2}, \mathbf{6 1 0}^{\circ} \mathbf{C}$, anchor & 22.38 & 0.088 & 0.393 & 7820 & 60.45 & 0.773 \\
\hline
\end{tabular}






(a) Without mapping correction

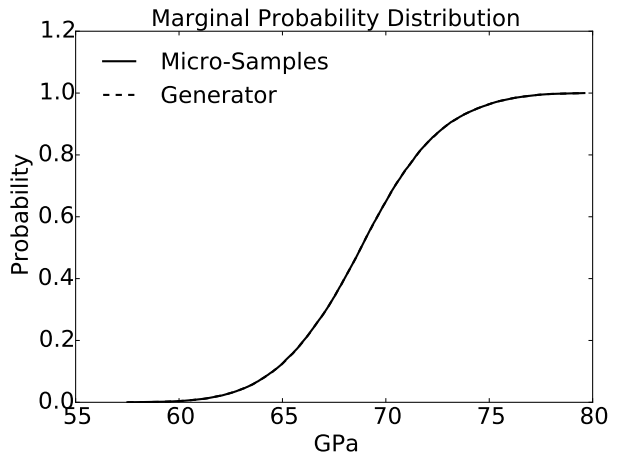

(b) With mapping correction

Figure 10: Comparison of marginal distributions of $C_{\mathrm{M} 55}$ between micro-samples and generated samples

in Fig. 15.

As predicted by other theories, the effect of the thickness on the quality factor is the most important geometrical one. When comparing the effect of the deposition temperature during the manufacturing process, the distribution of the eigen-frequency $f_{1}$ for a deposition temperature of $610^{\circ} \mathrm{C}$, Fig. 15 (a), is similar to the distribution of the eigen frequency $f_{1}$ for a deposition temperature of $630^{\circ} \mathrm{C}, \mathrm{Fig}$. $15(\mathrm{~g})$. However, its effect on the quality factor $Q$ is more sensitive, see Figs. 15(b) and $15(\mathrm{~h})$. This is mainly due to the effect of the grain size on the thermal conductivity.

\subsection{Cantilever with anchor}

To account for the effect of the boundary condition, we also consider a geometry with the anchor as displayed in Fig. 12(b). The boundary condition at the anchor clamp corresponds to Eq. (85).

We refer to the beam geometry and deposition temperature following the notation " $L \times w \times t, T_{\text {depos }}$, anchor", where $L, w$, and $t$ are the beam length, width, and thickness respectively, and where $T_{\text {depos }}$ is the deposition temperature. The anchor geometry is $4 \times 12 \times(2+t) \mu \mathrm{m}^{3}$. The MEMS is studied at room temperature. More than 2 thousands samples have been computed for this case.

The resulting statistical moments of the first resonance frequency and of the quality factor are reported in Table 3, and the distributions of the first resonance frequency and of the quality factor are reported in Fig. 16 for the $15 \times 3 \times 2,610^{\circ} \mathrm{C}$, anchor case.

As compared to the perfect clamping condition, Figs. 15(a) and 15(b), it can be seen that both the eigen frequency and quality factor means are lower when considering the anchor. Moreover, the coefficient of variation COV - the standard deviation normalized by the expectation- of the statistical distributions are lower with the anchor directly modeled, see Table 3. An illustration of the first vibration mode, with a temperature distribution is reported in Fig. 17. 


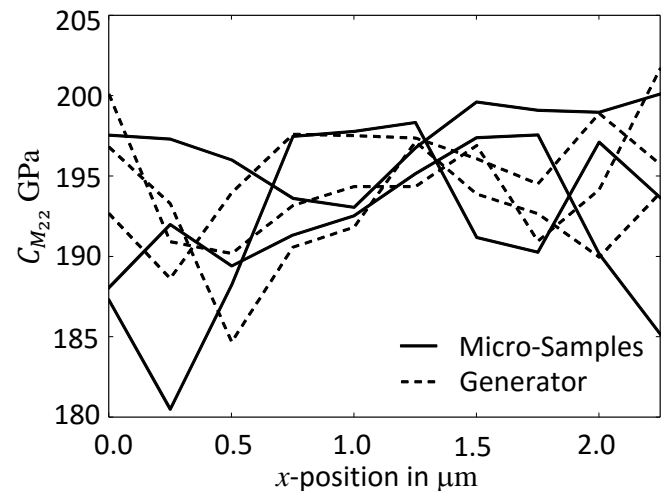

(a) Realizations of $C_{\mathrm{M} 22}$ at constant $y$-position

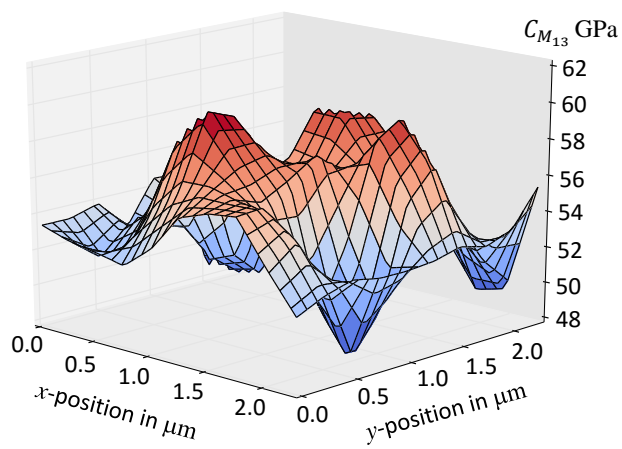

(c) Realizations of $C_{\mathrm{M} 13}$ from micro-samples

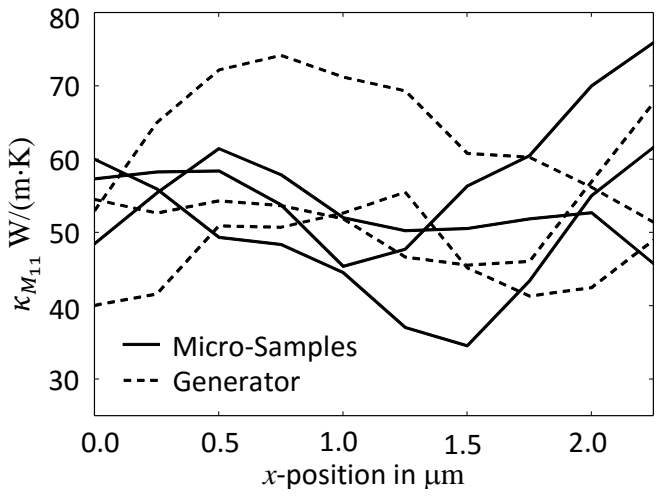

(b) Realizations of $\kappa_{\mathrm{M} 11}$ at constant $y$-position

Figure 11: Comparison of realizations of the random field between micro-samples and generated samples

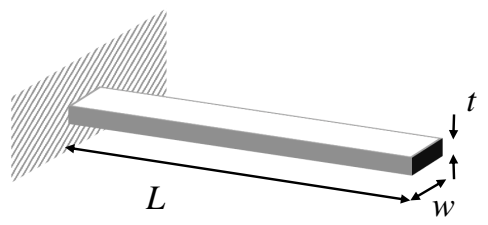

(a) Perfect clamp

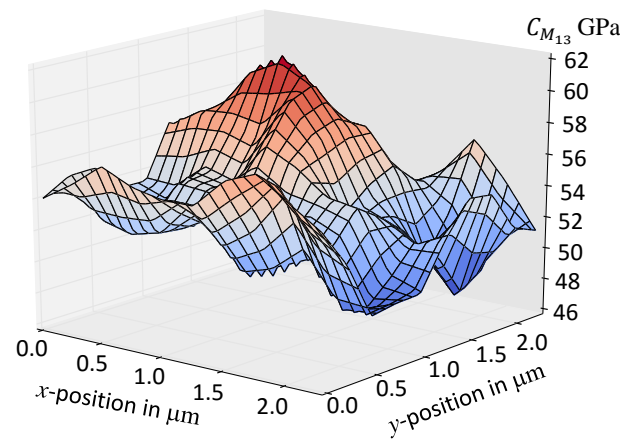

(d) Realizations of $C_{\mathrm{M} 13}$ from generator

\subsection{Comparison with analytical models}

In this section we compare the predictions of the stochastic multi-scale method to the deterministic Lifshitz and Roukes (LR) [21] thermo-elastic model. In this model, the length characterizing the conductivity 


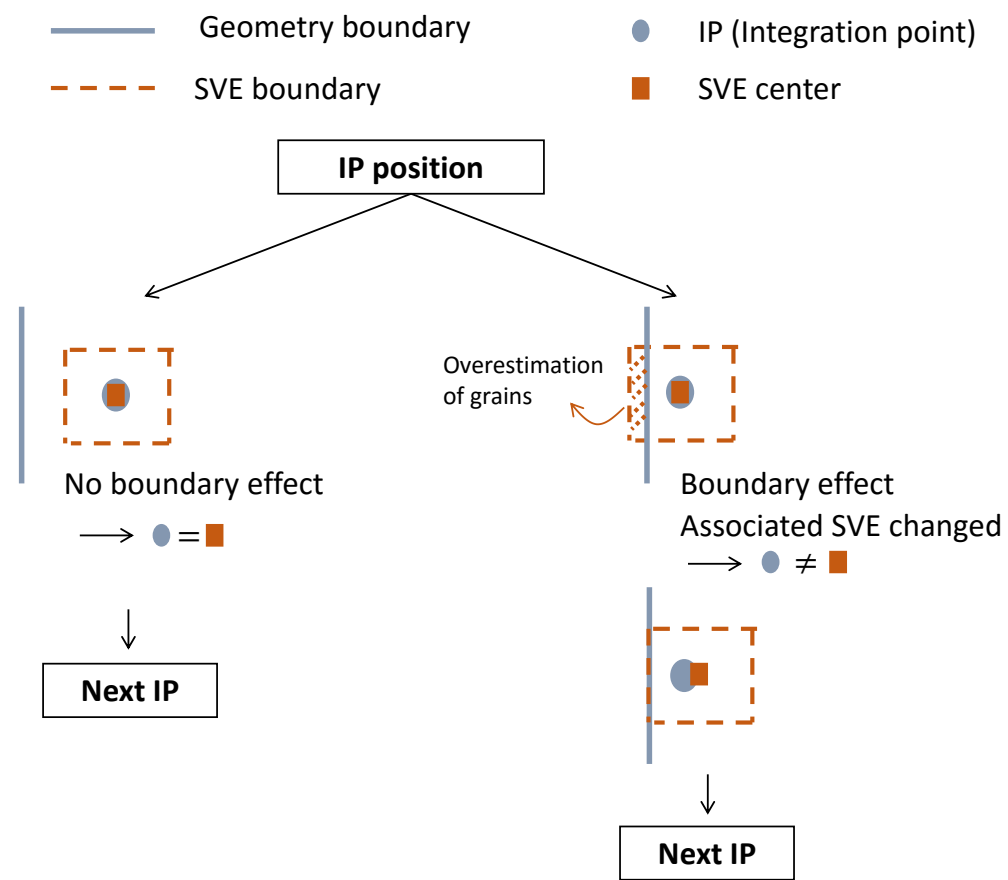

Figure 13: The boundary effect and its avoidance

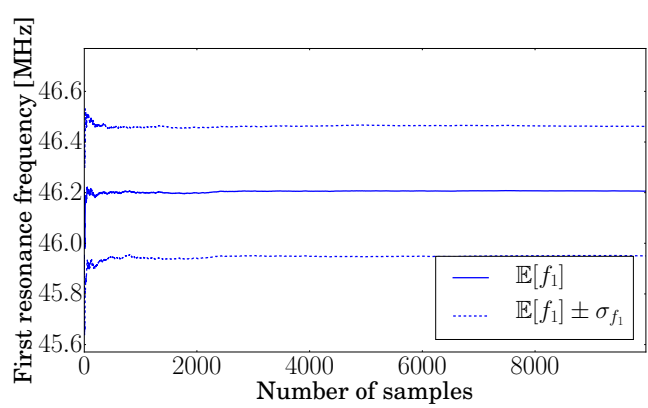

(a) Convergence of $f_{1}$

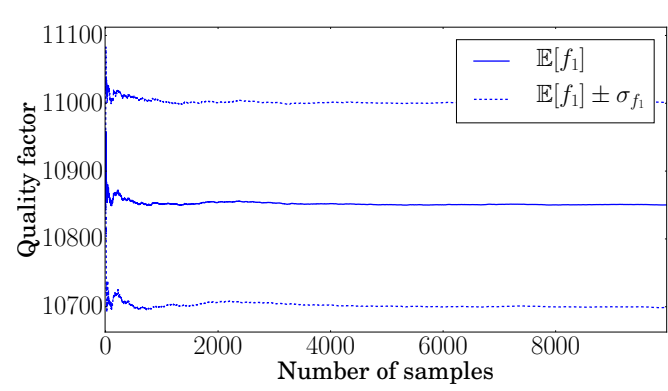

(b) Convergence of $Q$

Figure 14: Convergence of distribution statistical moments of (a) the resonance frequency and (b) of the quality factor of the perfectly clamped micro-resonator for the $7.5 \times 3 \times 2,610^{\circ} \mathrm{C}$ case

is assumed to be the beam thickness, and both the quality factor and the shift of the eigen-frequency of the resonator can be evaluated.

For a resonator, without considering damping, the resonant pulsations are equal to

$$
\varpi_{0, i}=\frac{k_{i}^{2}}{L^{2}} \sqrt{\frac{E t^{2}}{12 \rho}},
$$

where $k_{i}$ is the mode coefficient, $E$ is the Young's modulus along the beam length $L, t$ is the beam thickness, 


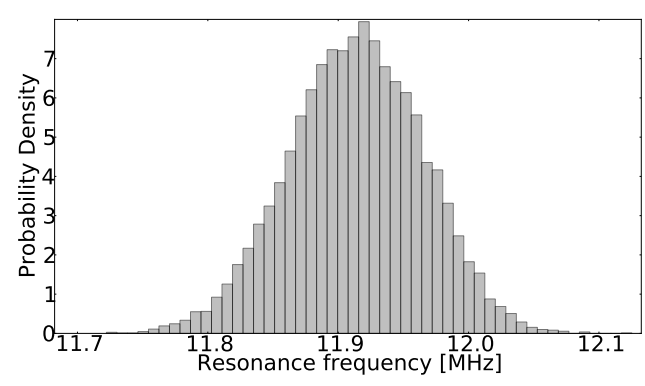

(a) $f_{1}$ for the $15 \times 3 \times 2,610^{\circ} \mathrm{C}$ case

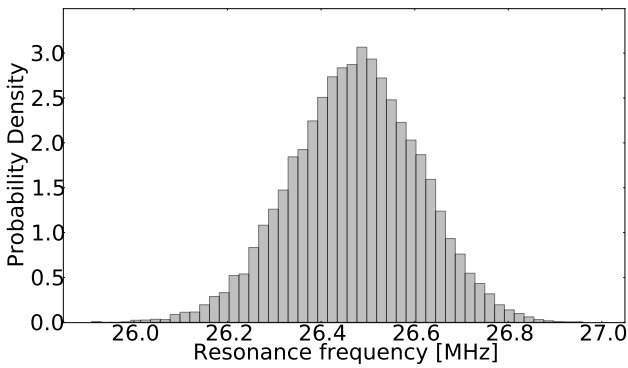

(c) $f_{1}$ for the $10 \times 3 \times 2,610^{\circ} \mathrm{C}$ case

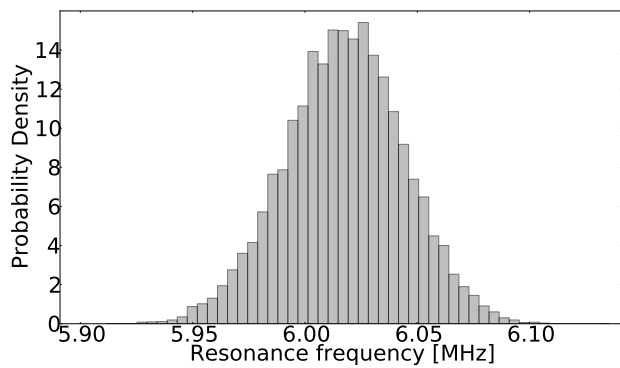

(e) $f_{1}$ for the $15 \times 3 \times 1,610^{\circ} \mathrm{C}$ case

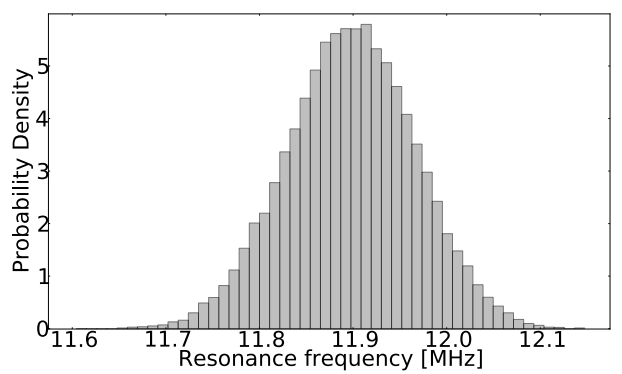

(g) $f_{1}$ for the $15 \times 3 \times 2,630^{\circ} \mathrm{C}$ case

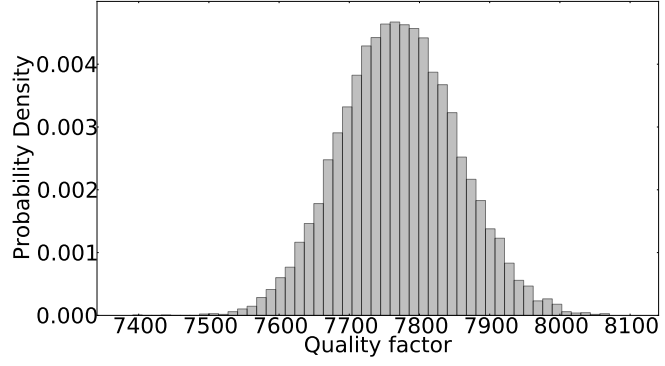

(b) $Q$ for the $15 \times 3 \times 2,610^{\circ} \mathrm{C}$ case

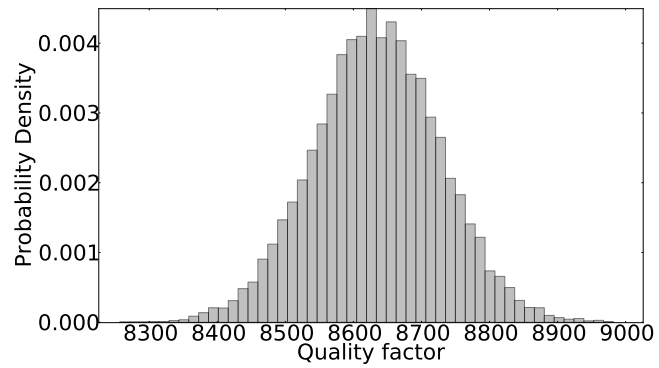

(d) $Q$ for the $10 \times 3 \times 2,610^{\circ} \mathrm{C}$ case

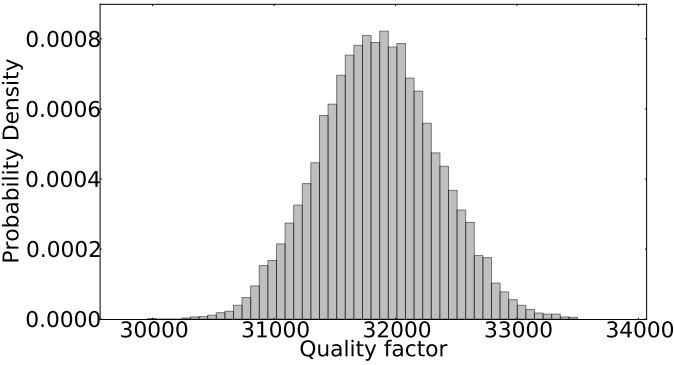

(f) $Q$ for the $15 \times 3 \times 1,610^{\circ} \mathrm{C}$ case

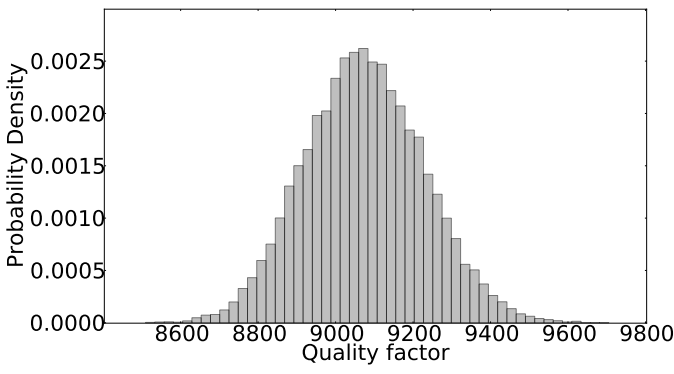

(h) $Q$ for the $15 \times 3 \times 2,630^{\circ} \mathrm{C}$ case

Figure 15: Histograms of the resonance frequency (left column) and of the quality factor (right column) of the perfectly clamped micro-resonator: (a) and (b) for the $15 \times 3 \times 2,610^{\circ} \mathrm{C}$ case, (c) and (d) $10 \times 3 \times 2,610^{\circ} \mathrm{C}$ case, (e) and (f) $5 \times 3 \times 1,610^{\circ} \mathrm{C}$ case, and $(\mathrm{g})$ and $(\mathrm{h}) 15 \times 3 \times 2,630^{\circ} \mathrm{C}$ case 


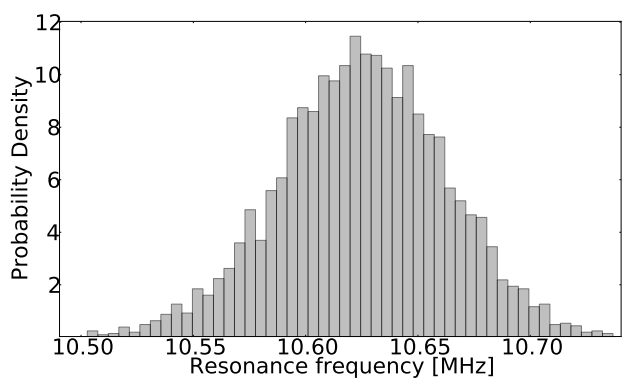

(a) $f_{1}$ for the $15 \times 3 \times 2,610^{\circ} \mathrm{C}$, anchor case

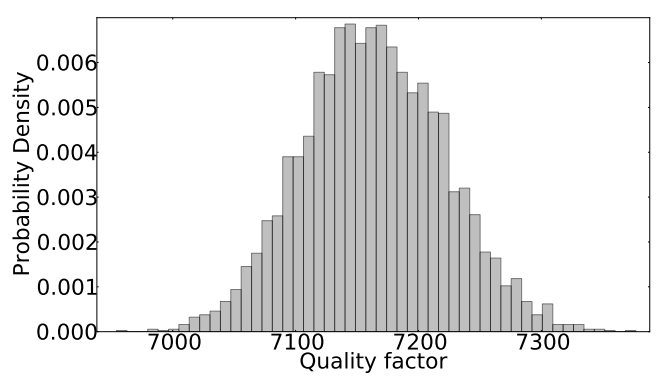

(b) $Q$ for the $15 \times 3 \times 2,610^{\circ} \mathrm{C}$, anchor case

Figure 16: Histograms of the (a) resonance frequency and of (b) the quality factor of the micro-resonator with the idealized anchor $\left(15 \times 3 \times 2,610^{\circ} \mathrm{C}\right.$, anchor case $)$

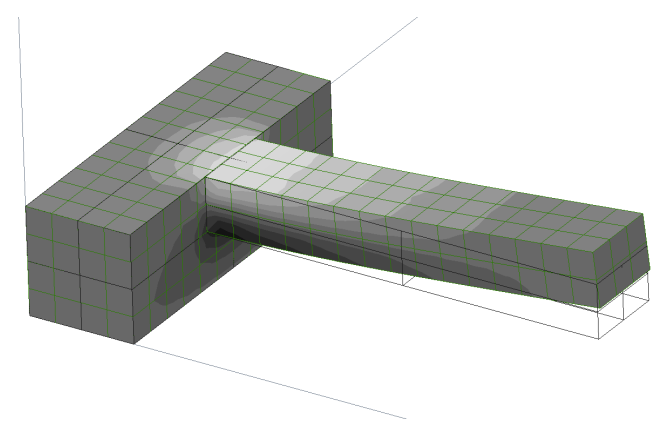

Figure 17: Illustration of the first vibrating mode with the temperature distribution (at a multiplicative constant) of the $15 \times 3 \times 2,610^{\circ} \mathrm{C}$, anchor case

the subscript 0 refers to the absence of damping, and where the subscript $i$ refers to the eigen mode number. For the first mode of a cantilever, we have $k_{1}=1.8751$.

Considering the conductivity across the thickness, let $\chi=\frac{\kappa}{\rho C_{v}}$ be the thermal diffusivity, with $\kappa$ the conductivity along the beam thickness, and let $\Delta_{E}=\frac{E \alpha^{2} T_{0}}{\rho C_{v}}$ be the relaxation strength, with $E$ and $\alpha$ respectively the Young's modulus and thermal expansion coefficient along the beam length.

Neglecting corrections of order $\Delta_{E}^{2}$, the thermo-elastic damping due to the conductivity across the thickness $t$ is thus characterized by the non-dimensional parameter

$$
\zeta=t \sqrt{\frac{\varpi_{0,1}}{2 \chi}} .
$$

According to LR model, the quality factor $Q$ and the shifted eigen pulsation $\varpi_{1}$ of the first mode are thus 
respectively given by

$$
\begin{aligned}
\frac{1}{Q} & =\Delta_{E}\left(\frac{6}{\zeta^{2}}-\frac{6}{\zeta^{3}} \frac{\sinh \zeta+\sin \zeta}{\cosh \zeta+\cos \zeta}\right), \text { and } \\
\varpi_{1} & =\varpi_{0,1}\left(1+\frac{\Delta_{E}}{2}\left(1-\frac{6}{\zeta^{3}} \frac{\sinh \zeta-\sin \zeta}{\cosh \zeta+\cos \zeta}\right)\right) .
\end{aligned}
$$

Besides the properties (84), we assume the following deterministic material properties for the LR model:

$$
E_{\mathrm{M}}=160 \mathrm{GPa}, \quad \kappa_{\mathrm{M}}=55 \mathrm{~W} /(\mathrm{m} \cdot \mathrm{K}),
$$

respectively the Young's modulus and the thermal conductivity.

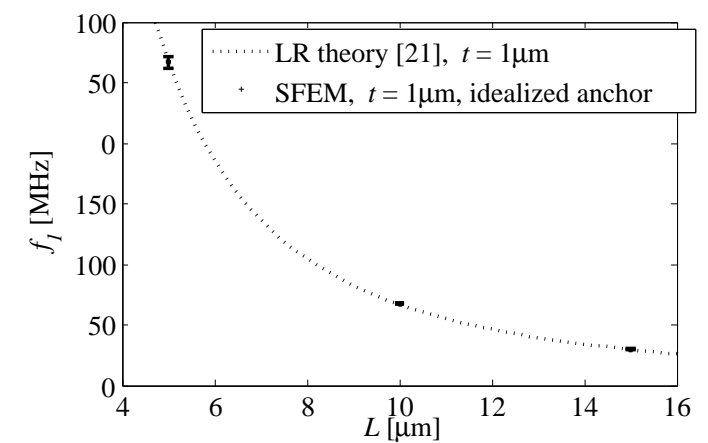

(a) $f_{1}$ for the $1 \mu$ m-thick beams



(c) $f_{1}$ for the $2 \mu$ m-thick beams

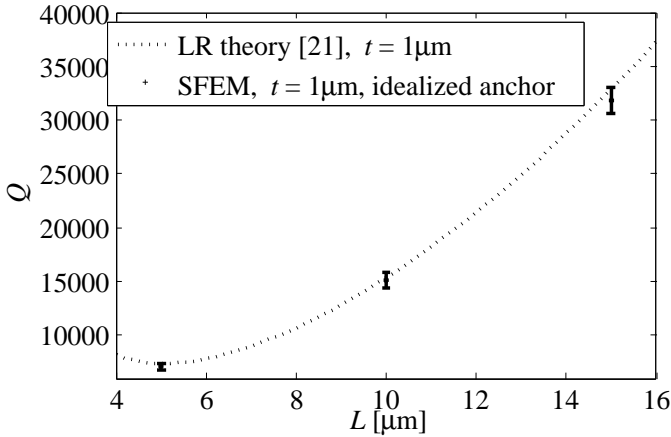

(b) $Q$ for the $1 \mu$ m-thick beams

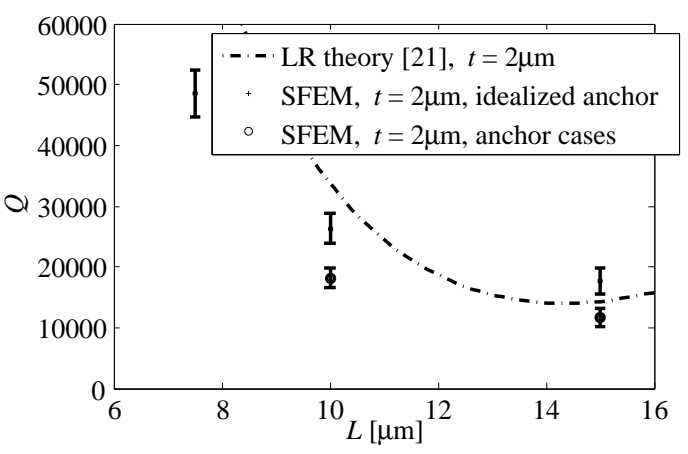

(d) $Q$ for the $2 \mu$ m-thick beams

Figure 18: Comparison between the LR model and the stochastic 3-scale predictions (99\% confidence intervals) for the microresonator (deposition temperature at $610^{\circ} \mathrm{C}$ ): resonance frequency (left column) and quality factor (right column) of the (a) and (b) $1 \mu \mathrm{m}$-thick resonators, and (c) and (d) $2 \mu \mathrm{m}$-thick resonators

The predictions of the LR model are compared to the stochastic 3-scale predictions in Fig. 18. On the one hand, for the $1 \mu \mathrm{m}$-thick resonators, see Figs. 18(a) and 18(b), the mean values of both the resonance frequency and of the quality factor follow the LR theory. Of course while the LR model is deterministic, the 3 -scale predictions exhibit an uncertainty. On the other hand, when considering the $2 \mu$ m-thick resonators, 
the mean values obtained with the stochastic 3-scale predictions do not follow the LR model anymore, see Figs. 18(c) and 18(d). Indeed, for the perfectly clamped conditions, if the mean resonance frequency is in good agreement with the LR theory, see Fig. 18(c), the mean quality factor is not in agreement with the LR model. This is due to the thermal conductivity along the beam length, which cannot be neglected anymore as what is done in the LR model. For the longer beam the boundary condition (85) induces an increase of the quality factor as we are getting close to isothermal conditions, while when the beam becomes shorter, the quality factor predicted with the 3D simulations becomes significantly lower than with the LR model because of the axial conductivity. Finally, when considering the effect of the clamping conditions, it can be seen that the simulations with the modelized anchor exhibit a lower resonence frequency due to the compliance of the boundary condition, but also a lower quality factor as the effect of the axial thermal conductivity becomes more important.

\section{Conclusions and perspectives}

A stochastic 3-scale thermo-mechanical approach was developped to propagate uncertainties from the micro-structure to the meso-scale through the characterization of a (meso-scale) random field, and then to a macro-scale quantity of interest by using as input of a SFEM the (meso-scale) random field. The phenomenon of thermo-elastic damping, which has an important effect on the dynamic behavior of micro-resonators, could thus be studied in a probabilistic way through the proposed three-scale process, by considering the uncertainties in the grain geometries and orientations of polycrystalline materials.

Toward this end, Stochastic Volume Elements were first generated under the form of columnar Voronoï tessellations, with a grain size and orientation distributions following experimental measurements obtained on poly-silicon layers deposited at two different temperatures.

A stochastic coupled thermo-mechanical homogenization was conducted to extract meso-scale thermomechanical properties realizations. A stochastic model was then built upon those realizations by combining a spectral generator with a non-Gaussian mapping. As a result, (spatially) correlated meso-scale random fields of the thermo-mechanical properties can be generated in an efficient computational way.

From the generated meso-scale random fields, thermo-mechanical stochastic finite elements were then used to study the probabilistic behavior of MEMS resonators. In particular the thermo-mechanical damping effect on the quality factor of micro-resonators was analyzed and compared to analytical models. Besides accounting for the uncertainties, the 3D model allows to account for the axial conductivity, contrarily to the analytical models.

Because of the efficiency of the method, it was possible to study in a probabilistic way several resonator geometries, the effect of the boundary conditions, and of the deposition temperatures, on the device vibration behavior. 


\section{Appendix A. Thermo-mechanical formulation}

In the thermo-mechanics context, besides the linear momentum Eq. (1), the deformable solid behavior is governed by the (localized) energy balance per unit volume [28]

$$
\rho \dot{e}-\rho s-\boldsymbol{\sigma}: \dot{\varepsilon}+\nabla \cdot \boldsymbol{q}=0
$$

where $e$ is the internal energy per unit mass, $\boldsymbol{\sigma}$ is the Cauchy stress tensor, $\varepsilon$ is the Cauchy strain tensor, $s$ is the heat source per unit mass, and $\boldsymbol{q}$ is the thermal flux vector.

The (localized) Clausius-Duhem inequality reads

$$
\rho \dot{\eta}+\nabla \cdot\left(\frac{\boldsymbol{q}}{T}\right)-\frac{\rho s}{T} \geq 0,
$$

where $\eta$ is the entropy per unit mass and $T$ is the absolute temperature. This last relation can be rewritten as

$$
\rho \dot{\eta}-\frac{1}{T}(\rho s-\nabla \cdot \boldsymbol{q})-\frac{1}{T^{2}} \boldsymbol{q} \cdot \nabla T \geq 0 .
$$

Multiplying this last equation by $T$ and subtracting it from (A.1) yields

$$
\rho(\dot{e}-T \dot{\eta})-\boldsymbol{\sigma}: \dot{\varepsilon}+\frac{1}{T}(\boldsymbol{q} \cdot \nabla T) \leq 0 .
$$

Defining the specific free energy $\phi=e-T \eta$ allows writing

$$
\dot{\phi}=\dot{e}-T \dot{\eta}-\dot{T} \eta,
$$

and Eq. (A.4) allows defining the dissipation per unit volume $\mathcal{D}$ as

$$
-\mathcal{D}=\rho(\dot{\phi}+\dot{T} \eta)-\boldsymbol{\sigma}: \dot{\varepsilon}+\frac{1}{T}(\boldsymbol{q} \cdot \nabla T) \leq 0 .
$$

The thermodynamics forces are set to be

$$
\left(\frac{\partial \phi}{\partial T}\right)_{\varepsilon}=-\eta \text { and } \rho\left(\frac{\partial \phi}{\partial \varepsilon}\right)_{T}=\boldsymbol{\sigma}
$$

and are completed by the condition $\boldsymbol{q} \cdot \nabla T \leq 0$. By differentiation of the specific free energy one thus has

$$
\rho \dot{\phi}=\sigma: \dot{\varepsilon}-\rho \eta \dot{T},
$$

which, combined to Eq. (A.5), leads to the so-called Gibbs relation

$$
\rho \dot{e}=\sigma: \dot{\varepsilon}+\rho T \dot{\eta} .
$$

Combining this relation to Eq. (A.1) leads to the expression of the entropy rate

$$
\rho T \dot{\eta}=\rho \dot{e}-\boldsymbol{\sigma}: \dot{\varepsilon}=\rho s-\nabla \cdot \boldsymbol{q} .
$$


Eventually, using Eqs. (A.5), the entropy relation (A.10), and the energy balance (A.1) in the dissipation expression (A.6) yields

$$
\mathcal{D}=-\rho \dot{e}+\rho s-\nabla \cdot \boldsymbol{q}+\boldsymbol{\sigma}: \dot{\boldsymbol{\varepsilon}}-\frac{1}{T}(\boldsymbol{q} \cdot \nabla T)=-\frac{1}{T}(\boldsymbol{q} \cdot \nabla T)
$$

Let us now consider thermo-elasticity with $\mathcal{S}=\rho \eta$ the entropy per unit volume, $\rho e=\rho C_{v} T$ with $C_{v}$ the heat capacity per unit mass, and $\mathcal{F}(T, \varepsilon)=\rho \phi=\rho C_{v} T-T \mathcal{S}$ the Helmholtz free energy defined in Eq. (4) for constant material parameters. The framework reported here above thus reduces to the set of equations reported in Section 2.1.1.

\section{Appendix B. The definition of sub-matrices in finite element formula}

The sub-matrices in Eq. (23) are defined by

$$
\begin{aligned}
\boldsymbol{M} & =\int_{\Omega} \rho \boldsymbol{N}_{u}^{\mathrm{T}} \boldsymbol{N}_{u} \mathrm{~d} \Omega, \\
\boldsymbol{D}_{\vartheta u} & =T_{0} \int_{\Omega} \boldsymbol{N}_{\vartheta}^{\mathrm{T}} \boldsymbol{\alpha} \boldsymbol{C} \boldsymbol{B}_{u} \mathrm{~d} \Omega, \\
\boldsymbol{D}_{\vartheta \vartheta} & =\int_{\Omega} \rho C_{v} \boldsymbol{N}_{\vartheta}^{\mathrm{T}} \boldsymbol{N}_{\vartheta} \mathrm{d} \Omega, \\
\boldsymbol{K}_{u u} & =\int_{\Omega} \boldsymbol{B}_{u}^{\mathrm{T}} \boldsymbol{C} \boldsymbol{B}_{u} \mathrm{~d} \Omega, \\
\boldsymbol{K}_{u \vartheta} & =-\int_{\Omega} \boldsymbol{B}_{u}^{\mathrm{T}} \boldsymbol{C} \boldsymbol{\alpha} \boldsymbol{N}_{\vartheta} \mathrm{d} \Omega, \text { and } \\
\boldsymbol{K}_{\vartheta \vartheta} & =\int_{\Omega} \boldsymbol{B}_{\vartheta}^{\mathrm{T}} \boldsymbol{\kappa} \boldsymbol{B}_{\vartheta} \mathrm{d} \Omega .
\end{aligned}
$$

The external force vectors are easily obtained from

$$
\begin{aligned}
& \boldsymbol{F}_{u}=\int_{\Omega} \boldsymbol{N}_{u}^{\mathrm{T}} \rho \boldsymbol{b} \mathrm{d} \Omega+\int_{\Gamma_{\mathcal{T}}} \boldsymbol{N}_{u}^{\mathrm{T}} \boldsymbol{\mathcal { T }} \mathrm{d} \Gamma, \\
& \boldsymbol{F}_{\vartheta}=-\int_{\Gamma_{q}^{T}} \boldsymbol{N}_{\vartheta}^{\mathrm{T}} \mathcal{Q} \mathrm{d} \Gamma^{T} .
\end{aligned}
$$

\section{Appendix C. The constrained micro-scale finite element formulation}

\section{Appendix C.1. Definition of the constraints}

The specified kinematic admissible boundary conditions applied on the finite element discretization (41) of the SVE in this work are the Periodic Boundary Conditions (PBCs) (58) of displacement $\boldsymbol{u}_{\mathrm{m}}$ and temperature $\vartheta_{\mathrm{m}}$, completed by the consistency condition (51).

The degrees of freedom of the micro-scale problem (we drop the subscript ' $m$ ' for conciseness) are organized in terms of $\boldsymbol{u}_{c}, \boldsymbol{u}_{b}$, and $\boldsymbol{u}_{i}$, respectively the corner nodal displacements, the boundary nodal 
displacements, and the internal nodal displacements, and in terms of $\boldsymbol{\vartheta}_{c}, \boldsymbol{\vartheta}_{b}$, and $\boldsymbol{\vartheta}_{i}$, respectively the corner nodal temperatures, the boundary nodal temperatures, and the internal nodal temperatures.

The kinematics constraints are defined by $\mathbb{F}_{\mathrm{M}}=\boldsymbol{u}_{\mathrm{M}} \otimes \nabla_{\mathrm{M}}$ the macroscopic displacement gradient, with $\boldsymbol{F}_{\mathrm{M}}$ the nine components of $\mathbb{F}_{\mathrm{M}}$ written under a vectorial form, by $\nabla_{\mathrm{M}} \vartheta_{\mathrm{M}}$ the macroscopic temperature gradient, and by $\vartheta_{\mathrm{M}}$ the macroscopic temperature.

The periodic boundary conditions (58) are first used to define the constraints on the displacement field at the corner nodes $p$, leading to

$$
\boldsymbol{u}_{c}^{p}=\left[\begin{array}{ccc}
\boldsymbol{x}^{p \mathrm{~T}} & \mathbf{0}_{1 \times 3} & \mathbf{0}_{1 \times 3} \\
\mathbf{0}_{1 \times 3} & \boldsymbol{x}^{p \mathrm{~T}} & \mathbf{0}_{1 \times 3} \\
\mathbf{0}_{1 \times 3} & \mathbf{0}_{1 \times 3} & \boldsymbol{x}^{p \mathrm{~T}}
\end{array}\right] \boldsymbol{F}_{\mathrm{M}}=\boldsymbol{S}_{u_{c}}^{p} \boldsymbol{F}_{\mathrm{M}}
$$

or after assembly

$$
\boldsymbol{u}_{c}-\boldsymbol{S}_{u_{c}} \boldsymbol{F}_{\mathrm{M}}=0 .
$$

The periodic boundary conditions (58) are then used to define the constraints on the displacement field on two opposite boundary nodes $p^{+}$and $p^{-}$, leading to

$$
\boldsymbol{u}_{b}^{p^{+}}-\boldsymbol{u}_{b}^{p^{-}}=\left[\begin{array}{ccc}
\boldsymbol{x}^{p^{+\mathrm{T}}}-\boldsymbol{x}^{p^{-\mathrm{T}}} & \mathbf{0}_{1 \times 3} & \mathbf{0}_{1 \times 3} \\
\mathbf{0}_{1 \times 3} & \boldsymbol{x}^{p^{\mathrm{T}^{\mathrm{T}}}}-\boldsymbol{x}^{p^{-\mathrm{T}}} & \mathbf{0}_{1 \times 3} \\
\mathbf{0}_{1 \times 3} & \mathbf{0}_{1 \times 3} & \boldsymbol{x}^{p^{\mathrm{T}^{\mathrm{T}}}}-\boldsymbol{x}^{p^{-\mathrm{T}}}
\end{array}\right] \boldsymbol{F}_{\mathrm{M}}=\boldsymbol{S}_{u_{b}}^{p} \boldsymbol{F}_{\mathrm{M}},
$$

or after assembly

$$
\boldsymbol{C}_{u_{b}} \boldsymbol{u}_{b}-\boldsymbol{S}_{u_{b}} \boldsymbol{F}_{\mathrm{M}}=0 .
$$

In case of a non-periodic mesh, the use of the interpolation method developed in [31] yields a similar relation which involves the corner nodes as well

$$
\boldsymbol{C}_{u_{c}} \boldsymbol{u}_{c}+\boldsymbol{C}_{u_{b}} \boldsymbol{u}_{b}-\boldsymbol{S}_{u_{b}} \boldsymbol{F}_{\mathrm{M}}=0 .
$$

Concerning the temperature field, the rigid mode is constrained by the condition (51), and not by a constraint similar to (C.2). The thermal part of the periodic boundary conditions (58) thus involves both corner and boundary nodal temperatures, which results in

$$
\boldsymbol{\vartheta}_{b(c)}^{p^{+}}-\boldsymbol{\vartheta}_{b(c)}^{p^{-}}=\left[\boldsymbol{x}^{p^{+\mathrm{T}}}-\boldsymbol{x}^{p^{-\mathrm{T}}}\right] \nabla \vartheta_{\mathrm{M}}=\boldsymbol{S}_{\vartheta_{b(c)}^{p}}^{p} \nabla \vartheta_{\mathrm{M}},
$$

or after assembly

$$
\boldsymbol{C}_{\vartheta_{c}} \boldsymbol{\vartheta}_{c}+\boldsymbol{C}_{\vartheta_{b}} \boldsymbol{\vartheta}_{b}-\boldsymbol{S}_{\vartheta_{b}} \nabla \vartheta_{\mathrm{M}}=0
$$

which remains valid in case of a non-periodic mesh when using the interpolation method. Finally, the last condition (51) is discretized by considering the corner, boundary, and internal nodal temperatures as

$$
\frac{1}{V(\omega)} \int_{\omega} \rho_{\mathrm{m}} C_{v \mathrm{~m}} \boldsymbol{N}_{\vartheta}^{p} \mathrm{~d} V \boldsymbol{\vartheta}_{(b c i)}^{p}=\boldsymbol{C}_{\Theta_{(b c i)}}^{p} \boldsymbol{\vartheta}_{(b c i)}^{p}=\rho_{\mathrm{M}} C_{v \mathrm{M}} \vartheta_{\mathrm{M}}=S_{\Theta_{b}} \vartheta_{\mathrm{M}},
$$


or again

$$
\boldsymbol{C}_{\Theta_{c}} \boldsymbol{\vartheta}_{c}+\boldsymbol{C}_{\Theta_{b}} \boldsymbol{\vartheta}_{b}+\boldsymbol{C}_{\Theta_{i}} \boldsymbol{\vartheta}_{i}-S_{\Theta_{b}} \vartheta_{\mathrm{M}}=0
$$

As the constraints (C.5), (C.7), and (C.9), have the same structure, the related nodal fields are groups together into the vector $\boldsymbol{\varphi}_{b}^{\mathrm{T}}=\left[\boldsymbol{u}_{b}^{\mathrm{T}} \boldsymbol{\vartheta}_{c}^{\mathrm{T}} \boldsymbol{\vartheta}_{b}^{\mathrm{T}} \boldsymbol{\vartheta}_{i}^{\mathrm{T}}\right]$, and the micro-structural problem (41) is thus organized in terms of the nodal unknowns

$$
\boldsymbol{\varphi}=\left[\begin{array}{lll}
\boldsymbol{u}_{c}^{\mathrm{T}} & \boldsymbol{\varphi}_{b}^{\mathrm{T}} & \boldsymbol{u}_{i}^{\mathrm{T}}
\end{array}\right]^{\mathrm{T}}
$$

which are the constrained, dependent, and independent unknowns respectively, of the nodal forces

$$
\boldsymbol{F}=\left[\begin{array}{lll}
\boldsymbol{F}_{u_{c}}^{\mathrm{T}} & \boldsymbol{F}_{\varphi_{b}}^{\mathrm{T}} & \boldsymbol{F}_{u_{i}}^{\mathrm{T}}
\end{array}\right]^{\mathrm{T}}
$$

and of the stiffness matrix

$$
\boldsymbol{K}=\left[\begin{array}{ccc}
\boldsymbol{K}_{u_{c} u_{c}} & \boldsymbol{K}_{u_{c} \varphi_{b}} & \boldsymbol{K}_{u_{c} u_{i}} \\
\boldsymbol{K}_{\varphi_{b} u_{c}} & \boldsymbol{K}_{\varphi_{b} \varphi_{b}} & \boldsymbol{K}_{\varphi_{b} u_{i}} \\
\boldsymbol{K}_{u_{i} u_{c}} & \boldsymbol{K}_{u_{i} \varphi_{b}} & \boldsymbol{K}_{u_{i} u_{i}}
\end{array}\right]
$$

The constraints (C.2), (C.5), (C.7), and (C.9) are reorganized, leading to

$$
\begin{aligned}
0 & =\boldsymbol{u}_{c}-\boldsymbol{S}_{u_{c}} \boldsymbol{F}_{\mathrm{M}}=\boldsymbol{u}_{c}-\boldsymbol{S}_{\varphi_{c}} \boldsymbol{K}_{\mathrm{M}}, \\
0 & =\boldsymbol{C}_{\varphi_{c}} \boldsymbol{u}_{c}+\boldsymbol{C}_{\varphi_{b}} \boldsymbol{\varphi}_{b}-\boldsymbol{S}_{\varphi_{b}} \boldsymbol{K}_{\mathrm{M}},
\end{aligned}
$$

where

$$
\begin{aligned}
& \boldsymbol{S}_{\varphi_{b}}=\left[\begin{array}{ccc}
\boldsymbol{S}_{u_{b}} & \mathbf{0}_{\frac{N_{u_{b}}}{2} \times 3} & \mathbf{0}_{\frac{N_{u_{b}}}{2} \times 1} \\
\frac{\mathbf{0}_{\vartheta_{\vartheta_{c}}+N_{\vartheta_{b}}} \times 9}{2} & \boldsymbol{S}_{\vartheta_{b}} & \mathbf{0}_{\frac{N_{\vartheta_{c}}+N_{\vartheta_{b}}}{2} \times 1} \\
\mathbf{0}_{1 \times 9} & \mathbf{0}_{1 \times 3} & S_{\Theta_{b}}
\end{array}\right], \boldsymbol{S}_{\varphi_{c}}^{\mathrm{T}}=\left[\begin{array}{c}
\boldsymbol{S}_{u_{c}}^{\mathrm{T}} \\
\boldsymbol{0}_{3 \times N_{u_{c}}} \\
\mathbf{0}_{1 \times N_{u_{c}}}
\end{array}\right], \\
& \boldsymbol{C}_{\varphi_{b}}=\left[\begin{array}{cccc}
\boldsymbol{C}_{u_{b}} & \mathbf{0}_{\frac{N u_{b}}{2} \times N_{\vartheta_{c}}} & \mathbf{0}_{\frac{N_{u_{b}}}{2} \times N_{\vartheta_{b}}} & \mathbf{0}_{\frac{N u_{b}}{2} \times N_{\vartheta_{i}}} \\
\mathbf{0}_{\frac{N_{u_{c}}+N_{u_{b}}}{2} \times N_{u_{b}}} & \boldsymbol{C}_{\vartheta_{c}} & \boldsymbol{C}_{\vartheta_{b}} & \mathbf{0}_{\frac{N_{u_{c}}+N_{u_{b}}}{2} \times N_{\vartheta_{i}}} \\
\boldsymbol{0}_{1 \times N_{u_{b}}} & \boldsymbol{C}_{\Theta_{c}} & \boldsymbol{C}_{\Theta_{b}} & \boldsymbol{C}_{\Theta_{i}}
\end{array}\right], \\
& \boldsymbol{C}_{\varphi_{c}}=\left[\begin{array}{c}
\boldsymbol{C}_{u_{c}} \\
\boldsymbol{0}_{\frac{N_{u_{c}}+N_{u_{b}}}{2} \times N_{u_{c}}} \\
\mathbf{0}_{1 \times N_{u_{c}}}
\end{array}\right], \boldsymbol{K}_{\mathrm{M}}=\left[\begin{array}{c}
\boldsymbol{F}_{\mathrm{M}} \\
\nabla_{\mathrm{M}} \vartheta_{M} \\
\vartheta_{\mathrm{M}}
\end{array}\right],
\end{aligned}
$$

with $N_{u_{c}}$.. referring to the number of constraints per type (constrained or dependent degrees of freedom). 


\section{Appendix C.2. Resolution of the constrained micro-scale finite element problem}

The resolution of the constrained micro-scale BVP follows the multiple-constraint projection method described in [1] and the condensation method developed in [30]. The functional related to the constrained micro-scale problem is defined, using the conditions (C.13-C.14), by

$$
\Psi=\frac{1}{2} \boldsymbol{\varphi}^{\mathrm{T}} \boldsymbol{K} \boldsymbol{\varphi}-\left[\boldsymbol{u}_{c}-\boldsymbol{S}_{\varphi_{c}} \boldsymbol{K}_{\mathrm{M}}\right]^{\mathrm{T}} \boldsymbol{\lambda}_{u_{c}}-\left[\boldsymbol{C}_{\varphi_{c}} \boldsymbol{u}_{c}+\boldsymbol{C}_{\varphi_{b}} \boldsymbol{\varphi}_{b}-\boldsymbol{S}_{\varphi_{b}} \boldsymbol{K}_{\mathrm{M}}\right]^{\mathrm{T}} \boldsymbol{\lambda}_{\varphi_{b}},
$$

with the Lagrange multipliers $\boldsymbol{\lambda}_{u_{c}}$ and $\boldsymbol{\lambda}_{\varphi_{b}}$ which are respectively related to the corner displacement constraints and to the dependent unknowns constraints.

First, the minimization of (C.18) with respect to the nodal unknowns yields

$$
\begin{aligned}
0 & =\frac{\partial \Psi}{\partial \boldsymbol{u}_{i}}=\boldsymbol{F}_{u_{i}}, \\
0 & =\frac{\partial \Psi}{\partial \boldsymbol{\varphi}_{b}}=\boldsymbol{F}_{\varphi_{b}}-\boldsymbol{C}_{\varphi_{b}}^{\mathrm{T}} \boldsymbol{\lambda}_{\varphi_{b}}, \\
0 & =\frac{\partial \Psi}{\partial \boldsymbol{u}_{c}}=\boldsymbol{F}_{u_{c}}-\boldsymbol{\lambda}_{u_{c}}-\boldsymbol{C}_{\varphi_{c}}^{\mathrm{T}} \boldsymbol{\lambda}_{\varphi_{b}} .
\end{aligned}
$$

Defining $\boldsymbol{R}_{\varphi_{b}}^{\mathrm{T}}=\left(\boldsymbol{C}_{\varphi_{b}} \boldsymbol{C}_{\varphi_{b}}^{\mathrm{T}}\right)^{-1} \boldsymbol{C}_{\varphi_{b}}$, the solution of the system (C.19-C.21) reads

$$
\begin{aligned}
\boldsymbol{\lambda}_{\varphi_{b}} & =\boldsymbol{R}_{\varphi_{b}}^{\mathrm{T}} \boldsymbol{F}_{\varphi_{b}}, \\
\boldsymbol{\lambda}_{u_{c}} & =\boldsymbol{F}_{u_{c}}-\boldsymbol{C}_{\varphi_{c}}^{\mathrm{T}} \boldsymbol{R}_{\varphi_{b}}^{\mathrm{T}} \boldsymbol{F}_{\varphi_{b}} .
\end{aligned}
$$

\section{Appendix C.3. Homogenized stress and flux}

The homogenized stress tensor (45) is evaluated in the matrix form as

$$
\boldsymbol{\Sigma}_{\mathrm{M}}=\frac{1}{V(\omega)}\left(\frac{\partial \Psi}{\partial \boldsymbol{F}_{\mathrm{M}}}\right)=\frac{1}{V(\omega)}\left[\begin{array}{lll}
\boldsymbol{I}_{9 \times 9} & \mathbf{0}_{9 \times 3} & \mathbf{0}_{0 \times 1}
\end{array}\right]\left\{\boldsymbol{S}_{\varphi_{c}}^{\mathrm{T}} \boldsymbol{\lambda}_{u_{c}}+\boldsymbol{S}_{\varphi_{b}}^{\mathrm{T}} \boldsymbol{\lambda}_{\varphi_{b}}\right\}
$$

Similarly, the homogenized thermal flux (47) is evaluated as

$$
\boldsymbol{q}_{\mathrm{M}}=\frac{1}{V(\omega)}\left(\frac{\partial \Psi}{\partial \nabla_{\mathrm{M}} \vartheta_{\mathrm{M}}}\right)=\frac{1}{V(\omega)}\left[\begin{array}{ccc}
\mathbf{0}_{3 \times 9} & \boldsymbol{I}_{3 \times 3} & \mathbf{0}_{3 \times 1}
\end{array}\right]\left\{\boldsymbol{S}_{\varphi_{c}}^{\mathrm{T}} \boldsymbol{\lambda}_{u_{c}}+\boldsymbol{S}_{\varphi_{b}}^{\mathrm{T}} \boldsymbol{\lambda}_{\varphi_{b}}\right\} .
$$

\section{Appendix C.4. Homogenized material properties}

To compute the material operators, the stationary point of (C.18) is linearized with respect to the kinematics constraints $\boldsymbol{K}_{\mathrm{M}}$. Linearizing Eqs. (C.19-C.21) respectively yields

$$
\begin{aligned}
\boldsymbol{K}_{u_{i} u_{i}} \delta \boldsymbol{u}_{i}+\boldsymbol{K}_{u_{i} u_{c}} \delta \boldsymbol{u}_{c}+\boldsymbol{K}_{u_{i} \varphi_{b}} \delta \boldsymbol{\varphi}_{b} & =0 \\
\boldsymbol{K}_{\varphi_{b} u_{i}} \delta \boldsymbol{u}_{i}+\boldsymbol{K}_{\varphi_{b} u_{c}} \delta \boldsymbol{u}_{c}+\boldsymbol{K}_{\varphi_{b} \varphi_{b}} \delta \boldsymbol{\varphi}_{b} & =\boldsymbol{C}_{\varphi_{b}}^{\mathrm{T}} \delta \boldsymbol{\lambda}_{\varphi_{b}}, \\
\boldsymbol{K}_{u_{c} u_{i}} \delta \boldsymbol{u}_{i}+\boldsymbol{K}_{u_{c} u_{c}} \delta \boldsymbol{u}_{c}+\boldsymbol{K}_{u_{c} \varphi_{b}} \delta \boldsymbol{\varphi}_{b} & =\delta \boldsymbol{\lambda}_{u_{c}}+\boldsymbol{C}_{\varphi_{c}}^{\mathrm{T}} \delta \boldsymbol{\lambda}_{\varphi_{b}} .
\end{aligned}
$$

As Eq. (C.26) implies

$$
\delta \boldsymbol{u}_{i}=-\boldsymbol{K}_{u_{i} u_{i}}^{-1} \boldsymbol{K}_{u_{i} u_{c}} \delta \boldsymbol{u}_{c}-\boldsymbol{K}_{u_{i} u_{i}}^{-1} \boldsymbol{K}_{u_{i} \varphi_{b}} \delta \varphi_{b},
$$


the two remaining equations (C.27-C.28) are rewritten as

$$
\begin{gathered}
\left(\boldsymbol{K}_{\varphi_{b} u_{c}}-\boldsymbol{K}_{\varphi_{b} u_{i}} \boldsymbol{K}_{u_{i} u_{i}}^{-1} \boldsymbol{K}_{u_{i} u_{c}}\right) \delta \boldsymbol{u}_{c}+\left(\boldsymbol{K}_{\varphi_{b} \varphi_{b}}-\boldsymbol{K}_{\varphi_{b} u_{i}} \boldsymbol{K}_{u_{i} u_{i}}^{-1} \boldsymbol{K}_{u_{i} \varphi_{b}}\right) \delta \boldsymbol{\varphi}_{b}=\boldsymbol{C}_{\varphi_{b}}^{\mathrm{T}} \delta \boldsymbol{\lambda}_{\varphi_{b}} \\
\left(\boldsymbol{K}_{u_{c} u_{c}}-\boldsymbol{K}_{u_{c} u_{i}} \boldsymbol{K}_{u_{i} u_{i}}^{-1} \boldsymbol{K}_{u_{i} u_{c}}\right) \delta \boldsymbol{u}_{c}+\left(\boldsymbol{K}_{u_{c} \varphi_{b}}-\boldsymbol{K}_{u_{c} u_{i}} \boldsymbol{K}_{u_{i} u_{i}}^{-1} \boldsymbol{K}_{u_{i} \varphi_{b}}\right) \delta \boldsymbol{\varphi}_{b}=\delta \boldsymbol{\lambda}_{u_{c}}+\boldsymbol{C}_{\varphi_{c}}^{\mathrm{T}} \delta \boldsymbol{\lambda}_{\varphi_{b}}
\end{gathered}
$$

This system can be written in the matrix form

$$
\tilde{\boldsymbol{K}}\left[\begin{array}{c}
\delta \boldsymbol{u}_{c} \\
\delta \boldsymbol{\varphi}_{b}
\end{array}\right]=\left[\begin{array}{cc}
\tilde{\boldsymbol{K}}_{u_{c} u_{c}} & \tilde{\boldsymbol{K}}_{u_{c} \varphi_{b}} \\
\tilde{\boldsymbol{K}}_{\varphi_{b} u_{c}} & \tilde{\boldsymbol{K}}_{\varphi_{b} \varphi_{b}}
\end{array}\right]\left[\begin{array}{c}
\delta \boldsymbol{u}_{c} \\
\delta \boldsymbol{\varphi}_{b}
\end{array}\right]=\left[\begin{array}{cc}
\boldsymbol{I}_{N_{u_{c}} \times N_{u_{c}}} & \boldsymbol{C}_{\varphi_{c}}^{\mathrm{T}} \\
\mathbf{0}_{N_{\varphi_{b}} \times N_{u_{c}}} & \boldsymbol{C}_{\varphi_{b}}^{\mathrm{T}}
\end{array}\right]\left[\begin{array}{c}
\delta \boldsymbol{\lambda}_{u_{c}} \\
\delta \boldsymbol{\lambda}_{\varphi_{b}}
\end{array}\right],
$$

and can be simplified by reducing its second equation, yielding

$$
\begin{aligned}
\delta \boldsymbol{\varphi}_{b} & =\tilde{\boldsymbol{K}}_{\varphi_{b} \varphi_{b}}^{-1} \boldsymbol{C}_{\varphi_{b}}^{\mathrm{T}} \delta \boldsymbol{\lambda}_{\varphi_{b}}-\tilde{\boldsymbol{K}}_{\varphi_{b} \varphi_{b}}^{-1} \tilde{\boldsymbol{K}}_{\varphi_{b} u_{c}} \delta \boldsymbol{u}_{c} \\
\delta \boldsymbol{\lambda}_{u_{c}} & =\tilde{\tilde{\boldsymbol{K}}}_{u_{c} u_{c}} \delta \boldsymbol{u}_{c}-\left[\boldsymbol{C}_{\varphi_{c}}^{\mathrm{T}}-\tilde{\boldsymbol{K}}_{u_{c} \varphi_{b}} \tilde{\boldsymbol{K}}_{\varphi_{b} \varphi_{b}}^{-1} \boldsymbol{C}_{\varphi_{b}}^{\mathrm{T}}\right] \delta \boldsymbol{\lambda}_{\varphi_{b}}
\end{aligned}
$$

with

$$
\tilde{\tilde{\boldsymbol{K}}}_{u_{c} u_{c}}=\tilde{\boldsymbol{K}}_{u_{c} u_{c}}-\tilde{\boldsymbol{K}}_{u_{c} \varphi_{b}} \tilde{\boldsymbol{K}}_{\varphi_{b} \varphi_{b}}^{-1} \tilde{\boldsymbol{K}}_{\varphi_{b} u_{c}}
$$

In order to extract the dependency on the kinematics constraints $\boldsymbol{K}_{\mathrm{M}}$, Eqs. (C.13-C.14) are linearized as

$$
\begin{aligned}
\delta \boldsymbol{u}_{c}=\boldsymbol{S}_{u_{c}} \delta \boldsymbol{F}_{\mathrm{M}} & =\boldsymbol{S}_{\varphi_{c}} \delta \boldsymbol{K}_{\mathrm{M}} \\
\boldsymbol{C}_{\varphi_{c}} \delta \boldsymbol{u}_{c}+\boldsymbol{C}_{\varphi_{b}} \delta \boldsymbol{\varphi}_{b} & =\boldsymbol{S}_{\varphi_{b}} \delta \boldsymbol{K}_{\mathrm{M}}
\end{aligned}
$$

Using Eq. (C.33), this last result is rewritten as

$$
\left(\boldsymbol{C}_{\varphi_{c}}-\boldsymbol{C}_{\varphi_{b}} \tilde{\boldsymbol{K}}_{\varphi_{b} \varphi_{b}}^{-1} \tilde{\boldsymbol{K}}_{\varphi_{b} u_{c}}\right) \delta \boldsymbol{u}_{c}+\boldsymbol{C}_{\varphi_{b}} \tilde{\boldsymbol{K}}_{\varphi_{b} \varphi_{b}}^{-1} \boldsymbol{C}_{\varphi_{b}}^{\mathrm{T}} \delta \boldsymbol{\lambda}_{\varphi_{b}}=\boldsymbol{S}_{\varphi_{b}} \delta \boldsymbol{K}_{\mathrm{M}}
$$

which becomes with the help of Eq. (C.36)

$$
\boldsymbol{C}_{\varphi_{b}} \tilde{\boldsymbol{K}}_{\varphi_{b} \varphi_{b}}^{-1} \boldsymbol{C}_{\varphi_{b}}^{\mathrm{T}} \delta \boldsymbol{\lambda}_{\varphi_{b}}=\boldsymbol{S}_{\varphi_{b}} \delta \boldsymbol{K}_{\mathrm{M}}-\left(\boldsymbol{C}_{\varphi_{c}}-\boldsymbol{C}_{\varphi_{b}} \tilde{\boldsymbol{K}}_{\varphi_{b} \varphi_{b}}^{-1} \tilde{\boldsymbol{K}}_{\varphi_{b} u_{c}}\right) \boldsymbol{S}_{\varphi_{c}} \delta \boldsymbol{K}_{\mathrm{M}}
$$

or again, defining

$$
\begin{aligned}
& \tilde{\tilde{\boldsymbol{S}}}_{\varphi_{b} \varphi_{b}}=-\boldsymbol{C}_{\varphi_{b}} \tilde{\boldsymbol{K}}_{\varphi_{b} \varphi_{b}}^{-1} \boldsymbol{C}_{\varphi_{b}}^{\mathrm{T}} \text {, and } \\
& \tilde{\tilde{\boldsymbol{S}}}_{\varphi_{b} \varphi_{c}}=\boldsymbol{S}_{\varphi_{b}}-\left(\boldsymbol{C}_{\varphi_{c}}-\boldsymbol{C}_{\varphi_{b}} \tilde{\boldsymbol{K}}_{\varphi_{b} \varphi_{b}}^{-1} \tilde{\boldsymbol{K}}_{\varphi_{b} u_{c}}\right) \boldsymbol{S}_{\varphi_{c}}
\end{aligned}
$$

which becomes

$$
\delta \boldsymbol{\lambda}_{\varphi_{b}}=-\tilde{\tilde{\boldsymbol{S}}}_{\varphi_{b} \varphi_{b}}^{-1} \tilde{\tilde{\boldsymbol{S}}}_{\varphi_{b} \varphi_{c}} \delta \boldsymbol{K}_{\mathrm{M}}
$$

Finally using this last result and Eq. (C.36) in Eq. (C.34) leads to

$$
\delta \boldsymbol{\lambda}_{u_{c}}=\left[\tilde{\tilde{\boldsymbol{K}}}_{u_{c} u_{c}} \boldsymbol{S}_{\varphi_{c}}+\left[\boldsymbol{C}_{\varphi_{c}}^{\mathrm{T}}-\tilde{\boldsymbol{K}}_{u_{c} \varphi_{b}} \tilde{\boldsymbol{K}}_{\varphi_{b} \varphi_{b}}^{-1} \boldsymbol{C}_{\varphi_{b}}^{\mathrm{T}}\right] \tilde{\tilde{\boldsymbol{S}}}_{\varphi_{b} \varphi_{b}}^{-1} \tilde{\tilde{\boldsymbol{S}}}_{\varphi_{b} \varphi_{c}}\right] \delta \boldsymbol{K}_{\mathrm{M}}
$$


The variation of the homogenized stress (C.24) yields the apparent elasticity tensor $\boldsymbol{C}_{\mathrm{M}}=\frac{\partial \boldsymbol{\Sigma}_{\mathrm{M}}}{\partial \boldsymbol{F}_{\mathrm{M}}}$ in the matrix form following

$$
\begin{aligned}
\boldsymbol{C}_{\mathrm{M}} & =\frac{1}{V(\omega)}\left[\begin{array}{lll}
\boldsymbol{I}_{9 \times 9} & \mathbf{0}_{9 \times 3} & \mathbf{0}_{9 \times 1}
\end{array}\right]\left\{\boldsymbol{S}_{\varphi_{c}}^{\mathrm{T}} \frac{\partial \boldsymbol{\lambda}_{u_{c}}}{\partial \boldsymbol{K}_{\mathrm{M}}}+\boldsymbol{S}_{\varphi_{b}}^{\mathrm{T}} \frac{\partial \boldsymbol{\lambda}_{\varphi_{b}}}{\partial \boldsymbol{K}_{\mathrm{M}}}\right\}\left[\begin{array}{c}
\boldsymbol{I}_{9 \times 9} \\
\mathbf{0}_{3 \times 9} \\
\mathbf{0}_{1 \times 9}
\end{array}\right] \\
& =\frac{1}{V(\omega)}\left[\begin{array}{lll}
\boldsymbol{I}_{9 \times 9} & \mathbf{0}_{9 \times 3} & \mathbf{0}_{9 \times 1}
\end{array}\right]\left\{\tilde{\tilde{\boldsymbol{S}}}_{\varphi_{c} \varphi_{c}}-\tilde{\tilde{\boldsymbol{S}}}_{\varphi_{c} \varphi_{b}} \tilde{\tilde{\boldsymbol{S}}}_{\varphi_{b} \varphi_{b}}^{-1} \tilde{\tilde{\boldsymbol{S}}}_{\varphi_{b} \varphi_{c}}\right\}\left[\begin{array}{c}
\boldsymbol{I}_{9 \times 9} \\
\mathbf{0}_{3 \times 9} \\
\mathbf{0}_{1 \times 9}
\end{array}\right],
\end{aligned}
$$

with

$$
\begin{aligned}
\tilde{\tilde{\boldsymbol{S}}}_{\varphi_{c} \varphi_{c}} & =\boldsymbol{S}_{\varphi_{c}}^{\mathrm{T}} \tilde{\tilde{\boldsymbol{K}}}_{u_{c} u_{c}} \boldsymbol{S}_{\varphi_{c}} \text {, and } \\
\tilde{\tilde{\boldsymbol{S}}}_{\varphi_{c} \varphi_{b}} & =\boldsymbol{S}_{\varphi_{b}}^{\mathrm{T}}-\boldsymbol{S}_{\varphi_{c}}^{\mathrm{T}}\left[\boldsymbol{C}_{\varphi_{c}}^{\mathrm{T}}-\tilde{\boldsymbol{K}}_{u_{c} \varphi_{b}} \tilde{\boldsymbol{K}}_{\varphi_{b} \varphi_{b}}^{-1} \boldsymbol{C}_{\varphi_{b}}^{\mathrm{T}}\right] .
\end{aligned}
$$

The homogenized conductivity tensor $\boldsymbol{\kappa}_{\mathrm{M}}=-\frac{\partial \boldsymbol{q}_{\mathrm{M}}}{\partial \nabla_{M} \vartheta_{\mathrm{M}}}$ is directly obtained from Eq. (C.25) as

$$
\boldsymbol{\kappa}_{\mathrm{M}}=-\frac{1}{V(\omega)}\left[\begin{array}{ccc}
\mathbf{0}_{3 \times 9} & \boldsymbol{I}_{3 \times 3} & \mathbf{0}_{3 \times 1}
\end{array}\right]\left\{\tilde{\tilde{\boldsymbol{S}}}_{\varphi_{c} \varphi_{c}}-\tilde{\tilde{\boldsymbol{S}}}_{\varphi_{c} \varphi_{b}} \tilde{\tilde{\boldsymbol{S}}}_{\varphi_{b} \varphi_{b}}^{-1} \tilde{\tilde{\boldsymbol{S}}}_{\varphi_{b} \varphi_{c}}\right\}\left[\begin{array}{c}
\mathbf{0}_{9 \times 3} \\
\boldsymbol{I}_{3 \times 3} \\
\mathbf{0}_{1 \times 3}
\end{array}\right] .
$$

Finally, the apparent thermal expansion tensor $\boldsymbol{\alpha}_{\mathrm{M}}$ can be extracted in the vector form using $-\boldsymbol{C}_{\mathrm{M}} \boldsymbol{A}_{\mathrm{M}}=$ $\frac{\partial \boldsymbol{\Sigma}_{\mathrm{M}}}{\partial \vartheta_{\mathrm{M}}}$. Using the variation of the homogenized stress (C.24) yields

$$
-\boldsymbol{C}_{\mathrm{M}} \boldsymbol{A}_{\mathrm{M}}=\frac{1}{V(\omega)}\left[\begin{array}{ccc}
\boldsymbol{I}_{9 \times 9} & \mathbf{0}_{9 \times 3} & \mathbf{0}_{9 \times 1}
\end{array}\right]\left\{\tilde{\tilde{\boldsymbol{S}}}_{\varphi_{c} \varphi_{c}}-\tilde{\tilde{\boldsymbol{S}}}_{\varphi_{c} \varphi_{b}} \tilde{\tilde{\boldsymbol{S}}}_{\varphi_{b} \varphi_{b}}^{-1} \tilde{\tilde{\boldsymbol{S}}}_{\varphi_{b} \varphi_{c}}\right\}\left[\begin{array}{c}
\mathbf{0}_{9 \times 1} \\
\mathbf{0}_{3 \times 1} \\
\boldsymbol{I}_{1 \times 1}
\end{array}\right] .
$$

\section{Appendix D. Generation of the non-Gaussian random vector field $\mathcal{V}^{\prime}(x, \boldsymbol{\theta})$}

This section details the generation of a non-Gaussian random vector field using the spectral representation method developed in $[45,34,5]$, which is applied to generate the required random field $\mathcal{V}^{\prime}(\boldsymbol{x}, \boldsymbol{\theta})$, see Section 3.2.2. We assume in this paper that the random field $\mathcal{V}^{\prime}(\boldsymbol{x}, \boldsymbol{\theta})$ is a zero-mean homogeneous field. Its (normalized) cross-correlation matrix $\boldsymbol{R}_{\mathcal{V}^{\prime}}(\boldsymbol{\tau})$ (68) has been obtained through Eqs. (69), (70), (71), and (72), the definition of $\mathcal{V}^{\prime}$, and using the samples of the SVE elasticity tensors and thermal conductivity tensors. Since the homogenized thermal conductivity tensors have only three non-zero entries, together with the 21 independent entries of the elastic tensors, the vector $\mathcal{V}^{\prime}$ has 24 entries, and is written as $\left[\mathcal{V}^{(1)}(\boldsymbol{x}), \mathcal{V}^{\prime(2)}(\boldsymbol{x}), \ldots, \mathcal{V}^{(24)}(\boldsymbol{x})\right]$. Its auto/cross-correlation matrix $\boldsymbol{R}_{\mathcal{V}^{\prime}}(\boldsymbol{\tau})$ has thus a size of $24 \times 24$ entries $R_{\mathcal{V}^{\prime}}^{(r s)}(\boldsymbol{\tau}), r, s=1,2, \ldots, 24$. 
In order to use the Discrete Fourier Transform (DFT), the auto/cross-correlation $\boldsymbol{R}_{\mathcal{V}^{\prime}}(\boldsymbol{\tau})$ is computed on evenly spaced discrete points for a given distance at which it has already reached zero. Then $\boldsymbol{R}_{\mathcal{V}^{\prime}}(\boldsymbol{\tau})$ is periodized, details can be found in [22, Appendix C], and the result is denoted by $\boldsymbol{R}_{\mathcal{V}^{\prime}}^{\mathrm{P}}(\boldsymbol{\tau})$. The target spectrum is thus evaluated by

$$
\boldsymbol{S}_{\text {Target }}^{(r s)}(\boldsymbol{\omega})=\left(\Delta \tau_{x} \Delta \tau_{y} \Delta \tau_{z}\right) \boldsymbol{S}_{\text {Target }}^{(r s)}[\boldsymbol{\omega}]=\left(\Delta \tau_{x} \Delta \tau_{y} \Delta \tau_{z}\right) \sum_{\boldsymbol{\tau}=\mathbf{0}}^{l} R_{\mathcal{V}^{\prime}}^{\mathrm{P}(r s)}(\boldsymbol{\tau}) e^{-2 \pi i \boldsymbol{\tau} \cdot \boldsymbol{\omega}},
$$

where $\boldsymbol{S}_{\text {Target }}^{(r s)}(\boldsymbol{\omega})$ is the continuous form of the spectrum and $\boldsymbol{S}_{\text {Target }}^{(r s)}[\boldsymbol{\omega}]$ is its discrete form, $\boldsymbol{\tau}=\left[\tau_{x}, \tau_{y}, \tau_{z}\right]$ and $\boldsymbol{l}=\left[l_{x}, l_{y}, l_{z}\right]$ are respectively the coordinates of the discrete points and the size of sampling field of $\boldsymbol{R}_{\mathcal{V}^{\prime}}^{\mathrm{P}}(\boldsymbol{\tau})$ in the $3 \mathrm{D}$ case, and where $\left[\Delta \tau_{x}, \Delta \tau_{y}, \Delta \tau_{z}\right]$ is the coordinates increment.

The non-Gaussian vector field can now be generated following the steps:

Step 1. Set the random variables in the spectral representation formula for the generation of Gaussian field, and set $\boldsymbol{S}_{\mathrm{G}, 0}^{(r s)}(\boldsymbol{\omega})=\boldsymbol{S}_{\text {Target }}^{(r s)}(\boldsymbol{\omega})$; Iteration index is initiated at $i=0$;

Step 2. Generate the Gaussian field $\mathcal{V}_{\mathrm{G}}^{\prime}(\boldsymbol{x})$ with $\boldsymbol{S}_{\mathrm{G}, i}^{(r s)}(\boldsymbol{\omega})$, following the process detailed in [22, Appendix C];

Step 3. Compute the variance $\sigma_{\mathcal{V}_{\mathrm{G}}^{\prime(r)}}$ of each component $\mathcal{V}_{\mathrm{G}}^{\prime(r)}(\boldsymbol{x})$, and map the Gaussian field to a non-Gaussian field by [5]

$$
\mathcal{V}_{\mathrm{NG}}^{\prime(r)}(\boldsymbol{x})=F_{\mathcal{V}^{\prime(r)}}{ }^{-1}\left[F_{\mathcal{V}_{\mathrm{G}}^{\prime(r)}}\left(\mathcal{V}_{\mathrm{G}}^{\prime(r)}(\boldsymbol{x})\right)\right],
$$

where $F_{\mathcal{V}_{\mathrm{G}}^{\prime(r)}}(*)$ is a Gaussian distribution function with zero mean and variance $\sigma_{\mathcal{V}_{\mathrm{G}}^{\prime(r)}}$, and $F_{\mathcal{V}^{\prime}(r)}^{-1}$ is the inverse of the marginal distribution function of random variable $\mathcal{V}^{\prime(r)}$, which is obtained from the micro-sampling of SVEs;

Step 4. Compute the Fourier transform of $\mathcal{V}_{\mathrm{NG}}^{(r)}(\boldsymbol{x})$ by

$$
\hat{\mathcal{V}}_{\mathrm{NG}}^{(r)}(\boldsymbol{\omega})=\sum_{\boldsymbol{x}=\mathbf{0}}^{\boldsymbol{l}} \mathcal{V}_{\mathrm{NG}}^{\prime(r)}(\boldsymbol{x}) e^{-2 \pi i \boldsymbol{x} \cdot \boldsymbol{\omega}} ;
$$

and evaluate the spectrum of $\mathcal{V}_{\mathrm{NG}}^{\prime}(\boldsymbol{x})$ by

$$
\boldsymbol{S}_{\mathrm{NG}}^{(r s)}(\boldsymbol{\omega})=\frac{1}{N} \overline{\hat{\mathcal{V}}}_{\mathrm{NG}}^{\prime(r)}(\boldsymbol{\omega}) \hat{\mathcal{V}}_{\mathrm{NG}}^{(s)}(\boldsymbol{\omega}),
$$

where $N$ is the total number of discrete points of the generated field, and $\overline{\hat{\mathcal{V}}}_{\mathrm{NG}}^{\prime(r)}(\boldsymbol{\omega})$ is the conjugate of $\hat{\mathcal{V}}_{\mathrm{NG}}^{(r)}(\boldsymbol{\omega})$;

Step 5. Compare $\boldsymbol{S}_{\mathrm{NG}}^{(r s)}(\boldsymbol{\omega})$ with $\boldsymbol{S}_{\text {Target }}^{(r s)}(\boldsymbol{\omega})$, the error being calculated by [34]

$$
e r r=\frac{\int_{\Omega_{\omega}}\left|\boldsymbol{S}_{\mathrm{NG}}^{(r r)}(\boldsymbol{\omega})-\boldsymbol{S}_{\text {Target }}^{(r r)}(\boldsymbol{\omega})\right| \mathrm{d} \boldsymbol{\omega}}{\int_{\Omega_{\omega}}\left|\boldsymbol{S}_{\text {Target }}^{(r r)}(\boldsymbol{\omega})\right| \mathrm{d} \boldsymbol{\omega}},
$$

where $\Omega_{\omega}$ refers to the frequency domain; 
Step 6. If the error is lower than the given tolerance, $\operatorname{err}<T o l$, stop the iteration and $\boldsymbol{V}_{\mathrm{NG}}^{\prime}(\boldsymbol{x})$ is the required random field; Else, if err $>$ Tol, update the spectrum by ${ }^{4}$

$$
\begin{aligned}
& \boldsymbol{S}_{\mathrm{G}, i+1}^{(r r)}(\boldsymbol{\omega})=\boldsymbol{S}_{\mathrm{G}, i}^{(r r)}(\boldsymbol{\omega}) \frac{\boldsymbol{S}_{\text {Target }}^{(r r)}(\boldsymbol{\omega})}{\boldsymbol{S}_{\mathrm{NG}}^{(r r)}(\boldsymbol{\omega})} \\
& \boldsymbol{S}_{\mathrm{G}, i+1}^{(r s)}(\boldsymbol{\omega})=\boldsymbol{S}_{\mathrm{G}, i}^{(r s)}(\boldsymbol{\omega})\left(\frac{\boldsymbol{S}_{\text {Target }}^{(r r)}(\boldsymbol{\omega}) \boldsymbol{S}_{\text {Target }}^{(s s)}(\boldsymbol{\omega})}{\boldsymbol{S}_{\mathrm{NG}}^{(r r)}(\boldsymbol{\omega}) \boldsymbol{S}_{\mathrm{NG}}^{(s s)}(\boldsymbol{\omega})}\right)^{\frac{1}{2}} ;
\end{aligned}
$$

set $i=i+1 ;$ and go back to step 2 .

In our application, because the sample points of each random field are not enough to calculate the converged spectrum $\boldsymbol{S}_{\mathrm{NG}}^{(r s)}(\boldsymbol{\omega})$, thousands of random fields are generated at once to calculate the converged spectrum $\boldsymbol{S}_{\mathrm{NG}}^{(r s)}(\boldsymbol{\omega})$. After iterations, the final spectrum $\boldsymbol{S}_{\mathrm{G}}^{(r s)}(\boldsymbol{\omega})$ is obtained. In the real applications, the obtained final spectrum $\boldsymbol{S}_{\mathrm{G}}^{(r s)}(\boldsymbol{\omega})$ is used to generate the Gaussian random vector fields which will be mapped to the required non-Gaussian vector field following the process described in Step 3.

Since the final spectrum is given in its continuous form, we can decrease the frequency increments, such as setting $\Delta \omega_{i}=1 / 2 l_{i},(i=x, y, z)$, in the spectral domain when a large random field $\boldsymbol{l}$ is required. We need to note that the obtained spectrum corresponds to periodical auto/cross-correlation function $\boldsymbol{R}_{\mathcal{V}^{\prime}}^{\mathrm{P}}(\boldsymbol{\tau})$, therefore, only half the size of the generated field can be used. More details on this point can be found in [22, Appendix C].

\section{Acknowledgment}

The research has been funded by the Walloon Region under the agreement no 1117477 (CT-INT 201111-14) in the context of the ERA-NET MNT framework.

\section{References}

[1] Ainsworth, M., 2001. Essential boundary conditions and multi-point constraints in finite element analysis. Computer Methods in Applied Mechanics and Engineering 190 (48), 6323 - 6339.

URL http://www.sciencedirect.com/science/article/pii/S0045782501002365

[2] Asheghi, M., Leung, Y. K., Wong, S. S., Goodson, K. E., 1997. Phonon-boundary scattering in thin silicon layers. Applied Physics Letters 71 (13), 1798-1800.

URL http://scitation.aip.org/content/aip/journal/apl/71/13/10.1063/1.119402

[3] Baxter, S., Graham, L., 2000. Characterization of random composites using moving-window technique. Journal of Engineering Mechanics 126 (4), 389-397.

[4] Clément, A., Soize, C., Yvonnet, J., 2012. Computational nonlinear stochastic homogenization using a nonconcurrent multiscale approach for hyperelastic heterogeneous microstructures analysis. International Journal for Numerical Methods in Engineering 91 (8), 799-824.

\footnotetext{
${ }^{4}$ The update step is modified compared to the reference [34] to achieve a better convergence
} 
[5] Deodatis, G., Micaletti, R., 2001. Simulation of highly skewed non-gaussian stochastic processes. Journal of Engineering Mechanics 127 (12), 1284-1295.

[6] Der Kiureghian, A., Ke, J., 1988. The stochastic finite element method in structural reliability. Probabilistic Engineering Mechanics $3(2), 83-91$.

[7] Devanarayanan, S., Krishnan, R. S., Srinivasan, R., 1979. Thermal expansion of crystals. In: International Series in the Science of the Solid State. Vol. 12 of International Series in the Science of the Solid State. Pergamon, pp. ii -. URL http://www.sciencedirect.com/science/article/pii/B9780080214054500019

[8] Duwel, A., Gorman, J., Weinstein, M., Borenstein, J., Ward, P., 2003. Experimental study of thermoelastic damping in MEMS gyros. Sensors and Actuators A: Physical 103 (12), 70 - 75, micromechanics section of Sensors and Actuators, based on contributions revised from the Technical Digest of the 15th IEEE International conference on Micro Electro mechanical Systems (MEMS 2002).

URL http://www.sciencedirect.com/science/article/pii/S0924424702003187

[9] Geers, M., Kouznetsova, V., Brekelmans, W., 2010. Multi-scale computational homogenization: Trends and challenges. Journal of Computational and Applied Mathematics 234 (7), $2175-2182$.

[10] Gusella, V., Cluni, F., 2006. Random field and homogenization for masonry with nonperiodic microstructure. Journal of Mechanics of Materials and Structures 1 (2), 357-386.

[11] Harada, T., Shinozuka, M., 1986. The scale of correlation for stochastic fields-technical report. Department of Civil Engineering and Engineering Mechanics, Columbia University, New York, NY.

[12] Hill, R., 1963. Elastic properties of reinforced solids: Some theoretical principles. Journal of the Mechanics and Physics of Solids $11(5), 357-372$.

[13] Hoang, T., Guerich, M., Yvonnet, J., 2015. Determining the size of RVE for nonlinear random composites in an incremental computational homogenization framework. Journal of Engineering Mechanics, Accepted.

URL https://hal-upec-upem.archives-ouvertes.fr/hal-01228966

[14] Hopcroft, M., Nix, W., Kenny, T., April 2010. What is the young's modulus of silicon? Microelectromechanical Systems, Journal of 19 (2), 229-238.

[15] Houston, B., Photiadis, D., Vignola, J., Marcus, M., Liu, X., Czaplewski, D., Sekaric, L., Butler, J., Pehrsson, P., Bucaro, J., 2004. Loss due to transverse thermoelastic currents in microscale resonators. Materials Science and Engineering: A 370 (12), 407 - 411, 13th International Conference on Internal Friction and Ultrasonic Attenuation in Solids.

URL http://www.sciencedirect.com/science/article/pii/S0921509303009055

[16] Kanit, T., Forest, S., Galliet, I., Mounoury, V., Jeulin, D., 2003. Determination of the size of the representative volume element for random composites: statistical and numerical approach. International Journal of Solids and Structures 40 (1314), 3647-3679.

[17] Kouznetsova, V., Brekelmans, W. A. M., Baaijens, F. P. T., 2001. An approach to micro-macro modeling of heterogeneous materials. Computational Mechanics 27 (1), 37-48.

[18] Lanczos, C., 1950. Dan iteration method for the solution of the eigenvalue problem of linear differential and integral operators. Journal of Research of the National Bureau of Standards 45 (4), 255.

[19] Larsson, F., Runesson, K., Saroukhani, S., Vafadari, R., 2011. Computational homogenization based on a weak format of micro-periodicity for rve-problems. Computer Methods in Applied Mechanics and Engineering 200 (1-4), 11-26. URL http://www.sciencedirect.com/science/article/B6V29-50J9GSY-1/2/50ad840717a7cc397ab171da344df990

[20] Lepage, S., 2007. Stochastic finite element method for the modeling of thermoelastic damping in micro-resonators. Leloup.

[21] Lifshitz, R., Roukes, M. L., Feb 2000. Thermoelastic damping in micro- and nanomechanical systems. Phys. Rev. B 61, 5600-5609.

URL http://link.aps.org/doi/10.1103/PhysRevB.61.5600 
[22] Lucas, V., Golinval, J.-C., Paquay, S., Nguyen, V.-D., Noels, L., Wu, L., 2015. A stochastic computational multiscale approach; application to MEMS resonators. Computer Methods in Applied Mechanics and Engineering 294, 141 - 167. URL http://www.sciencedirect.com/science/article/pii/S0045782515001929

[23] Lucas, V., Golinval, J.-C., Voicu, R., Danila, M., Müller, R., Danila, M., Dinescu, A., Noels, L., Wu, L., Submitted. Propagation of material and surface profile uncertainties on MEMS micro-resonators using a stochastic second-order computational multi-scale 3 approach. Computational Materials Science.

[24] Ma, J., Sahraee, S., Wriggers, P., De Lorenzis, L., May 2015. Stochastic multiscale homogenization analysis of heterogeneous materials under finite deformations with full uncertainty in the microstructure. Comput. Mech. 55 (5), 819-835. URL http://dx.doi.org/10.1007/s00466-015-1136-3

[25] Mariani, S., Martini, R., Ghisi, A., Corigliano, A., Beghi, M., 2011. Overall elastic properties of polysilicon films: a statistical investigation of the effects of polycrystal morphology. International Journal for Multiscale Computational Engineering 9 (3), 327-346.

[26] Mariani, S., Martini, R., Ghisi, A., Corigliano, A., Simoni, B., 2011. Monte carlo simulation of micro-cracking in polysilicon MEMS exposed to shocks. International Journal of Fracture 167 (1), 83-101.

URL http://dx.doi.org/10.1007/s10704-010-9531-4

[27] Matthies, H. G., Brenner, C. E., Bucher, C. G., Guedes Soares, C., 1997. Uncertainties in probabilistic numerical analysis of structures and solids-stochastic finite elements. Structural Safety 19 (3), 283 - 336, devoted to the work of the Joint Committee on Structural Safety.

[28] Maugin, G. A., 1999. The Thermomechanics of Nonlinear Irreversible Behaviors. Vol. 27 of World Scientific Series on Nonlinear Science Series A. World Scientific.

URL http://www. worldscientific. com/worldscibooks/10.1142/3700

[29] McConnell, A., Uma, S., Goodson, K. E., Sep 2001. Thermal conductivity of doped polysilicon layers. Microelectromechanical Systems, Journal of 10 (3), 360-369.

[30] Miehe, C., Koch, A., 2002. Computational micro-to-macro transitions of discretized microstructures undergoing small strains. Archive of Applied Mechanics 72 (4-5), 300-317.

[31] Nguyen, V.-D., Béchet, E., Geuzaine, C., Noels, L., 2012. Imposing periodic boundary condition on arbitrary meshes by polynomial interpolation. Computational Materials Science 55, 390-406.

[32] Ostoja-Starzewski, M., Wang, X., 1999. Stochastic finite elements as a bridge between random material microstructure and global response. Computer Methods in Applied Mechanics and Engineering 168 (14), 35 - 49.

[33] Özdemir, I., Brekelmans, W. A. M., Geers, M. G. D., 2008. Computational homogenization for heat conduction in heterogeneous solids. International Journal for Numerical Methods in Engineering 73 (2), 185-204.

URL http://dx.doi.org/10.1002/nme.2068

[34] Popescu, R., Deodatis, G., Prevost, J., 1998. Simulation of homogeneous nongaussian stochastic vector fields. Probabilistic Engineering Mechanics 13 (1), $1-13$.

URL http://www.sciencedirect.com/science/article/pii/S0266892097000015

[35] Serra, E., Bonaldi, M., 2009. A finite element formulation for thermoelastic damping analysis. International Journal for Numerical Methods in Engineering 78 (6), 671-691.

URL http://dx.doi.org/10.1002/nme.2502

[36] Shinokuza, M., Astill, C. J., Apr 1972. Random eigenvalue problems in structural analysis. AIAA Journal 10 (4), $456-462$.

[37] Shinozuka, M., Deodatis, G., 1988. Response variability of stochastic finite element systems. Journal of Engineering Mechanics 114 (3), 499-519.

[38] Shinozuka, M., Jan, C.-M., 1972. Digital simulation of random processes and its applications. Journal of Sound and Vibration 25 (1), 111 - 128. 
[39] Stefanou, G., Savvas, D., Papadrakakis, M., 2015. Stochastic finite element analysis of composite structures based on material microstructure. Composite Structures 132, $384-392$.

URL http://www.sciencedirect.com/science/article/pii/S0263822315004183

[40] Temizer, I., Wriggers, P., 2011. Homogenization in finite thermoelasticity. Journal of the Mechanics and Physics of Solids $59(2), 344-372$.

URL http://www.sciencedirect.com/science/article/pii/S002250961000219X

[41] Terada, K., Hori, M., Kyoya, T., Kikuchi, N., 2000. Simulation of the multi-scale convergence in computational homogenization approaches. International Journal of Solids and Structures 37 (16), 2285-2311.

URL http://www.sciencedirect.com/science/article/B6VJS-3YDFYCH-4/2/788d085d620c6c5ae804d8c4e4f8ae9b

[42] Trovalusci, P., Ostoja-Starzewski, M., De Bellis, M. L., Murrali, A., 2015. Scale-dependent homogenization of random composites as micropolar continua. European Journal of Mechanics - A/Solids 49 (0), 396 - 407.

[43] Wright, T., 2002. The Physics and Mathematics of Adiabatic Shear Bands. Cambridge Monographs on Mechanics. Cambridge University Press.

URL https ://books.google.com/books?id=FGilEcC2tg0C

[44] Yamazaki, F., Member, A., Shinozuka, M., Dasgupta, G., 1988. Neumann expansion for stochastic finite element analysis. Journal of Engineering Mechanics 114 (8), 1335-1354.

[45] Yamazaki, F., Shinozuka, M., 1988. Digital generation of nongaussian stochastic fields. Journal of Engineering Mechanics $114(7), 1183-1197$.

[46] Yasumura, K., Stowe, T., Chow, E., Pfafman, T., Kenny, T., Stipe, B., Rugar, D., March 2000. Quality factors in micronand submicron-thick cantilevers. Microelectromechanical Systems, Journal of 9 (1), 117-125.

[47] Yin, X., Chen, W., To, A., McVeigh, C., Liu, W., 2008. Statistical volume element method for predicting microstructureconstitutive property relations. Computer Methods in Applied Mechanics and Engineering 197 (43 - 44 ), 3516 - 3529 , stochastic Modeling of Multiscale and Multiphysics Problems.

[48] Zener, C., 1948. Elasticity and Anelasticity of Metals. University of Chicago Press. 